\title{
Review of Smart Grid Standards for Testing and Certification Landscape Analysis
}

\author{
Eugene Y. Song \\ Cuong Nguyen \\ Avi Gopstein
}




\title{
Review of Smart Grid Standards for Testing and Certification Landscape Analysis
}

\author{
Eugene Y. Song \\ Cuong Nguyen \\ Avi Gopstein \\ Smart Grid and Cyber-Physical Systems Program Office \\ Engineering Laboratory
}

This publication is available free of charge from:

https://doi.org/10.6028/NIST.TN.2042

April 2019

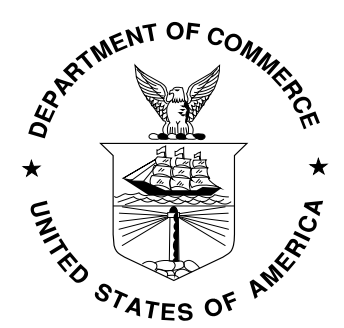

U.S. Department of Commerce Wilbur L. Ross, Jr., Secretary

National Institute of Standards and Technology Walter Copan, NIST Director and Undersecretary of Commerce for Standards and Technology 
Certain commercial entities, equipment, or materials may be identified in this document in order to describe an experimental procedure or concept adequately. Such identification is not intended to imply recommendation or endorsement by the National Institute of Standards and Technology, nor is it intended to imply that the entities, materials, or equipment are necessarily the best available for the purpose.

National Institute of Standards and Technology Technical Note 2042

Natl. Inst. Stand. Technol. Tech. Note 2042, 76 pages (April 2019) CODEN: NTNOEF

This publication is available free of charge from:

https://doi.org/10.6028/NIST.TN.2042 


\title{
Disclaimers
}

Certain commercial entities, equipment, or materials may be identified in this document to describe an experimental procedure or concept adequately. Such identification is not intended to imply recommendation or endorsement by the National Institute of Standards and Technology, nor is it intended to imply that the entities, materials, or equipment are necessarily the best available for the purpose.

\begin{abstract}
The National Institute of Standards and Technology (NIST) Smart Grid Program seeks to accelerate the development of conformity assessment or testing and certification (T\&C) programs for smart grid interoperability standards to ensure that the implementations conform to the standards' requirements and therefore enhance interoperability of smart grid devices and systems. This document presents a review of the current state of T\&C for smart grid standards using a set of metrics developed from functional aspects of the standards. These functional metrics include information model and model mapping, communication protocol and protocol mapping, device physical performance, test methodologies, guidelines and best practices, and cybersecurity. These metrics are used to analyze smart grid standards and the T\&C availability of these standards was also evaluated. The 240 smart grid standards analyzed were drawn primarily from three resources: the Smart Electric Power Alliance (SEPA) Catalog of Standards (CoS), the 2014 release of NIST’s Framework and Roadmap for Smart Grid Interoperability Standards Release 3.0, and the Union of the Electricity Industry (EURELECTRIC) and European Distribution System Operator (EDSO) priorities for smart grid standardization position paper. The results of the smart grid standards T\&C landscape analysis are provided in the report. These results indicate that only a small percentage of smart grid standards have available T\&C programs.
\end{abstract}

\section{Key words}

Conformity assessment; interoperability; metrics; smart grid; standards; testing and certification (T\&C). 


\section{Table of Contents}

1. Introduction .......................................................................................................................... 1

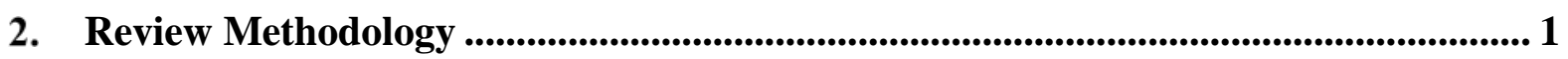

3. Identified Smart Grid Standards for Review.................................................................. 3

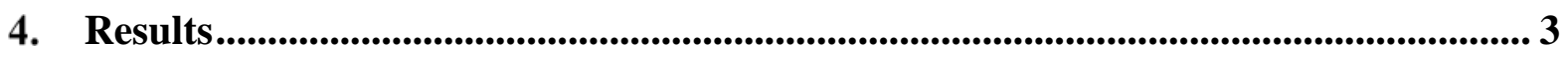

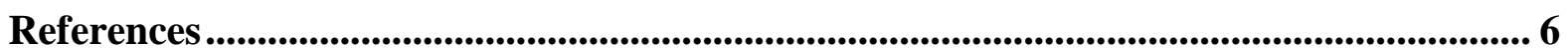

Appendix A: List of Acronyms and Abbreviations ................................................................... 7

Appendix B: A List of Reviewed Smart Grid Standards ....................................................... 11

\section{List of Tables}

Table 1- Standard Category and Number of Standards Evaluated .............................................. 4

Table 2- A List of Reviewed Smart Grid Standards ............................................................ 11

\section{List of Figures}

Figure 1. IEC 61850 Functionality and Applications. ............................................................. 2

Figure 2. Review Results of Smart Grid Standards for T\&C Landscape. .................................. 5 


\section{Introduction}

Smart grid standards play a key role in the deployment of new technologies for the power grid. Standards can encourage innovation, boost productivity, and enhance economic efficiency by reducing or eliminating technical barriers for new technologies [1], and those standards that advance interoperability help maximize operational benefits while minimizing deployment costs for new equipment [2]. Testing and certification (T\&C) programs provide a mechanism for all stakeholders to gain confidence that equipment function conforms to standards and will operate as intended, and are important to realize the benefits of a strong standards ecosystem [3].

The National Institute of Standards and Technology (NIST) previously conducted a landscape analysis study in 2010 to review the availability of T\&C for smart grid standards [4]. The study found a limited number of existing smart grid test programs. To gain an understanding of the evolving $\mathrm{T} \& \mathrm{C}$ landscape for smart grid interoperability since the initial study, NIST compiled a representative list of smart grid standards and reviewed the status of associated T\&C programs in this report. Because the complexity of smart grid standards and their applications have increased commensurately with that of the electrical grid, NIST assessed the T\&C landscape for interoperability impact that each standard — and any associated T\&C program-might have from a functional perspective.

\section{Review Methodology}

Applications for smart grid standards may span numerous dimensions of the grid. For example, the International Electrotechnical Commission (IEC) 61850 standard [5] shown in Figure 1 has applications in each of the NIST Smart Grid Conceptual Model domains [2], including generation, transmission, distribution, customer, market, operations, and service provider. It also has diverse functionality or capabilities requirements, such as for the structure of an information model, communication protocols and specifications, performance requirements, testing methods, and so on. Figure 1 also shows the IEC 61850 device structures (upper left) [7], object model (upper middle) [8], communication protocols (upper right) [9], and IEC 61850 applications and interface specifications across the grid domains (red circles in lower graphic) [6]. Compounding the complexity inherent to this breadth of application is the fact that smart grid standards-IEC 61850 included-will likely have different use cases in each domain or subdomain.

To identify those standards that might impact smart grid interoperability, NIST employed a functional classification system to complement the traditional architectural view of standards applications. Standards were functionally categorized as relating to information model and model mapping, communication protocol and protocol mapping, physical performance specification, test methodology, guideline and best practice, and cybersecurity; standards could be assigned multiple categories. Those standards associated with the following three function classes were deemed relevant to smart grid interoperability:

1) Information model and model mapping;

2) Communication protocol and protocol mapping; and

3) Physical performance specification. 


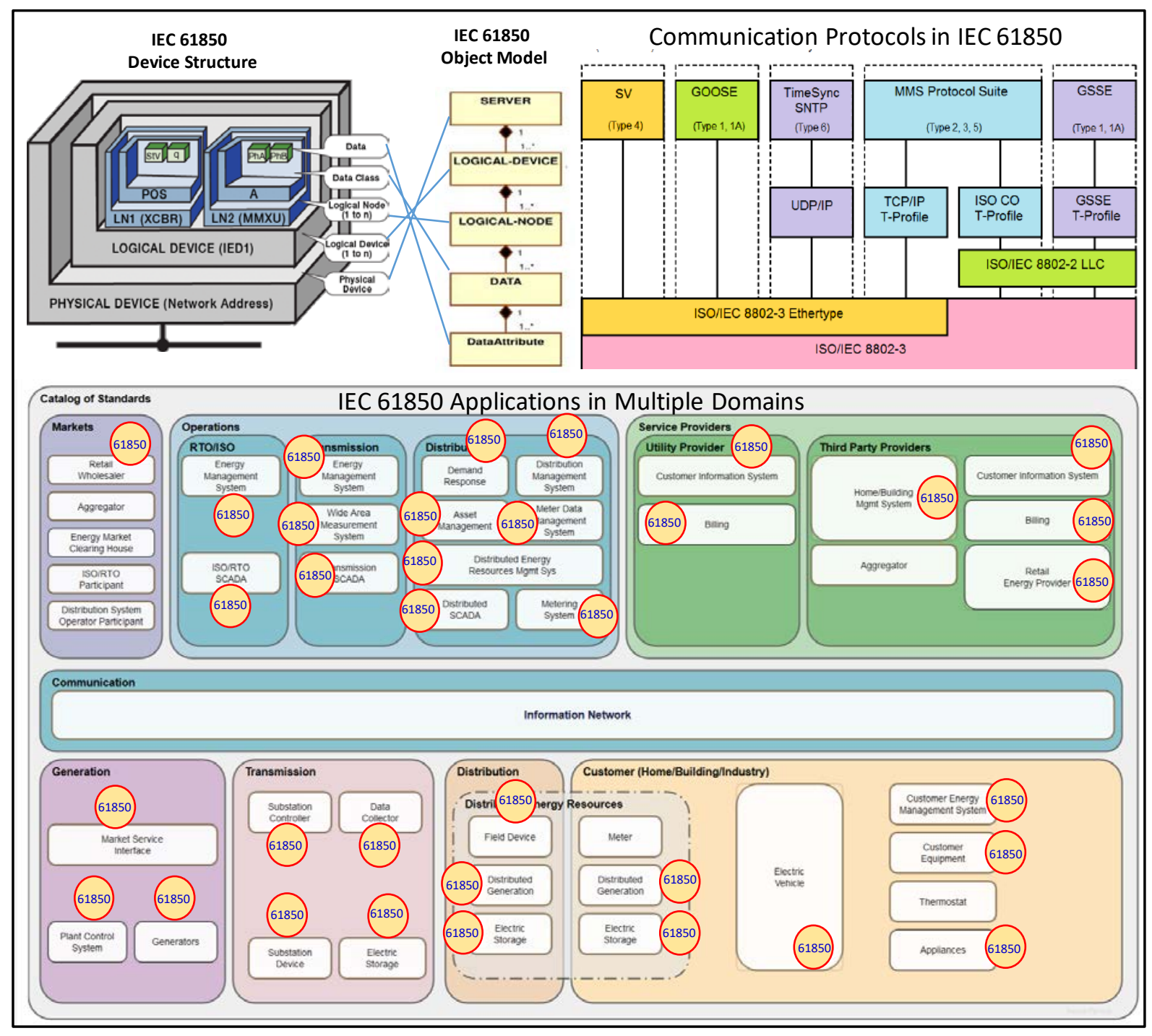

Figure 1. IEC 61850 functionality and applications in multiple domains ${ }^{1}$.

Each standard was reviewed to determine if an associated T\&C program is currently available or planned to for the future. The review covered different types of testing, including first party (self-certification), second party (user certification), and third party (independent certification) testing. NIST's determination of T\&C program availability (or planned availability) was based on publicly available documentation on testing services associated with each standard, T\&C program operators, test labs, and certification bodies. NIST did not assess the quality or effectiveness of T\&C programs in this review and makes no claim regarding the relevance or importance of any T\&C program.

\footnotetext{
${ }^{1}$ http://www.gridstandardsmap.com/. Multiple domains image used with permission from SEPA.
} 


\section{Identified Smart Grid Standards for Review}

This review included 240 individual standards from twenty-two standards development organizations covering more than thirty-four families of standards. Drawn from numerous sources including those described below, NIST makes no claim regarding the importance of any standard included in these lists, or the relevance or importance of any standard not included in these lists. Sources included:

\section{Smart Electric Power Alliance (SEPA) Catalog of Standards (CoS)}

SEPA produces and maintains the $\mathrm{CoS}$ as a reference to the electric grid community. With 81 smart grid standards, the catalog is a compendium of standards and practices considered to be relevant for the development and deployment of a robust, interoperable, and secure grid [6].

NIST Framework and Roadmap for Smart Grid Interoperability Standards, R3.0 The 2014 version of the NIST Interoperability Framework identifies 72 smart gridrelevant standards. Guiding principles for identifying standards for implementation and including them on this list are provided on pages 55-56 of this report [2].

\section{European Distribution System Operator Priority List}

The European Commission has identified broad priorities for smart grid standardization in Europe. Based on these recommendations, utility members of the Union of the Electricity Industry (EURELECTRIC) and European Distribution System Operator (EDSO) for smart grids have developed a list of priority standards and related activities for implementation in the smart grid [5] and the IEC smart grid standards map [10].

Most, but not all, of the standards used for this review were drawn from the above three sources. Yet because standards evolve in concert with technology, some standards have been updated or modified since these lists were published and so the newer versions were also reviewed. For example, the American National Standards Institute (ANSI) C12.20-2010 [11] is included in both the SEPA CoS and the NIST Framework R3.0, but a major update with significant new performance criteria in ANSI C12.20-2015 [12] has since been released and was included in this review.

A preliminary analysis was presented at the NIST Testing \& Certification Workshop on July 9, 2018 [13], and stakeholder feedback was incorporated in this report. The full list of smart grid standards and associated reviews are provided in Appendix B. Only standards published before March 2019 were included in this analysis.

\section{Results}

Of the 240 standards reviewed, 169 standards were found to be functionally related to interoperability, and of those only a small percentage were found to have T\&C programs of any form-either existing or planned. Table 1 provides an overview of the total number of standards determined to be in each functional interoperability category (rows), as well as the number and implementation structure for any associated T\&C programs; standards that 
address multiple interoperability functional categories were identified and grouped accordingly. Certification type is broken into three: independent third party certification authorities (ITCA), first party (vendor self-certification), and second party (user certification). Because there were only two well-known second party T\&C programs, these were grouped with first party programs in Table 1. The "Planned" column indicates when a T\&C program is planned but not yet available for these smart grid standards.

Table 1. Standard Category and Number of Standards Evaluated.

\begin{tabular}{|l|c|c|c|c|}
\hline \multirow{2}{*}{ Standard Category } & \multicolumn{4}{c|}{ Number of Standards Evaluated } \\
\cline { 2 - 5 } & Total No. of & \multicolumn{2}{c|}{ with T\&C Programs of type: } \\
\cline { 3 - 5 } & Standards & ITCA $^{2}$ & $1^{\text {st }}$ Party & Planned \\
\hline Information Model & 34 & 5 & 0 & 2 \\
\hline Information Model \& Communication Protocol & 28 & 7 & 4 & 1 \\
\hline Communication Protocol & 77 & 21 & 2 & 1 \\
\hline Communication Protocol \& Physical Performance & 7 & 0 & 1 & 2 \\
\hline Physical Performance & 22 & 3 & 8 & 0 \\
\hline $\begin{array}{l}\text { Information Model + Communication Protocol + } \\
\text { Physical Performance }\end{array}$ & 1 & 0 & 0 & 0 \\
\hline \multicolumn{1}{|c|}{ Total } & 169 & 36 & 15 & 6 \\
\hline
\end{tabular}

A complete list of smart grid standards reviewed for T\&C availability and functional categorization is available in Appendix B. The results of this analysis are described in Table $\mathbf{1}$ and shown in Figure 2. These information model, communication protocol, and physical performance standards in Figure 2 are presented in blue, orange, and green colors, respectively. The total 240 smart grid standards are presented in the black circle. In the legend of Figure 2, independent T\&C, $1^{\text {st }}$ party T\&C, and planed T\&C programs are represented in hatches, dots, and lines, respectively. The review results reveal several important trends about the standards evaluated for this study:

- Only $21 \%$ of 169 interoperability standards have independent T\&C programs.

- When including $1^{\text {st }}$ and $2^{\text {nd }}$ party activities, only $30 \%$ of 169 interoperability standards have $\mathrm{T} \& \mathrm{C}$ programs.

- Physical performance standards are most likely to have T\&C programs, but the substantial majority of those are not independent.

- There is no existing or planned path to device certification for the substantial majority of interoperability relevant smart grid standards.

The challenge of assuring interoperability is made more complex by the fact that interoperability for the smart grid demands more than the mere exchange of original or unprocessed data — sometimes called syntactic interoperability — and instead requires a semantic model [14] that utilizes elements from each of the above functional categories. Looked at another way, the limited extent of $\mathrm{T} \& \mathrm{C}$ programs within each functional grouping

\footnotetext{
${ }^{2}$ Independent third party certification authorities (ITCAs): https://www.iassc.org/question/why-is-an-independent-third-party-certificationimportant/
} 
means the likely availability of certification-based assurance programs becomes vanishingly small once one realizes that achieving a full measure of device or systems interoperability requires a combined set of capabilities built from standards across multiple functional groupings.

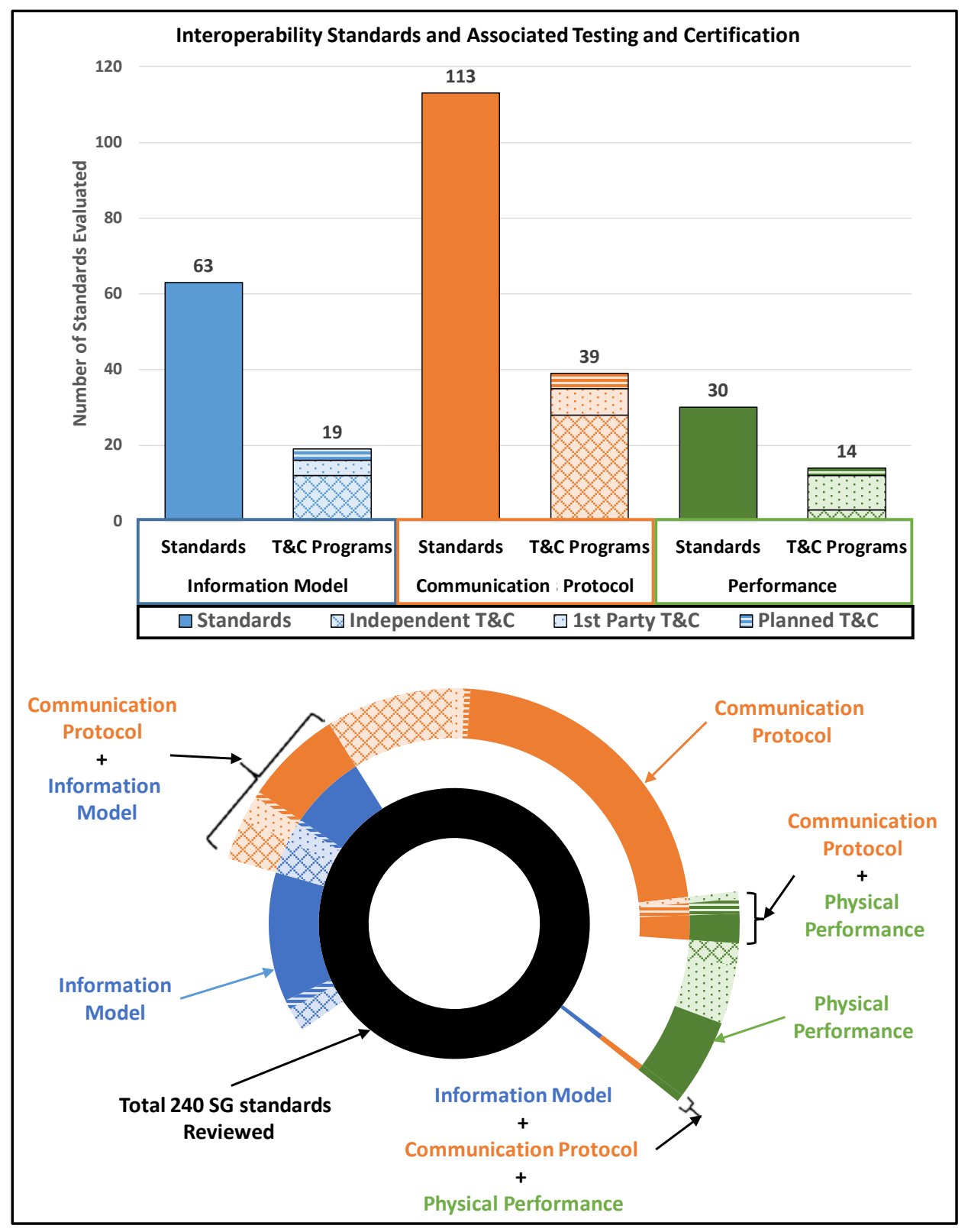

Figure 2. Review results of smart grid standards for T\&C landscape.

\section{Acknowledgments}

The authors wish to thank all those who contributed ideas and suggestions for this report. They include Dr. David Wollman and Dr. Marty Burns of EL's Smart Grid and CyberPhysical Systems Program Office, Steve Bushby of EL's Energy and Environment Division, Dr. Gerald Fitzpatrick and Thomas Nelson of PML’s Quantum Measurement Division. 


\section{References}

[1] Distribution System Operator (DSO) Priorities for Smart Grid Standardization- A EURELECTRIC / EDSO for Smart Grid joint position paper https://www.edsoforsmartgrids.eu/wp-content/uploads/public/DSO-Priorities-SmartGird-Standardisation.pdf.

[2] NIST Special Publication 1108r3, NIST Framework and Roadmap for Smart Grid Interoperability Standards, Release 3.0. https://nvlpubs.nist.gov/nistpubs/SpecialPublications/NIST.SP.1108r3.pdf.

[3] Public Utilities Commission of the State of California Resolution E-4527 (2012), http://docs.cpuc.ca.gov/PublishedDocs/Published/G000/M029/K624/29624509.PDF.

[4] Existing Conformity Assessment Program Landscape (2010), http://collaborate.nist.gov/twikisggrid/pub/SmartGrid/SGIPDocumentsAndReferencesSGTCC/D11aConformityLandscape_delivered.doc

[5] IEC 61850 Communication networks and systems for power utility automation, https://webstore.iec.ch/searchform\&q=61850.

[6] SEPA Smart Grid Catalog of Standards, https://sepapower.org/knowledge/catalog-ofstandards/catalog-of-standards-complete-list-of-entries/.

[7] Juan Gers, A PRIMER on IEC 61850 grid data communications, https://blogs.dnvgl.com/energy/a-primer-on-iec-61850-grid-data-communications.

[8] Drew Baigent, Mark Adamiak, Ralph Mackiewicz, IEC 61850 Communication Networks and Systems In Substations: An Overview for Users, https://www.slideserve.com/tate-olson/iec-61850-communication-networks-andsystems-in-substations-an-overview-for-users.

[9] IEC 61850 Protocol Libraries, http://www.xelasenergy.com/products/61850_protocols.php

[10] IEC Smart Grid Standards Map, http://smartgridstandardsmap.com/.

[11] ANSI C12.20-2010, American National Standard for Electricity Meters - 0.2 and 0.5 Accuracy Classes, https://webstore.ansi.org/Previews/PREVIEW_ANSI+C12.202010.pdf.

[12] ANSI C12.20-2015: American National Standard for Electricity Meters - 0.1, 0.2, and 0.5 Accuracy Classes, https://webstore.ansi.org/RecordDetail.aspx?sku=ANSI+C12.202015.

[13] NIST Testing \& Certification Workshop on July 9, 2018, https://www.nist.gov/sites/default/files/documents/2018/06/25/draft_tc_landscape_eval uation_final.pdf

[14] Papaioannou I., Tarantola S., Lucas A., Kotsakis E., Marinopoulos A., Ginocchi M., Olariaga Guardiola M., Masera M., Smart grid interoperability testing methodology, EUR 29416 EN, Publications Office of the European Union, Luxembourg, 2018, ISBN 978-92-79-96855-6, doi:10.2760/08049, JRC110455, http://publications.jrc.ec.europa.eu/repository/bitstream/JRC110455/kjna29416enn_fina $\underline{\text { l.pdf }}$ 


\section{Appendix A: List of Acronyms and Abbreviations}

ACSI

AES

AC

AMI

AMR

ANSI

API

ASDU

ASHRAE

BACnet

BEV

BPL

CDC

CEA

CIM

CIP

CIS

CNP

COMFEDE

COMTRADE

CoS

COSEM

CPS

CRC

CTA

DA

DC

DER

DG

DHS

DLL

DLMS/COSEM

DMS

DNP

DR

D-SCADA

DSO

DSU

ED

EDSO

EIA

EMC

EMS
Abstract Communication Service Interface

Advanced Encryption Standard

Alternating Current

Advanced Metering Infrastructure

Automated Meter Reading

American National Standards Institute

Application Programming Interface

Application Service Data Unit

American Society of Heating, Refrigerating and Air-Conditioning Engineers

Building Automation and Control Networks

Battery EV

Broadband over Power Line

Common Data Classes

Carcinoembryonic Antigen

Common Information Model

Critical Infrastructure Protection

Customer Information System

Control Network Protocol

Common Format for Event Data Exchange

Common Format for Transient Data Exchange

Catalog of Standards

Companion Specification for Energy Metering

Cyber-Physical System

Circuit Reconfiguration Controller

Consumer Technology Association

Distribution Access

Direct Current

Distributed Energy Resources

Distributed Generation

Department of Homeland Security

Data Link Layer

Device Language Message Specification/Companion Specification for Energy Metering

Distributed Management System

Distributed Network Protocol

Demand Response

Distribution SCADA

Distribution System Operators

Distribution Substation Units

End Device

European Distribution System Operator

Enzyme Immunoassay

Electromagnetic Compatibility

Energy Management System 


\begin{tabular}{|c|c|}
\hline ES & Energy System \\
\hline ESA & Electronic Sub Assembly \\
\hline ESB & Enterprise Service Bus \\
\hline ESP & Energy Service Provider \\
\hline ESPI & ESP Interface \\
\hline ESS & Energy Storage Systems \\
\hline EUI & Energy Usage Information \\
\hline $\mathrm{EV}$ & Electric Vehicle \\
\hline EVSE & EV Supply Equipment \\
\hline FP & Functional Profile \\
\hline FPI & Fault Passage Indicators \\
\hline FSGIM & Facility Smart Grid Information Model \\
\hline GOOSE & Generic Object-Oriented Substation Event \\
\hline HAN & Home Area Network \\
\hline HES & Home Electronic System \\
\hline HLDL & High-Level Data Link \\
\hline HTTP & Hyper Text Transfer Protocol \\
\hline HVAC & Heating, Ventilating, Air Conditioning \\
\hline HVAC\&R & Heating, Ventilating, Air Conditioning and Refrigerating \\
\hline ICT & Information and Communications Technology \\
\hline IEC & International Electrotechnical Commission \\
\hline IED & Intelligent Electronic Device \\
\hline IEEE & Institute of Electrical and Electronics Engineers \\
\hline IETF & Internet Engineering Task Force \\
\hline IoT & Internet of Things \\
\hline IP & Internet Protocol \\
\hline IPv6 & Internet Protocol Version 6 \\
\hline ISO & International Organization for Standardization \\
\hline IT & Information Technology \\
\hline ITU-T & International Telecommunication Union - Telecommunication \\
\hline LAN & Local Area Network \\
\hline LD & Logical Device \\
\hline $\mathrm{LN}$ & Logical Node \\
\hline LPIT & Low Power Instrument Transformers \\
\hline MAC & Media Access Control \\
\hline MCI & Modular Communications Interface \\
\hline MDM & Meter Data Management \\
\hline MDMS & Meter Data Management System \\
\hline MEMS & Microgrid Energy Management System \\
\hline MIB & Management Information Base \\
\hline MIC & Monitoring, Information exchange, and Control \\
\hline MMS & Manufacturing Message Specification \\
\hline MPC & Monitoring, Protection and Control \\
\hline MSVCB & Multicast Sampled Value Control Block \\
\hline
\end{tabular}




\begin{tabular}{|c|c|}
\hline NAESB & North American Energy Standards Board \\
\hline NB-PLC & Narrowband PLC \\
\hline NCAP & Network Capable Applications Processor \\
\hline NEMA & National Electrical Manufacturers Association \\
\hline NERC & North American Electric Reliability Corporation \\
\hline NIST & National Institute of Standards and Technology \\
\hline NN & Neighborhood Network \\
\hline NSM & Network and System Management \\
\hline OASIS & Organization for the Advancement of Structured Information Standards \\
\hline OASIS & Organization for the Advancement of Structured Information Standards \\
\hline OBIS & Object Identification System \\
\hline OFDM & Orthogonal Frequency Division Multiplexing \\
\hline OGC & Open Geospatial Consortium \\
\hline OGC-GML & Open Geospatial Consortium - Geography Markup Language \\
\hline OLE & Object Linking and Embedding \\
\hline OMS & Outage Management System \\
\hline OPC & OLE for Process Control \\
\hline OPC UA & OPC Unified Architecture \\
\hline OpenADR & Open Automated Demand Response \\
\hline OpenFMB & Open Field Message Bus \\
\hline OpenHAN & Open Home Area Network \\
\hline OSI & Open Systems Interconnection \\
\hline PAN & Personal Area Network \\
\hline PDC & Phasor Data Concentrator \\
\hline PEFC & Polymer Electrolyte Fuel Cell \\
\hline PHEV & Plug-in Hybrid Road Vehicles \\
\hline PHY & Physical Layer \\
\hline PICS & Protocol Implementation Conformance Statement \\
\hline PLC & Power Line Communication \\
\hline PMU & Phasor Measurement Unit \\
\hline PSD & Power Spectral Density \\
\hline PSTN & Public Switched Telephone Network \\
\hline PTP & Precision Time Protocol \\
\hline PV & Photovoltaic \\
\hline RBA & Role Bases Access \\
\hline RESS & Rechargeable Energy Storage Systems \\
\hline REST & Representational State Transfer \\
\hline ROCOF & Rate of Change of Frequency \\
\hline RTO & Regional Transmission Organization \\
\hline RTP & Real Time Price \\
\hline RTU & Remote Terminal Unit \\
\hline SAE & Society of Automotive Engineers \\
\hline SAS & System/Substation Automation System \\
\hline SCADA & Supervisory Control and Data Acquisition \\
\hline
\end{tabular}




\begin{tabular}{|c|c|}
\hline SCL & System/Substation Configuration Language \\
\hline SCSM & Specific Communication Service Mapping \\
\hline SEP & Smart Energy Profile \\
\hline SEPA & Smart Electric Power Alliance \\
\hline SGIP & Smart Grid Interoperability Panel \\
\hline SGIRM & Smart Grid Interoperability Reference Model \\
\hline SM & Smart Meter \\
\hline SNMP & Simple Network Management Protocol \\
\hline SNTP & Simple Network Time Protocol \\
\hline SOA & Service-oriented Architecture \\
\hline SOAP & Simple Object Access Protocol \\
\hline SPS & Special Protection Systems \\
\hline SV & Sampled Value \\
\hline $\mathrm{T} \& \mathrm{C}$ & Testing and Certification \\
\hline TASE & Telecontrol Application Service Elements \\
\hline TCP & Transmission Control Protocol \\
\hline TEDS & Transducer Electronic Data Sheet \\
\hline TIM & Transducer Interface Module \\
\hline TR & Technical Report \\
\hline TS & Technical Specification \\
\hline TSO & Transmission System Operator \\
\hline UAS & Utility Automation System \\
\hline UCA & Utility Communications Architecture \\
\hline UCAIug & UCA International Users Group \\
\hline UDP & User Datagram Protocol \\
\hline UML & Unified Model Language \\
\hline URI & Uniform Resource Identifier \\
\hline USVCB & Unicast Sampled Value Control Block \\
\hline Volt/VAR & Voltage/Volt-Ampere Reactive \\
\hline W3C & World Wide Web Consortium \\
\hline WAMPAC & Wide Area Monitoring, Protection, and Control \\
\hline WAMS & Wide Area Management Systems \\
\hline WAN & Wide Area Network \\
\hline WS & Web Service \\
\hline WSDL & Web Service Description Language \\
\hline WTIM & Wireless Transducer Interface Module \\
\hline XML & Extensible Markup Language \\
\hline XMPP & Extensible Messaging and Presence Protocol \\
\hline
\end{tabular}




\section{Appendix B: A List of Reviewed Smart Grid Standards}

T\&C Programs in Table 2 are categorized as follows: $\mathrm{x}$ - an independent T\&C authority (ITCA) exists for this standard; $\mathrm{y}-$ the ITCA program for this standard derives from requirements established in a different standard; $\mathrm{z}-1$ st or 2nd party T\&C programs exist for this standard; and p - a T\&C program is planned for this standard. This table will be separately published as a dataset and will be available in the link: https://doi.org/10.18434/M32061.

Table 2. A List of Reviewed Smart Grid Standards.

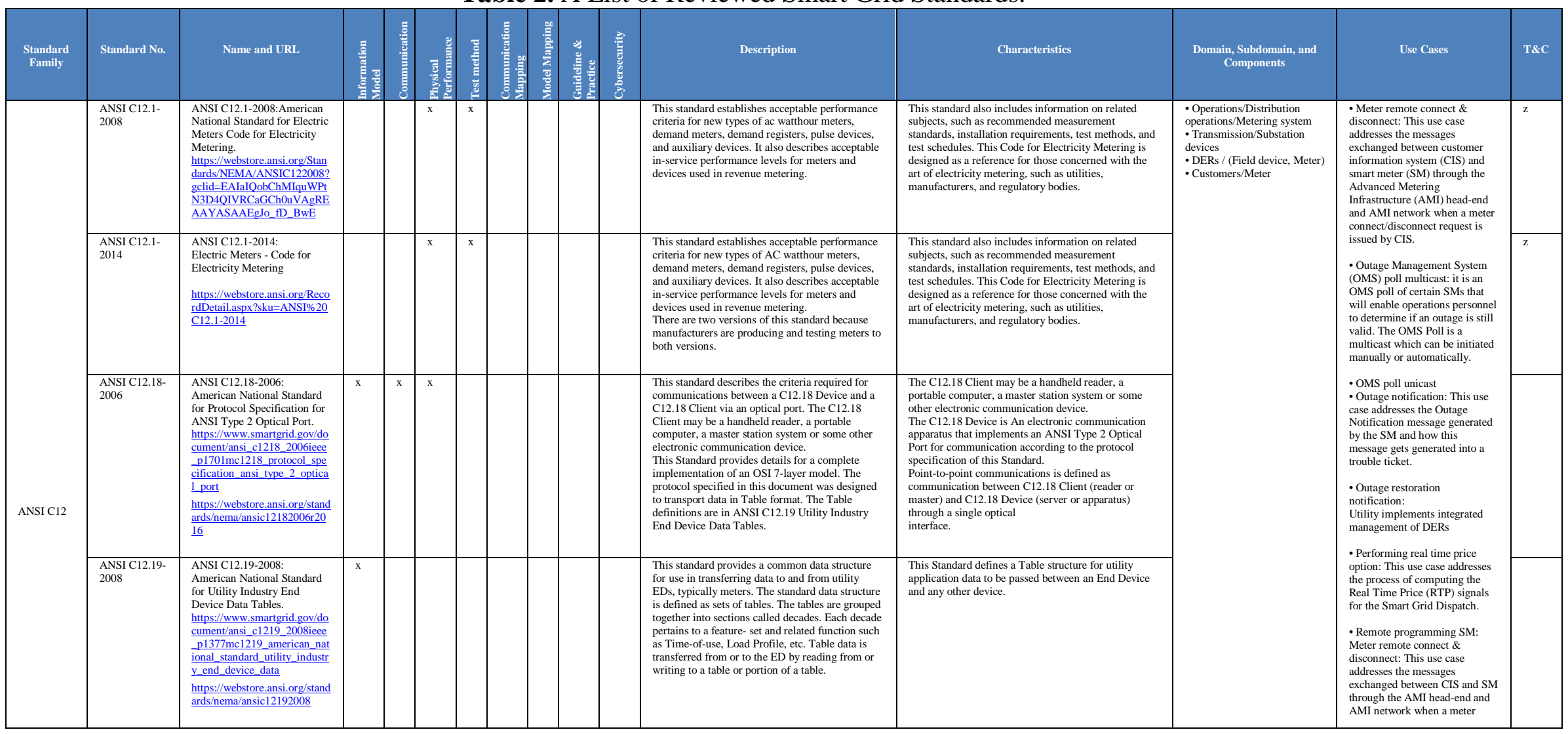




\begin{tabular}{|c|c|c|c|c|c|c|c|c|c|c|c|c|c|c|}
\hline $\begin{array}{l}\text { Standard } \\
\text { Family }\end{array}$ & Standard No. & Name and URL & & & & 尝 & & 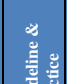 & 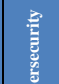 & Description & Characteristics & $\begin{array}{l}\text { Domain, Subdomain, and } \\
\text { Components }\end{array}$ & Use Cases & T\&C \\
\hline \multirow{4}{*}{ ANSI C12 } & $\begin{array}{l}{ }_{2012}^{\text {ANSI 12.19- }} \\
2012\end{array}$ & 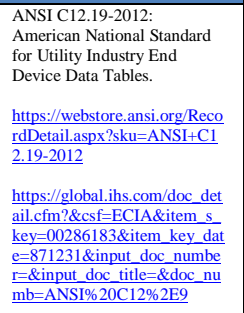 & $\mathrm{x}$ & & & & & & & 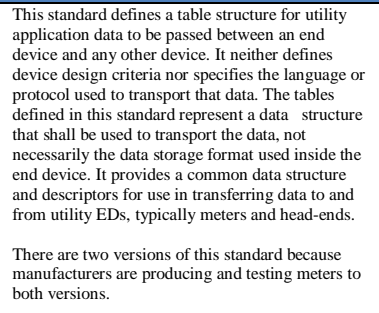 & 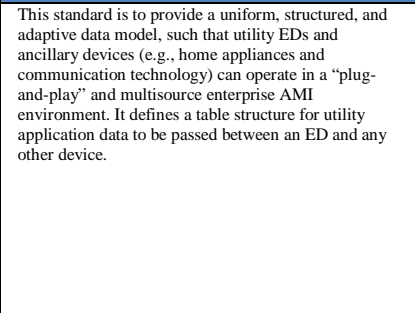 & & \multirow[t]{4}{*}{ 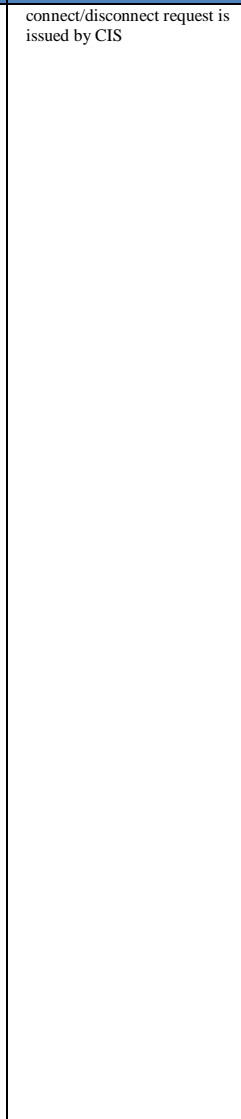 } & \\
\hline & 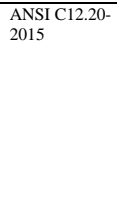 & 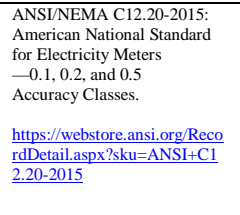 & & & 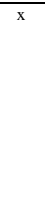 & & & & & 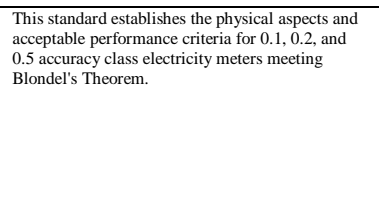 & 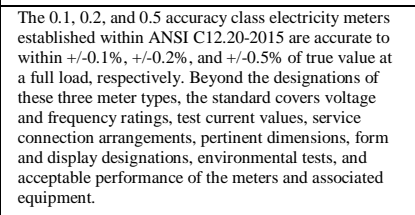 & & & \begin{tabular}{|l|}
$z$ \\
\end{tabular} \\
\hline & $\begin{array}{l}\text { ANSI C12.21- } \\
20066\end{array}$ & 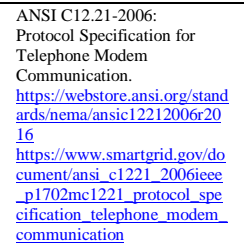 & $x$ & $\mathrm{x}$ & & & & & & 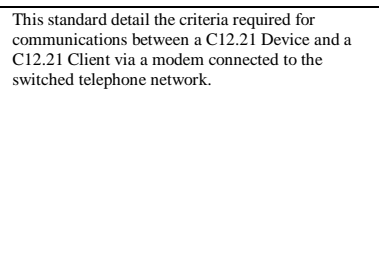 & 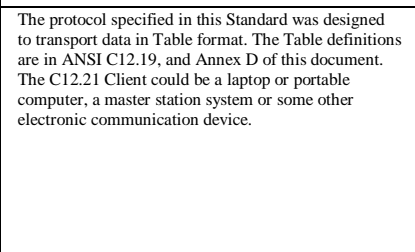 & & & \\
\hline & 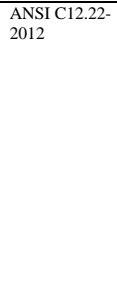 & 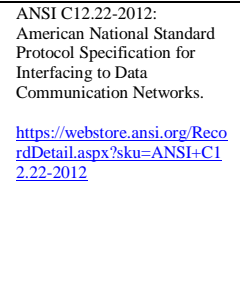 & $x$ & 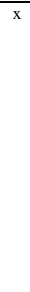 & & & & & \begin{tabular}{|l}
$x$ \\
\end{tabular} & 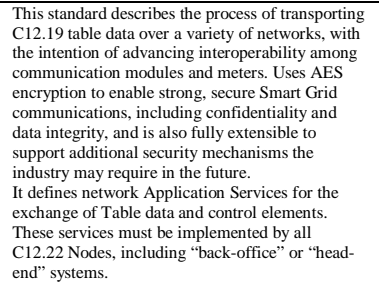 & 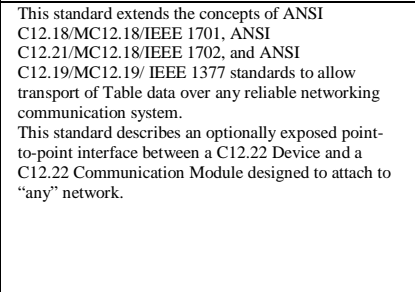 & & & \\
\hline
\end{tabular}




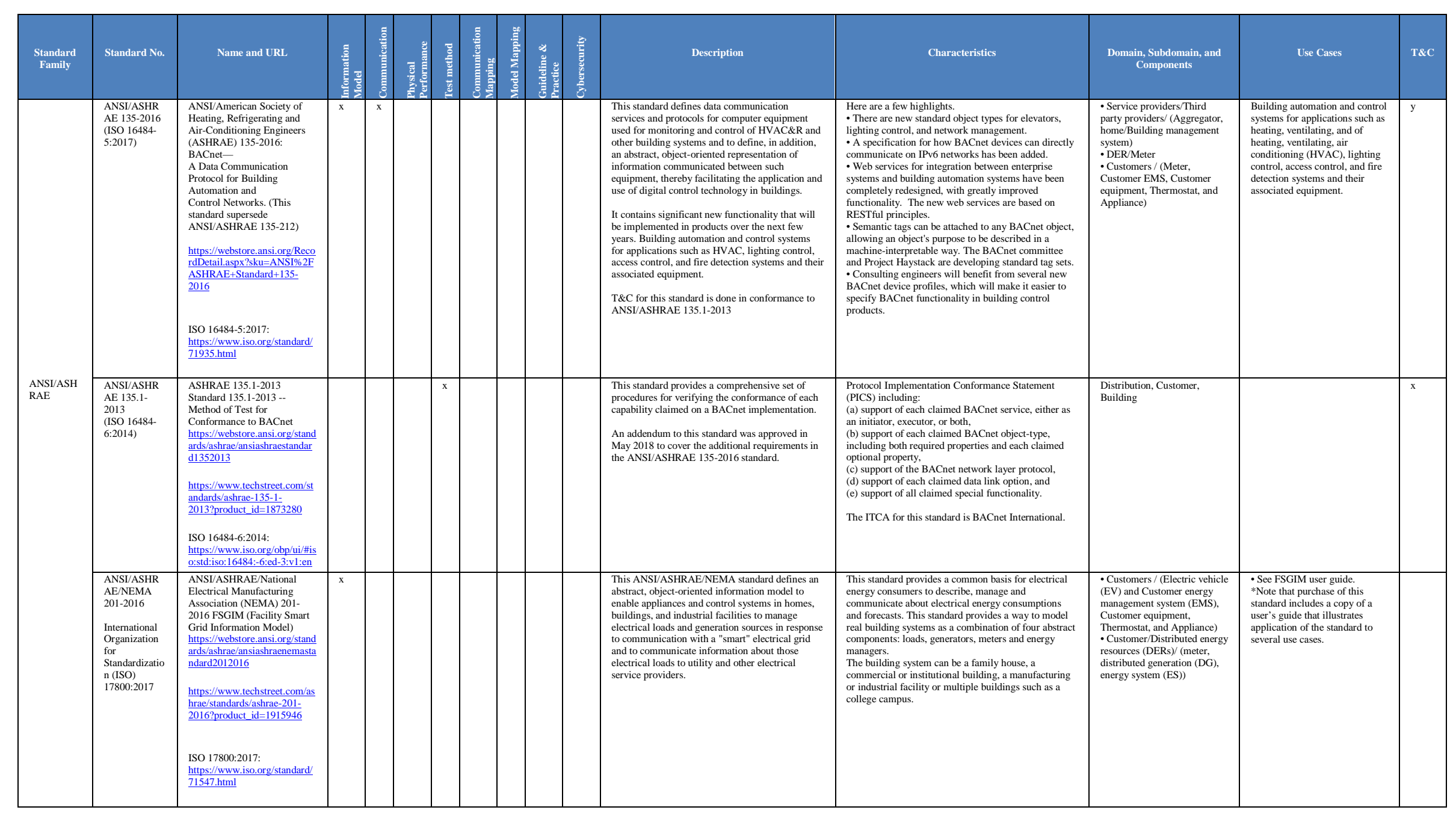




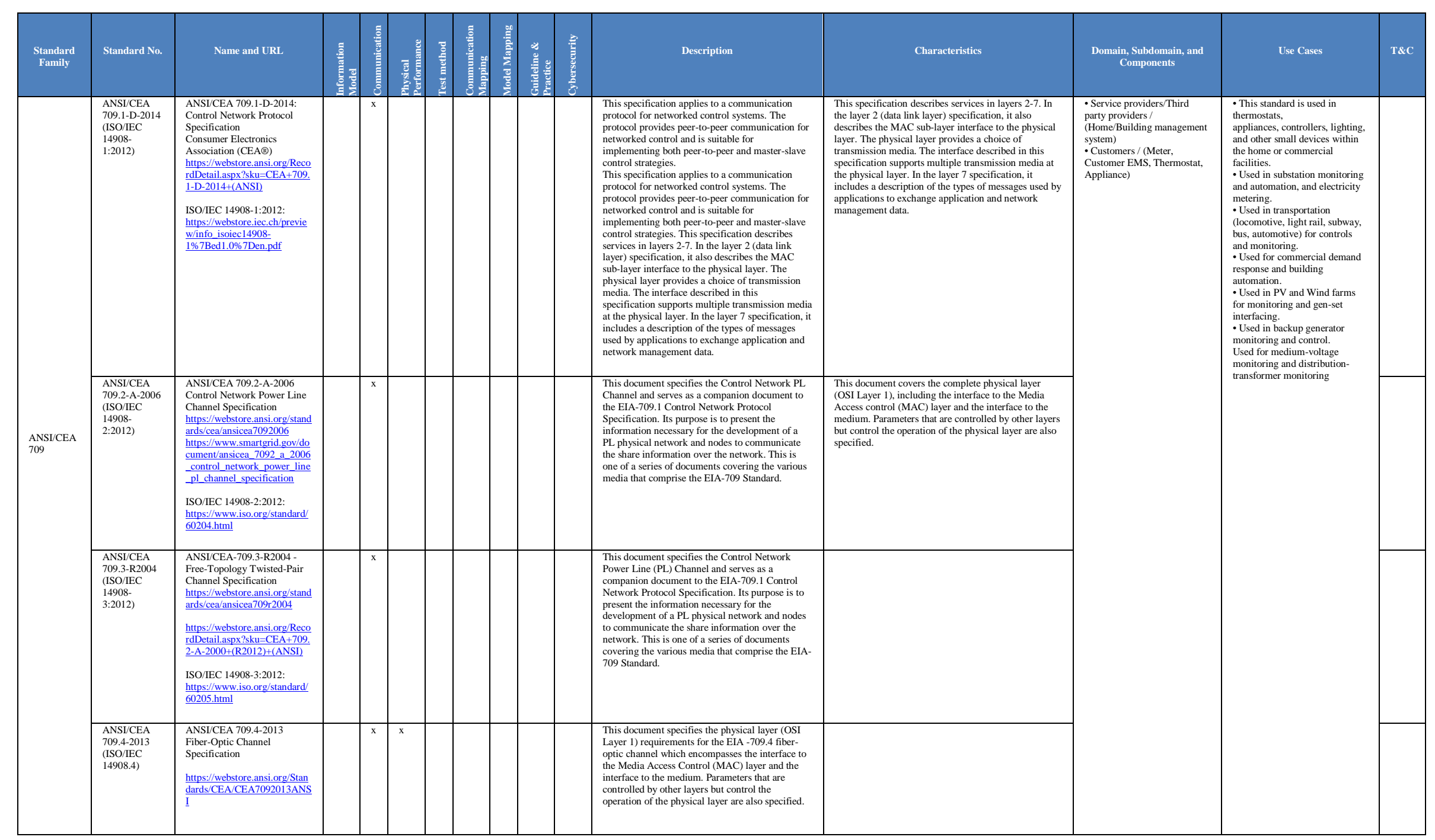




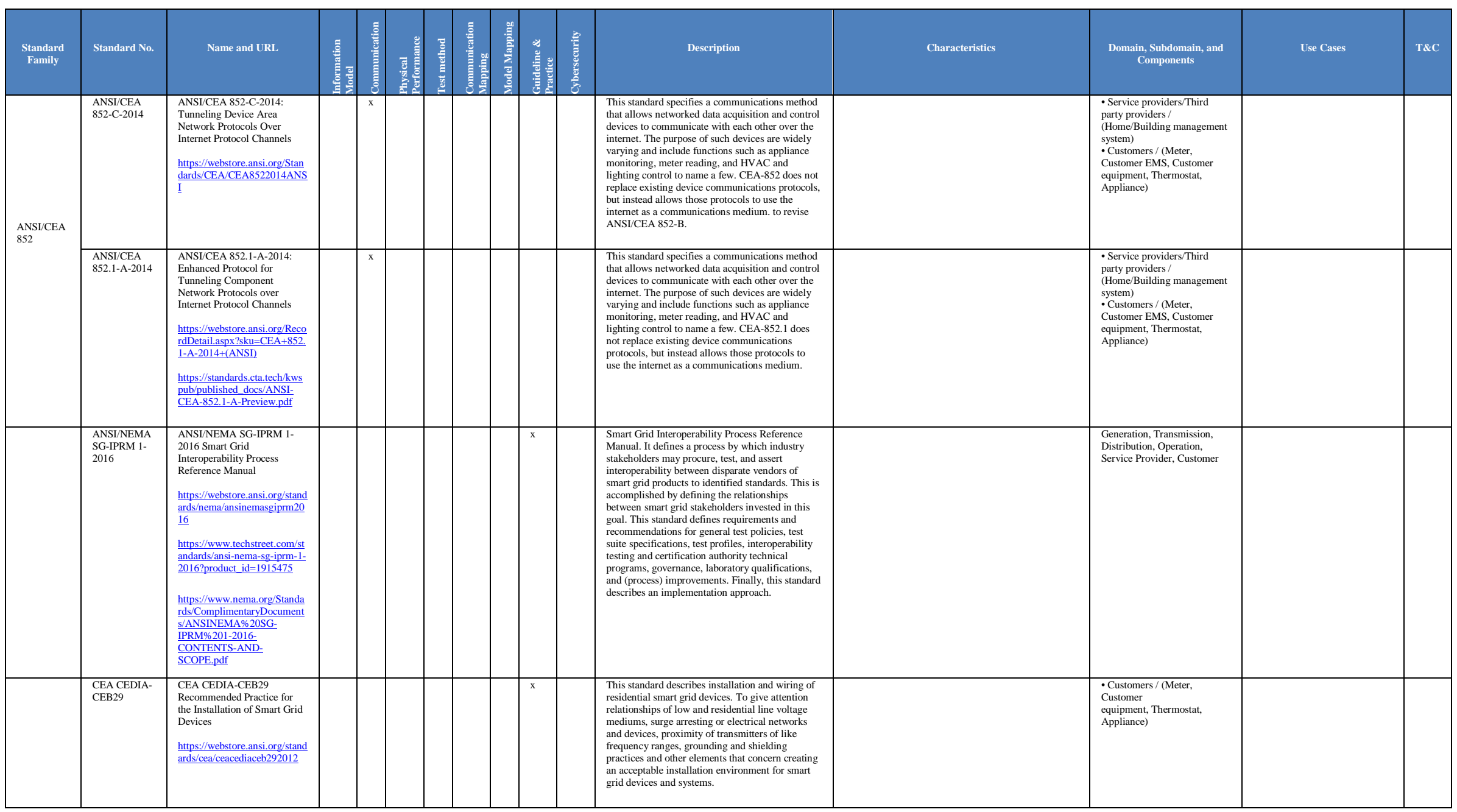




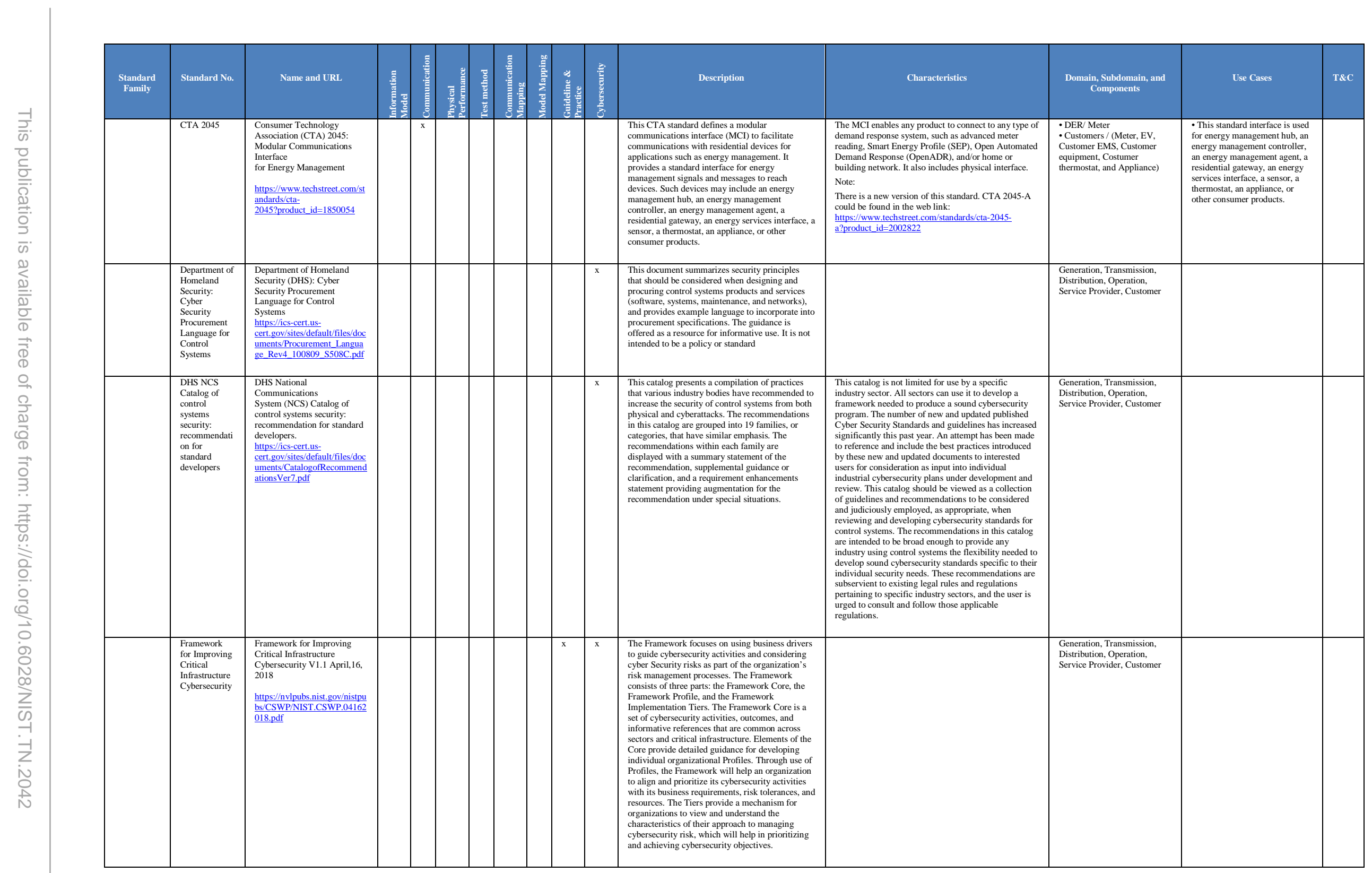




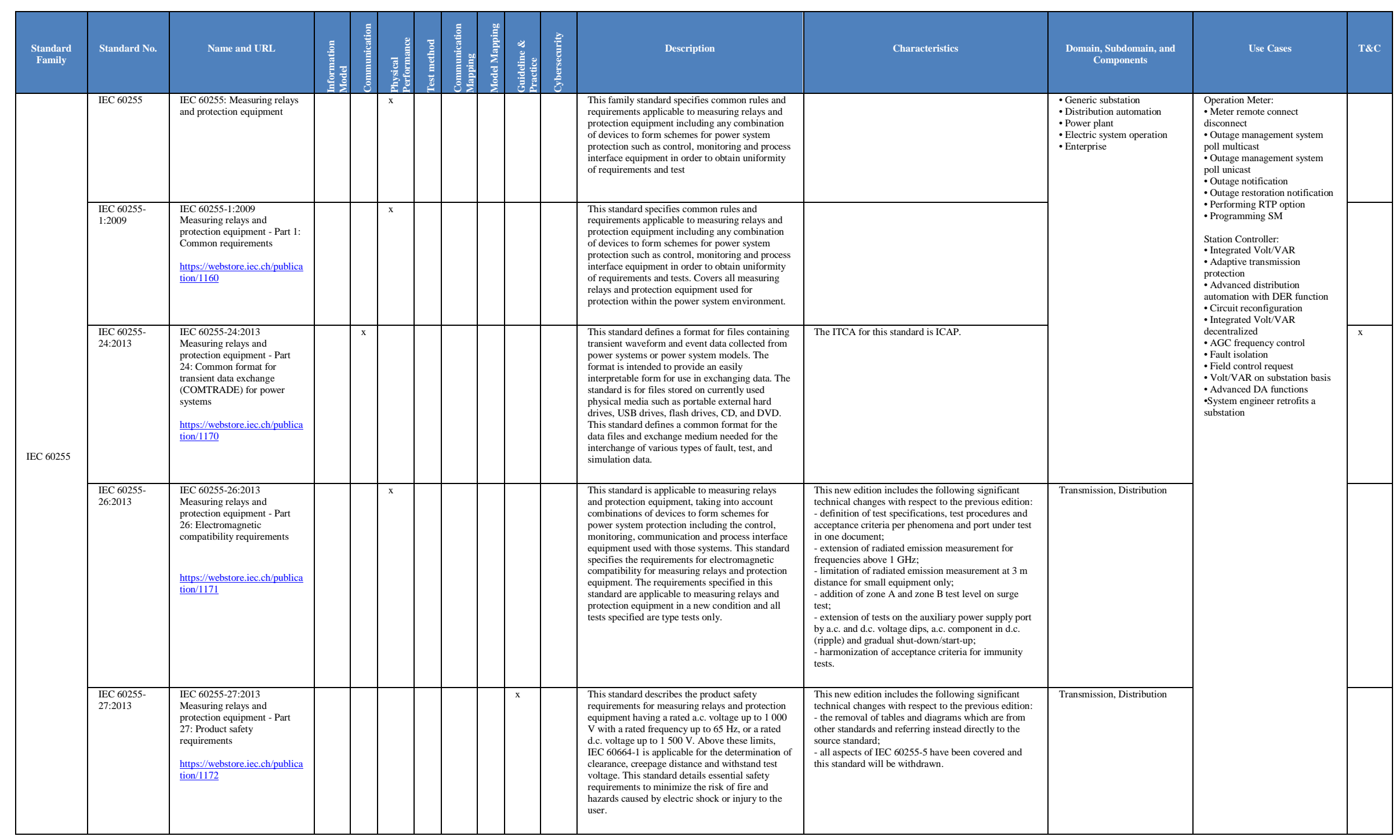




\begin{tabular}{|c|c|c|c|c|c|c|c|c|c|c|c|c|c|c|}
\hline $\begin{array}{c}\text { Standard } \\
\text { Family }\end{array}$ & Standard No. & Name and URL & 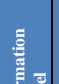 & & & 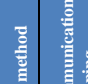 & 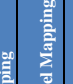 & 齐 & 差 & Description & Characteristics & $\begin{array}{l}\text { Domain, Subdomain, and } \\
\text { Components }\end{array}$ & Use Cases & т\&्C \\
\hline \multirow{4}{*}{$\begin{array}{c}\text { IEC } \\
60870-5\end{array}$} & $\begin{array}{l}\text { IEC 60870-5- } \\
\text { 101:2003- }\end{array}$ & 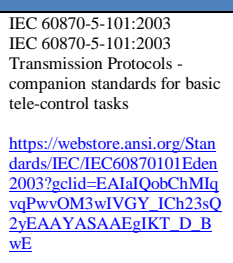 & & $\mathrm{x}$ & & & & & & 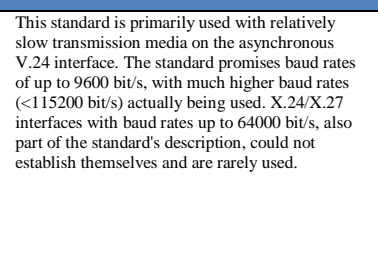 & The ITCA for this standard is DNV GL. & \begin{tabular}{|l}
-Among EMS, supervisory \\
control and data acuuistion \\
(SCADA), EMS, wide area \\
managenent systems \\
(WAMS)
\end{tabular} & & \begin{tabular}{|l|l|l|l|}
$x$ \\
\end{tabular} \\
\hline & $\begin{array}{l}\text { IEC 60870-5- } \\
\text { 102:1996-5 }\end{array}$ & 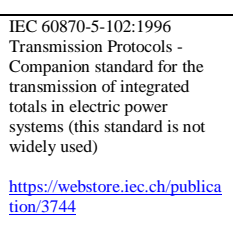 & & $\mathrm{x}$ & & & & & & 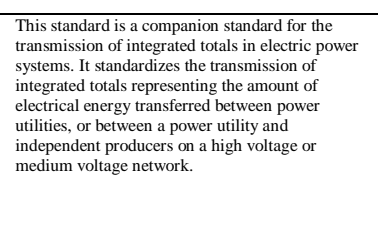 & 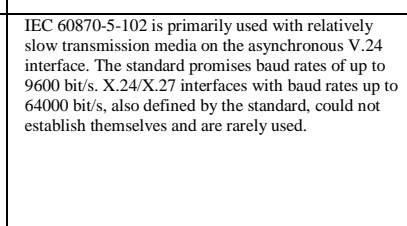 & $\begin{array}{l}\text { Among EMS, SCADA, } \\
\text { EMS, WAMS systems }\end{array}$ & \begin{tabular}{|l}
-The transmisision of integrated \\
totals in EPS
\end{tabular} & \\
\hline & $\begin{array}{l}\text { IEC 60870-5- } \\
\text { 103:1997 }\end{array}$ & 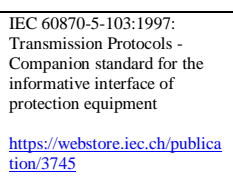 & & $\mathrm{x}$ & & & & & & 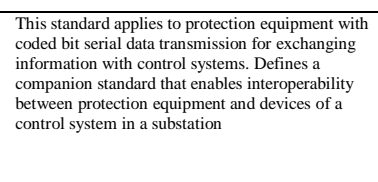 & & $\begin{array}{l}\text {-Among EMS, SCADA, } \\
\text { EMS, WAMS systems }\end{array}$ & 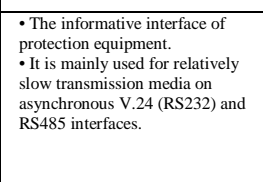 & \\
\hline & 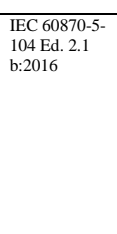 & 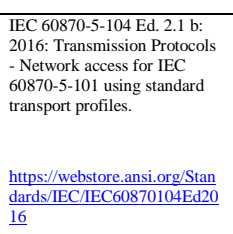 & & $\mathrm{x}$ & & & & & & 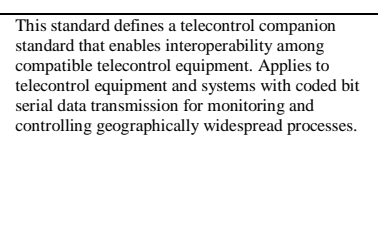 & 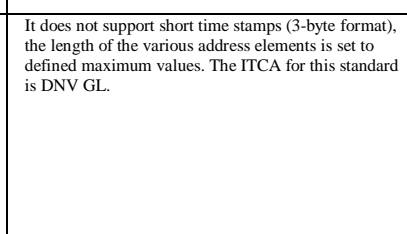 & $\begin{array}{l}\text { Among EMS, SCADA, } \\
\text { EMS, WAMS systems }\end{array}$ & 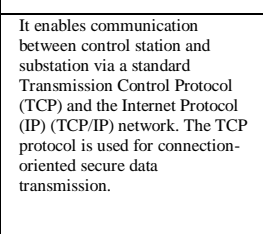 & \begin{tabular}{|l|l|l}
$x$ \\
\end{tabular} \\
\hline
\end{tabular}




\begin{tabular}{|c|c|c|c|c|c|c|c|c|c|c|c|c|c|c|}
\hline $\begin{array}{l}\text { Standard } \\
\text { Family }\end{array}$ & Standard No. & Name and URL & & & & 赔 & $\mid$ & $\mid$ & 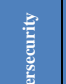 & Description & Characteristics & $\begin{array}{l}\text { Domain, Subdomain, and } \\
\text { Components }\end{array}$ & Use Cases & TRC \\
\hline \multirow{3}{*}{$\begin{array}{l}\text { IEC } \\
\text { IE870-6 }\end{array}$} & $\begin{array}{l}\text { IEC 60870-6- } \\
\text { 500:2014 }\end{array}$ & 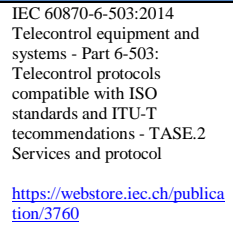 & & & & & & & & 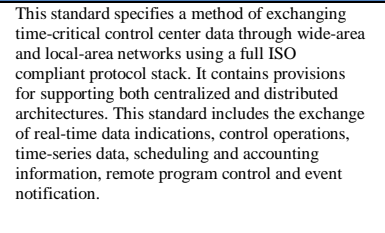 & 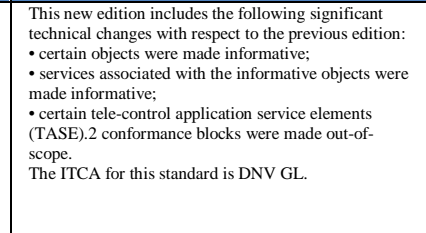 & 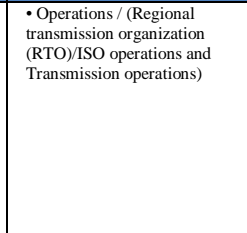 & 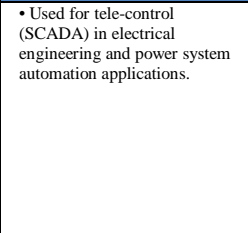 & $x$ \\
\hline & $\begin{array}{l}\text { IEC 60870-6- } \\
702: 2014\end{array}$ & 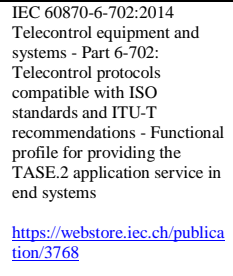 & & $\bar{x}$ & & & & & & 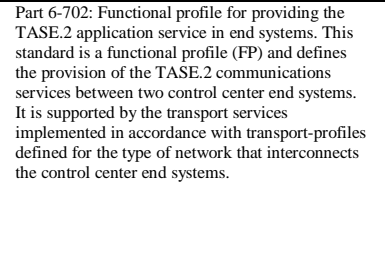 & 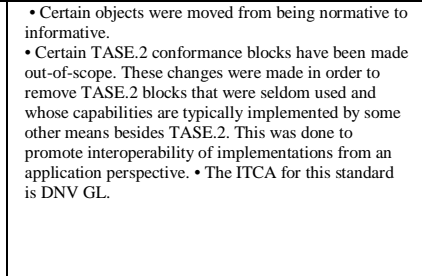 & $\begin{array}{l}\text { Opperations / (RTT/ISO } \\
\text { operations and Transmission } \\
\text { operations) }\end{array}$ & 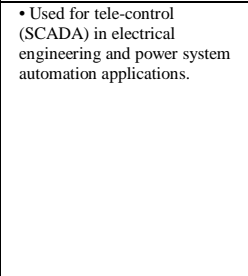 & $x$ \\
\hline & $\begin{array}{l}\text { IEC 60870-6- } \\
\text { 802:2014 }\end{array}$ & 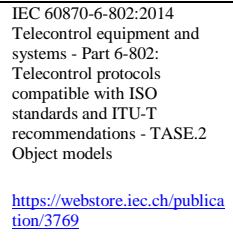 & $x$ & & & & & & & 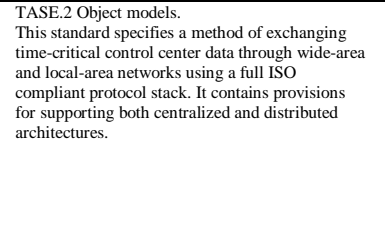 & 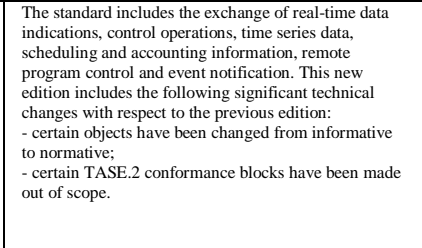 & 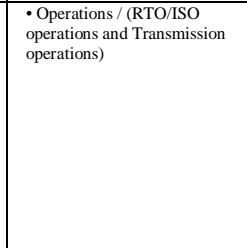 & 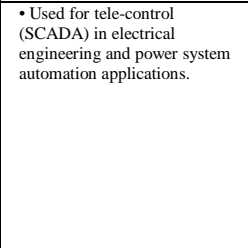 & $x$ \\
\hline
\end{tabular}




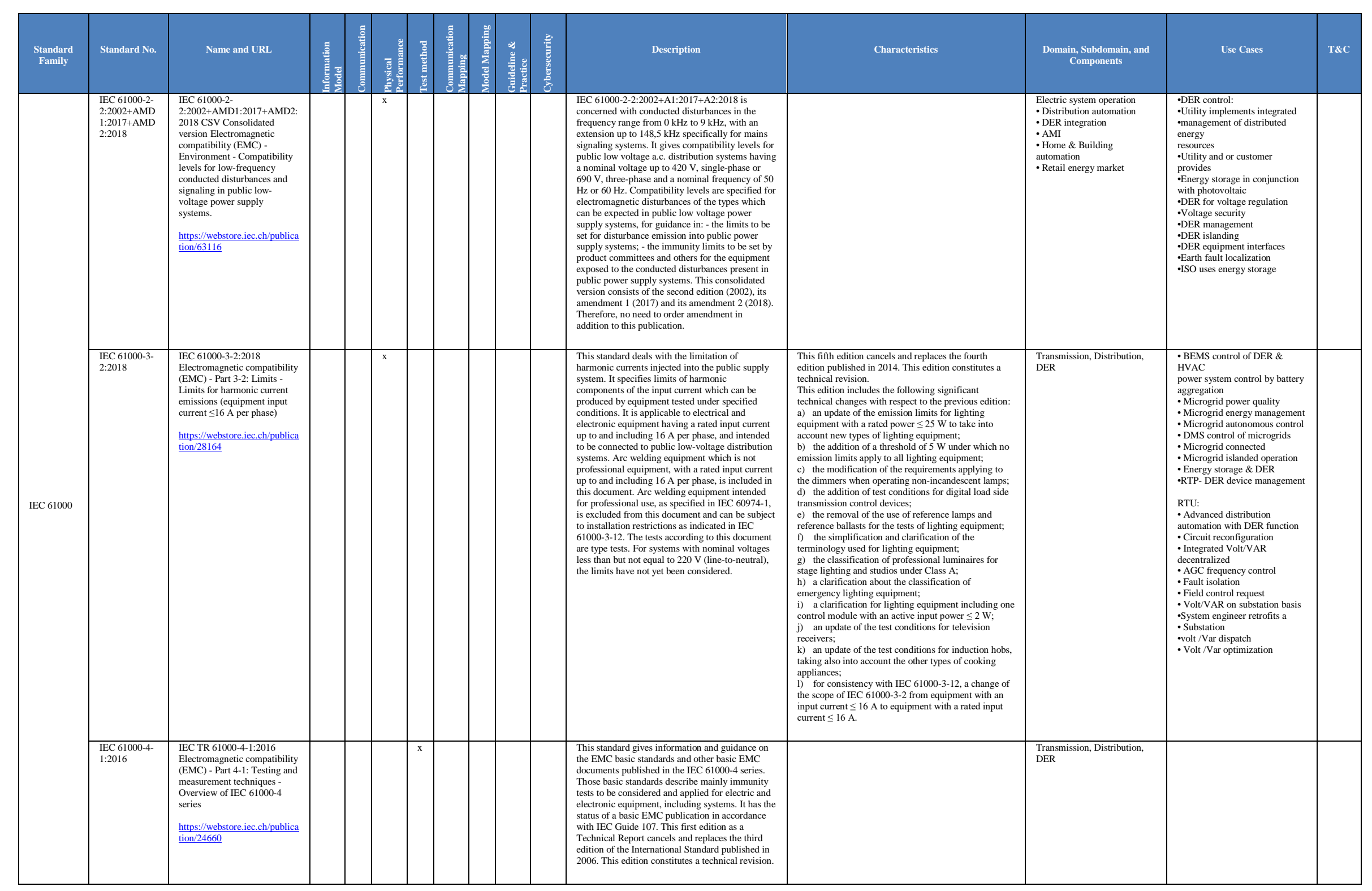




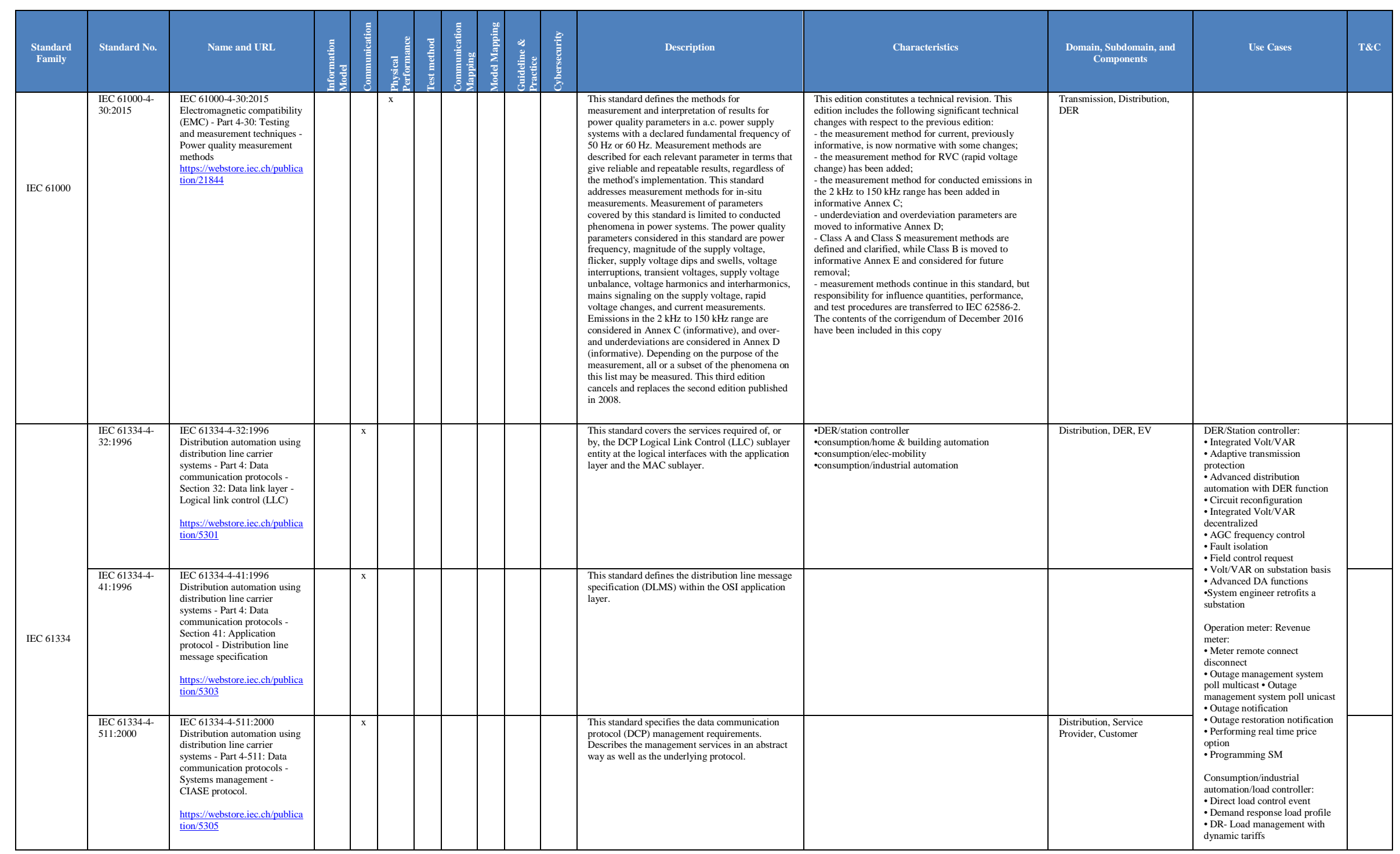




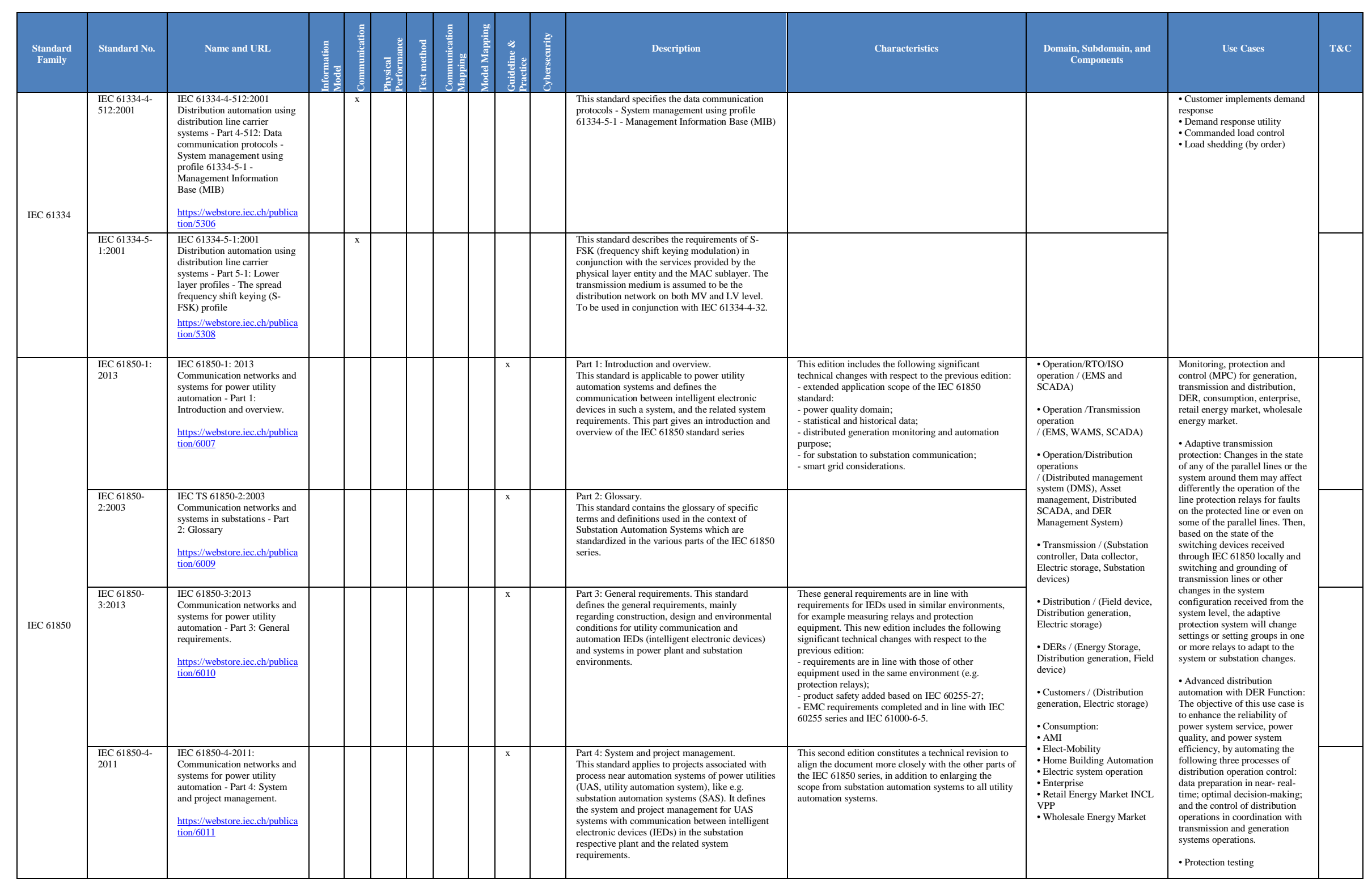




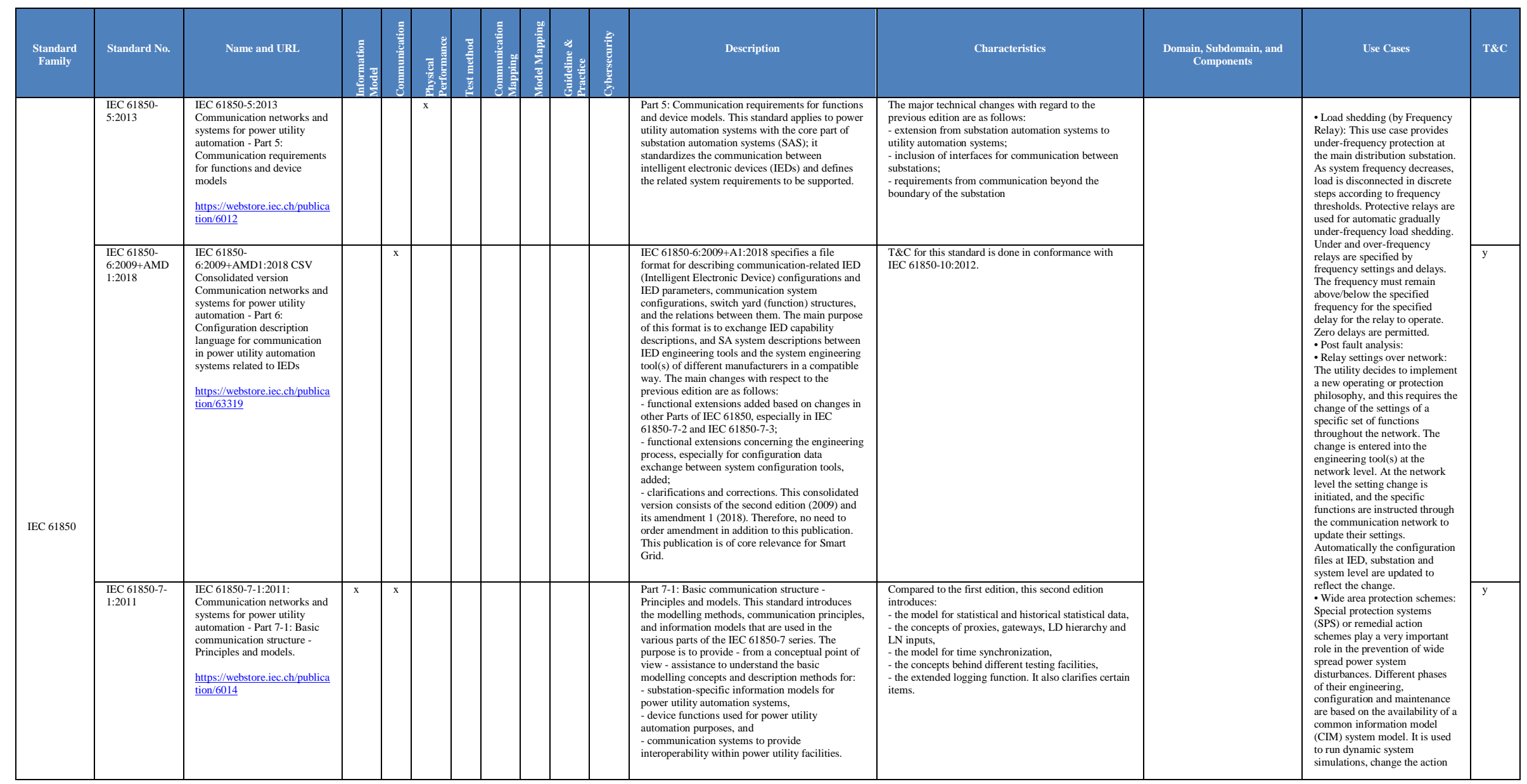




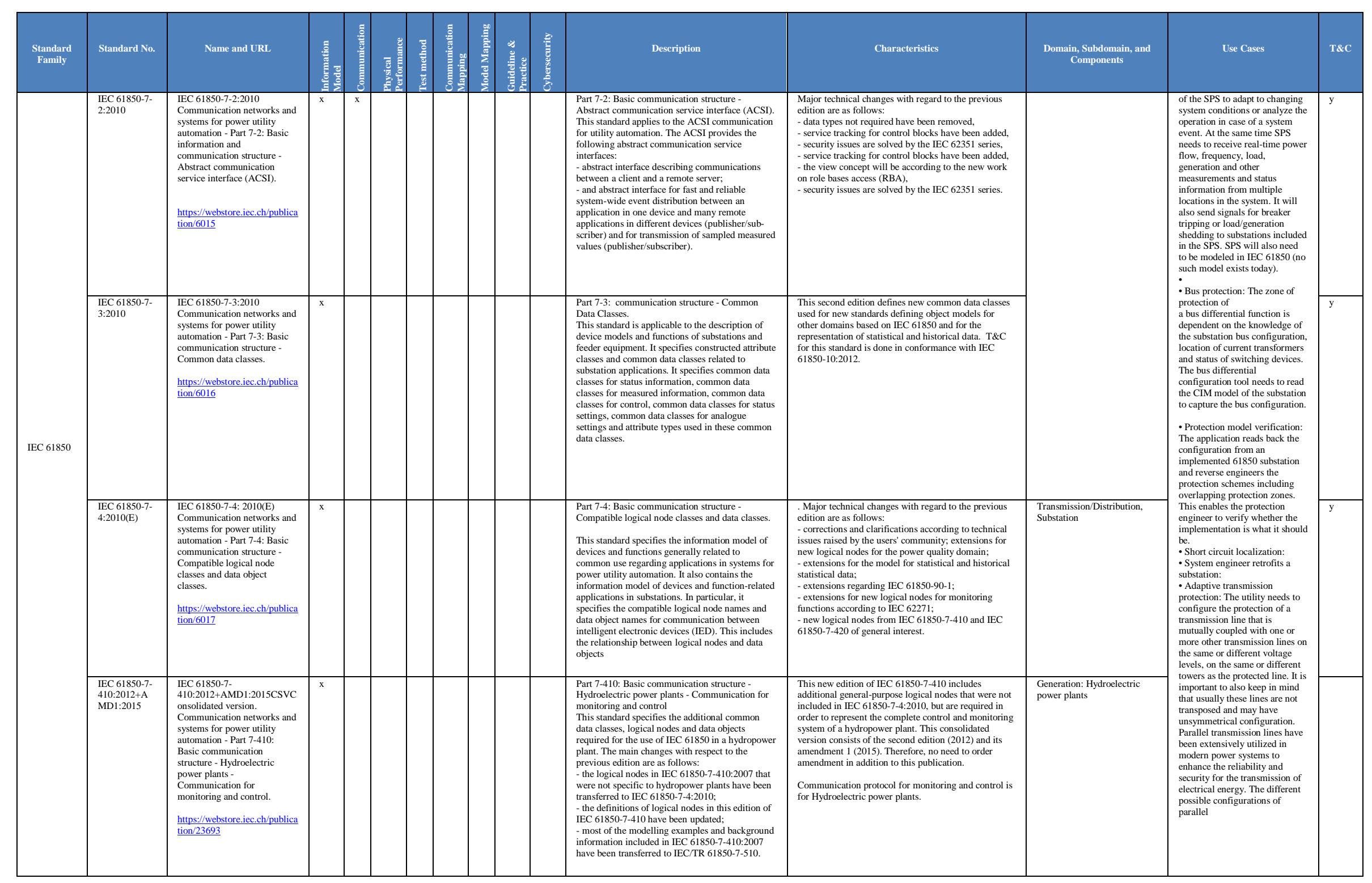




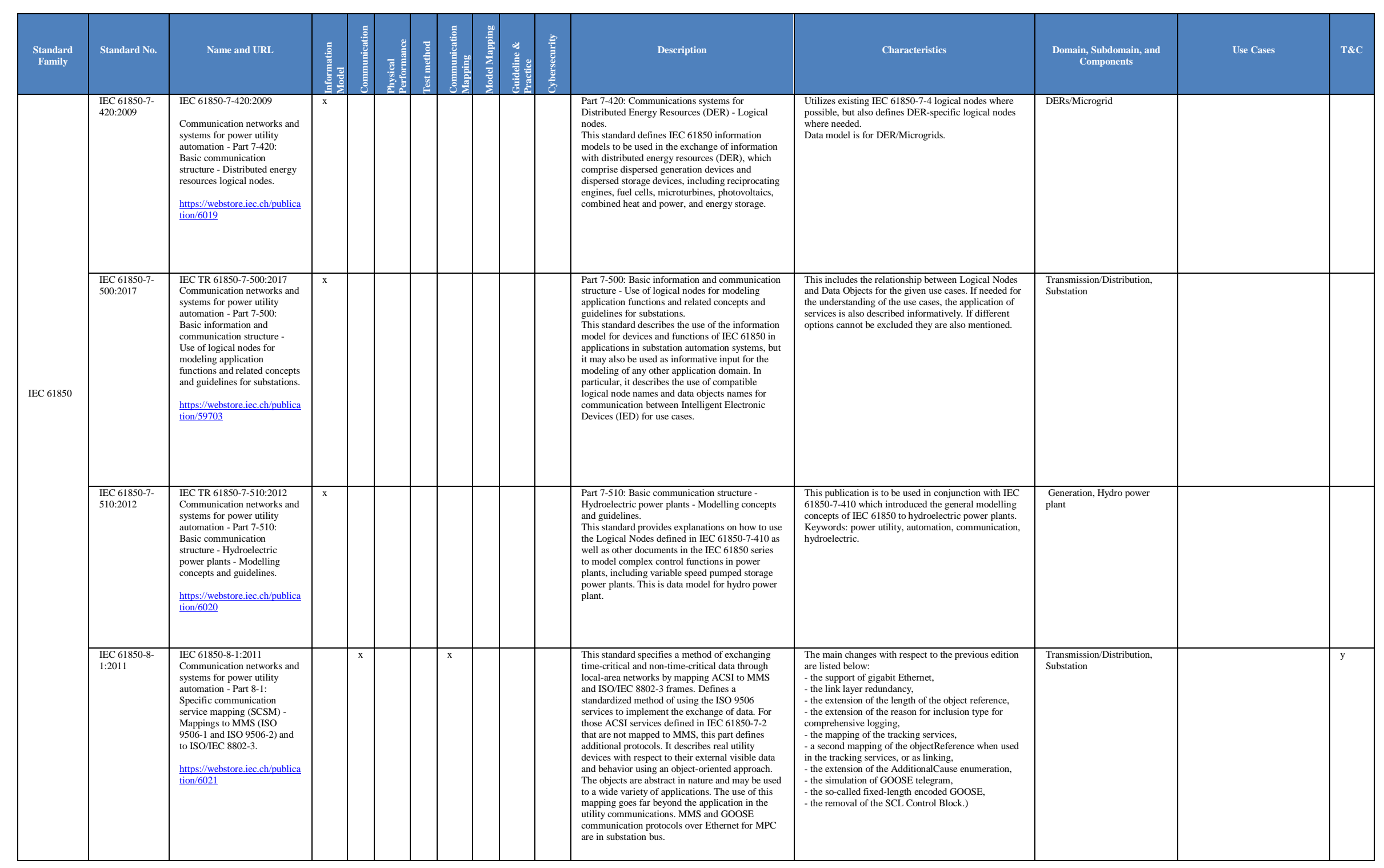




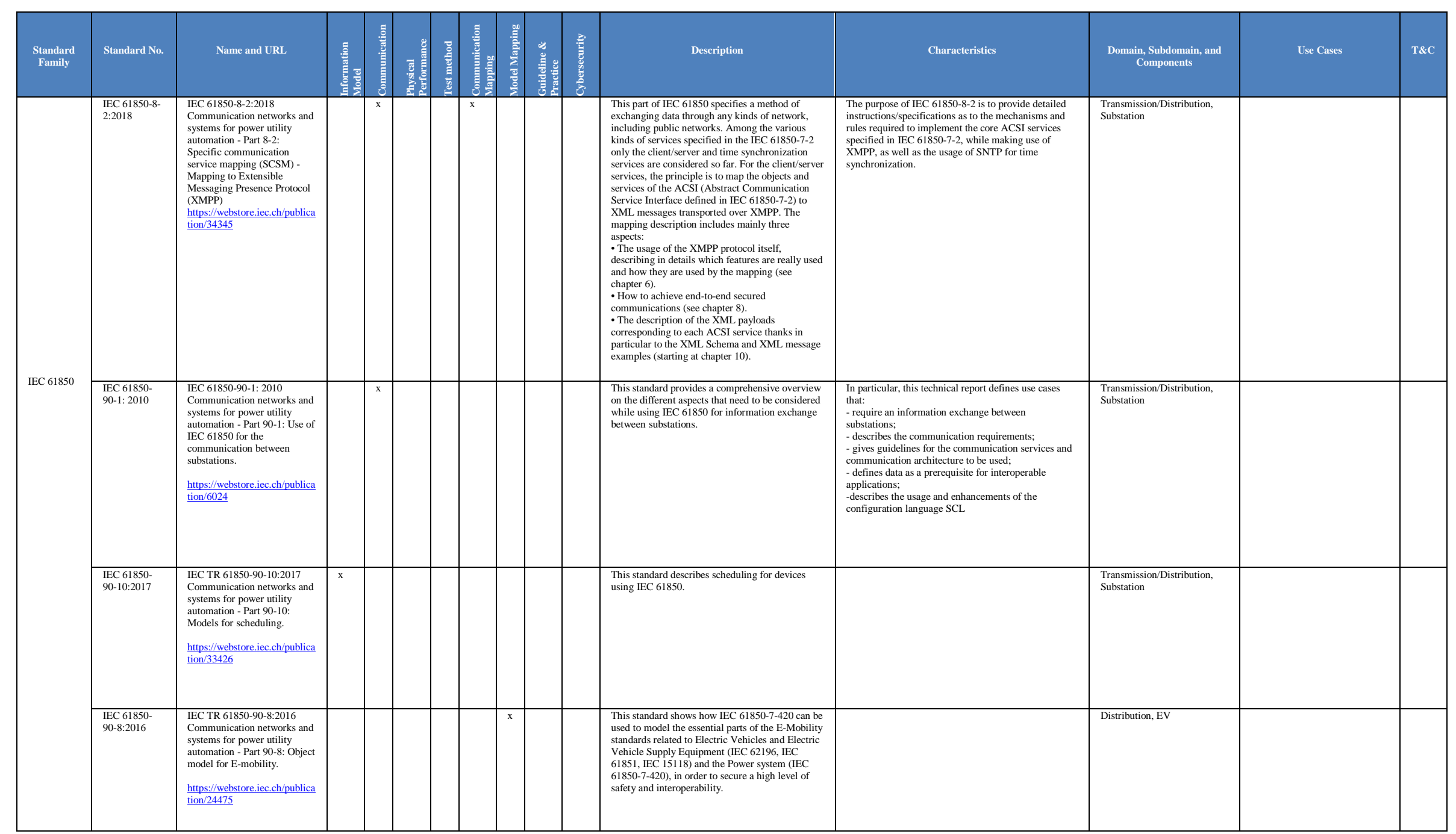




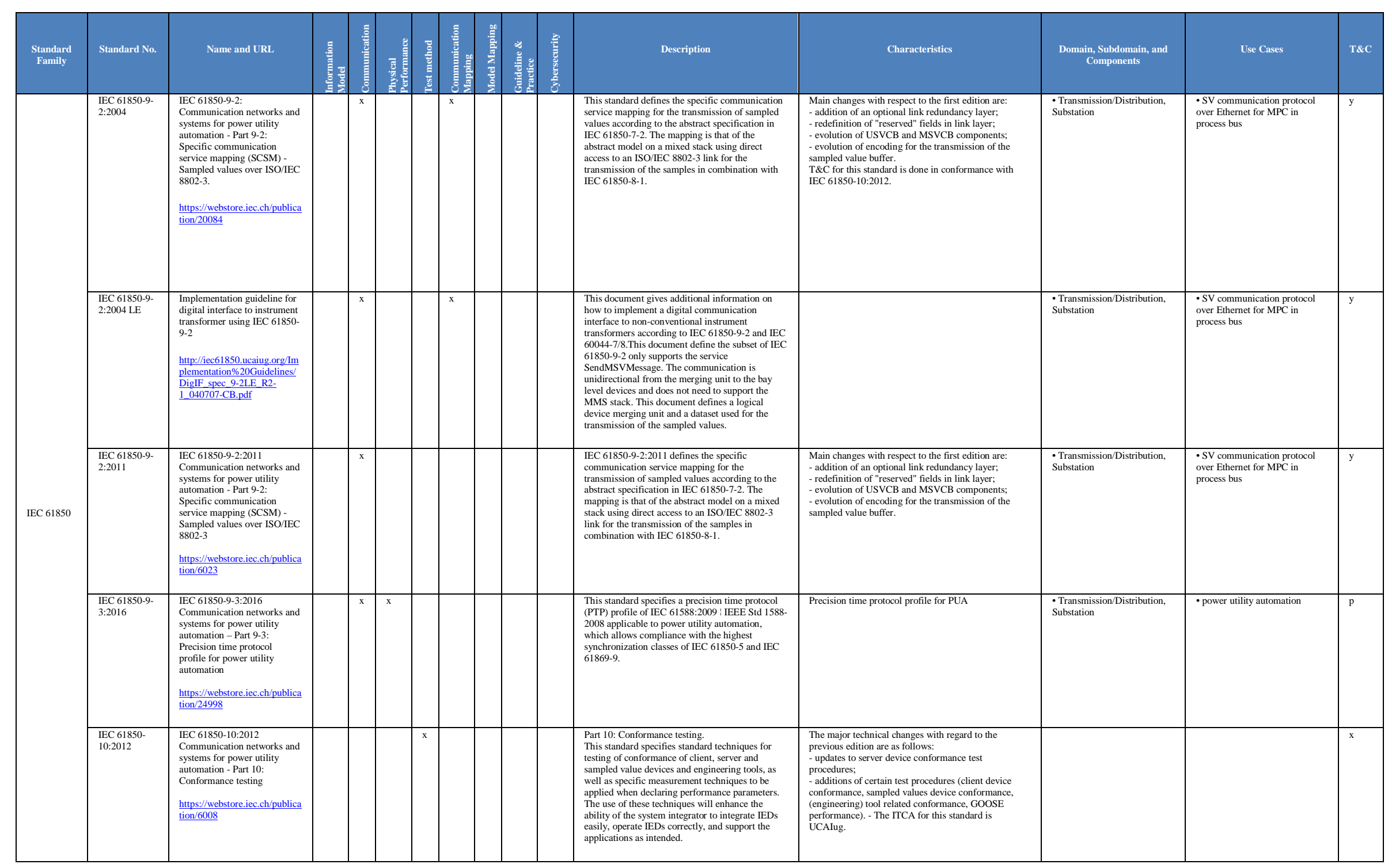




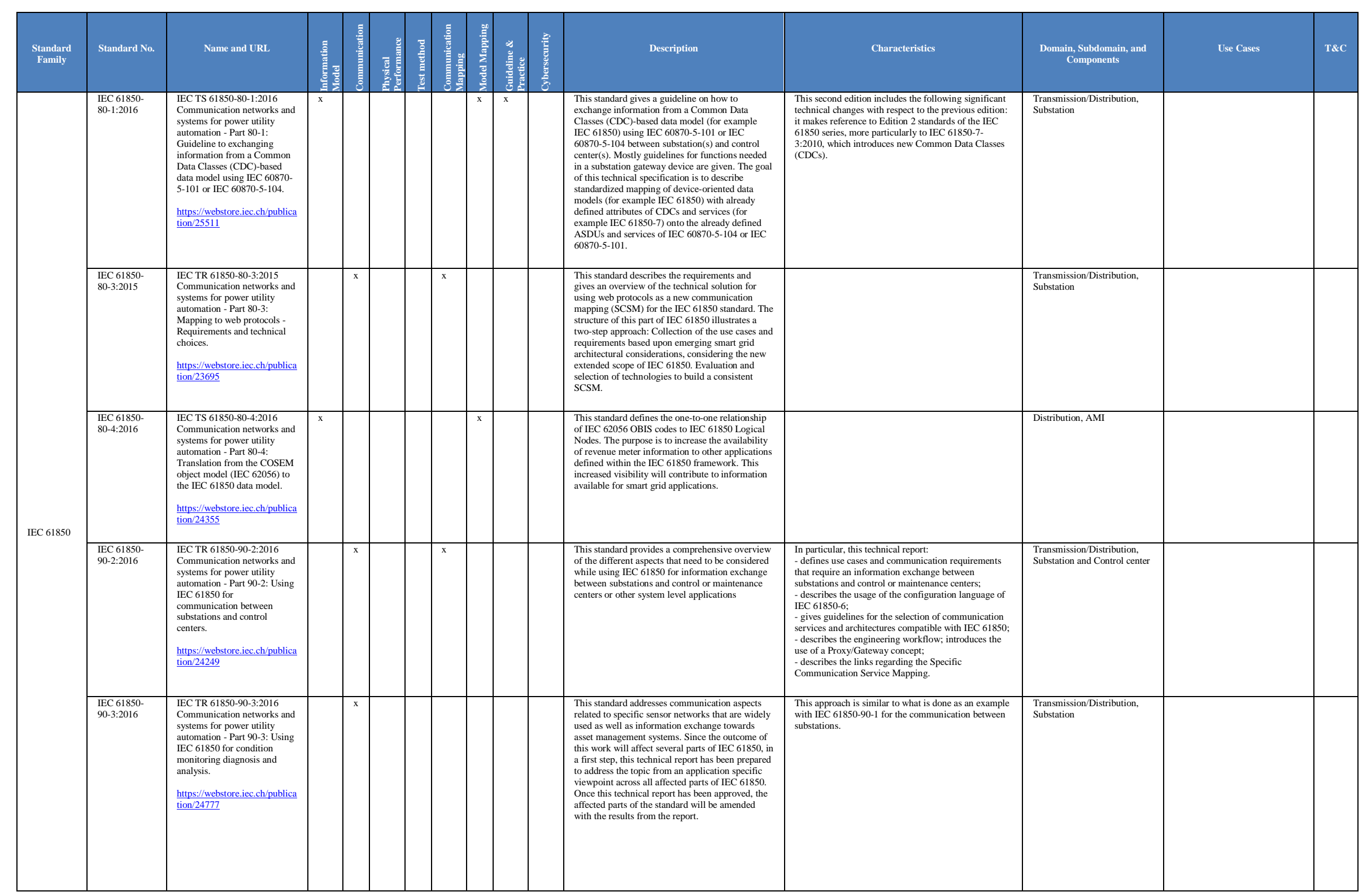




\begin{tabular}{|c|c|c|c|c|c|c|c|c|c|c|c|c|c|c|}
\hline $\begin{array}{c}\text { Standard } \\
\text { Family }\end{array}$ & Standard No. & Name and URL & & & - & & & & & Description & Characterisitics & $\begin{array}{l}\text { Domain, Subdomaini, and } \\
\text { Components }\end{array}$ & Use Cases & T\&C \\
\hline \multirow{4}{*}{ IEC 61850} & $\begin{array}{l}\text { IEC 61850- } \\
90-4: 2013\end{array}$ & 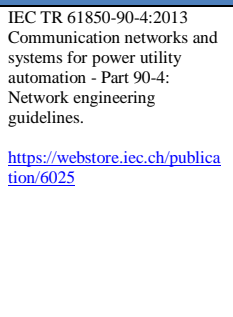 & & & & & & & $\bar{x}$ & 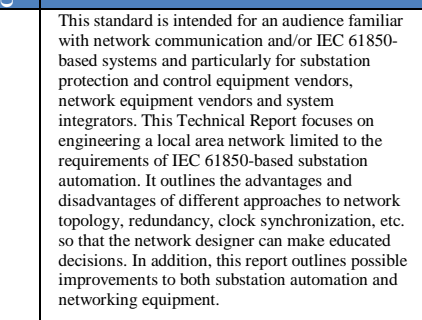 & 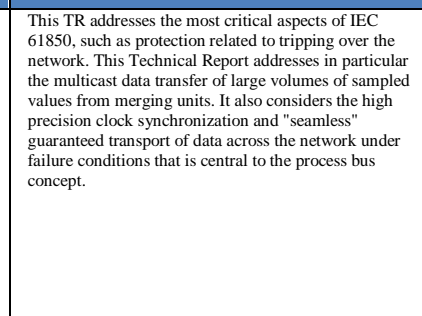 & 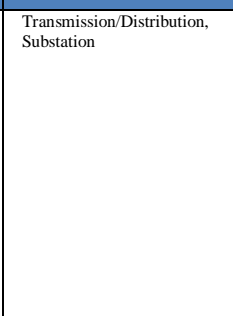 & & \\
\hline & $\begin{array}{l}\text { IEC 61850- } \\
\text { 90-5:2012 }\end{array}$ & 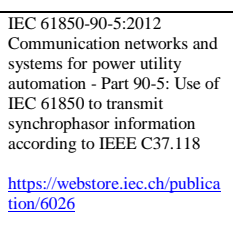 & $x$ & & & 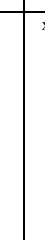 & & & & 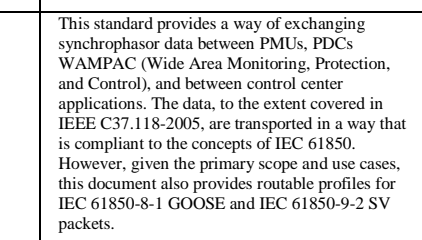 & 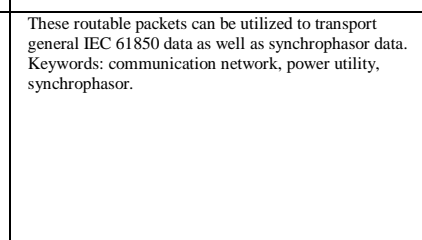 & \begin{tabular}{|l|l|} 
Transmissiononistribution, \\
Substation
\end{tabular} & 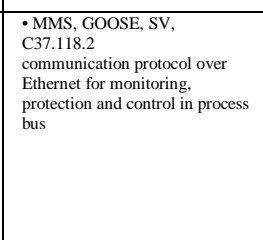 & \\
\hline & $\begin{array}{l}\text { IEC 61850- } \\
{ }_{90-7: 2013}\end{array}$ & 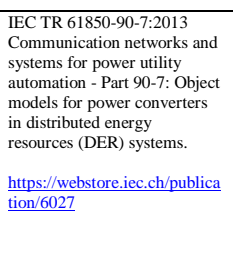 & $\mathrm{x}$ & & & & & & & 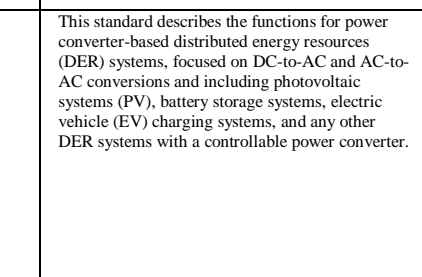 & 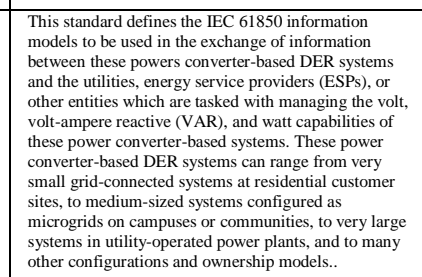 & \begin{tabular}{|l|} 
Transmission, Distribution, \\
DER
\end{tabular} & 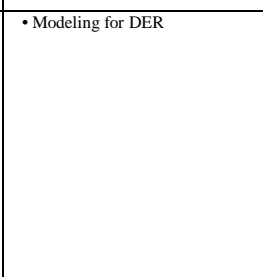 & \\
\hline & $\begin{array}{l}\text { IEC 61850- } \\
\text { 90-12:2015 }\end{array}$ & 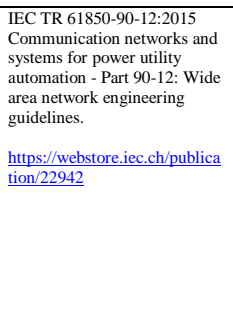 & & & & & & & & 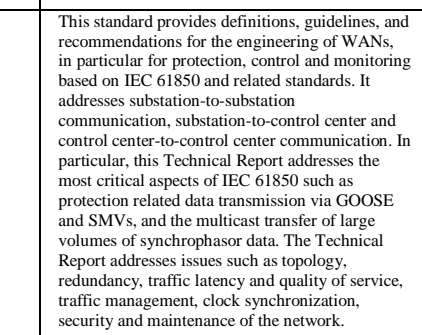 & 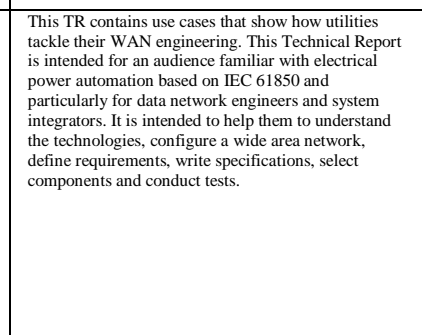 & \begin{tabular}{|l} 
Transmisisiond Distribution, \\
Suustation
\end{tabular} & & \\
\hline
\end{tabular}




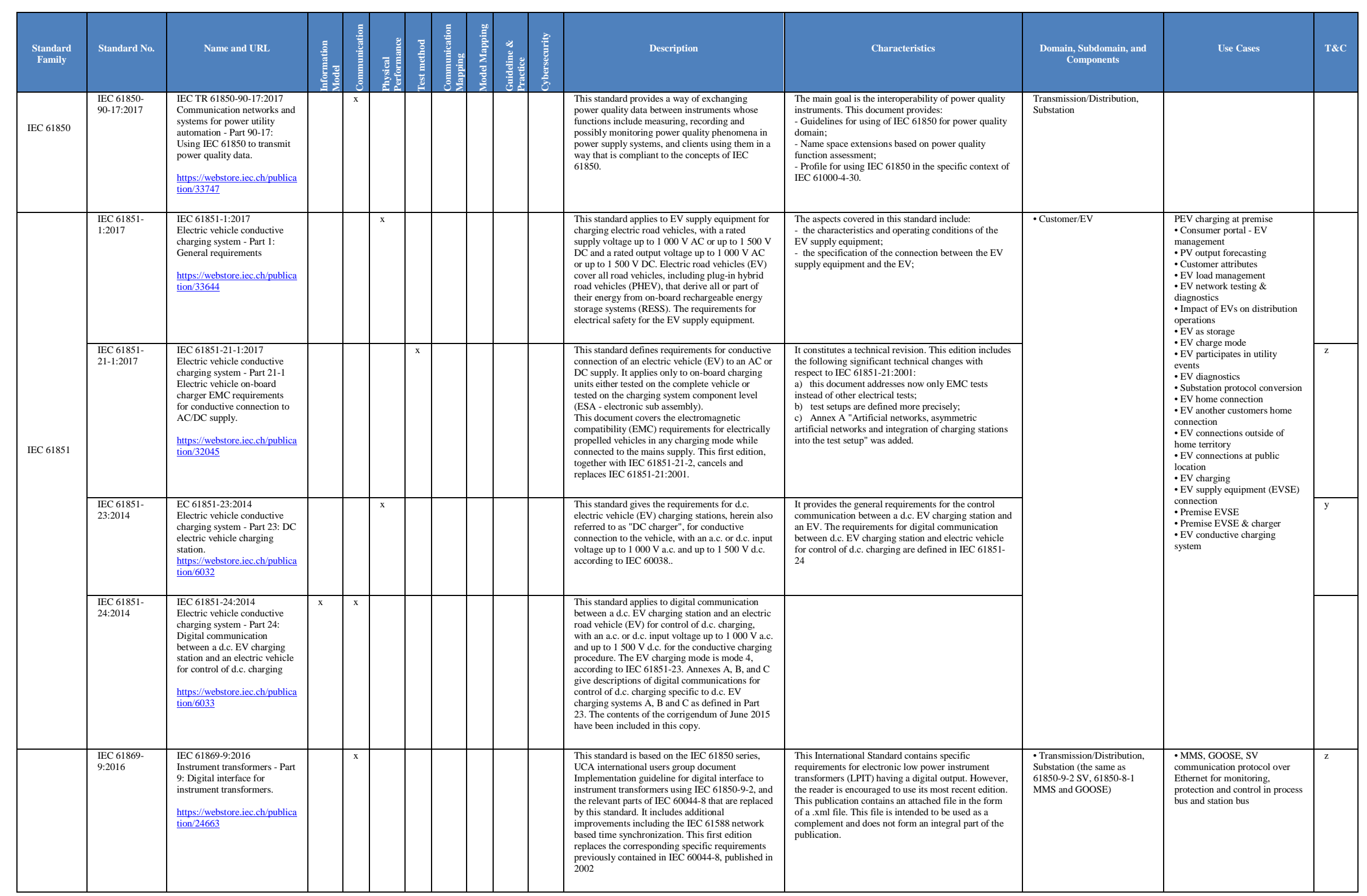




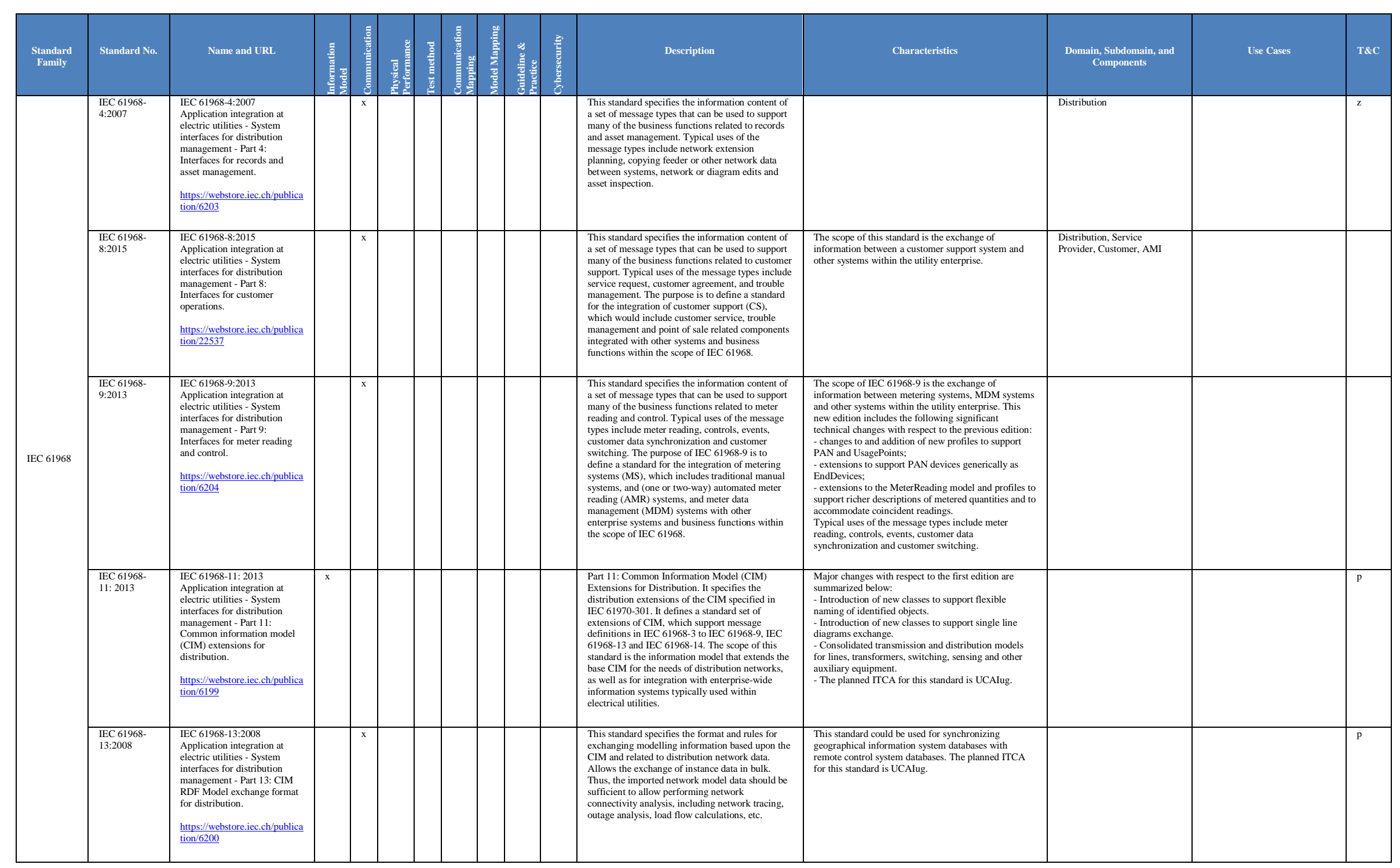




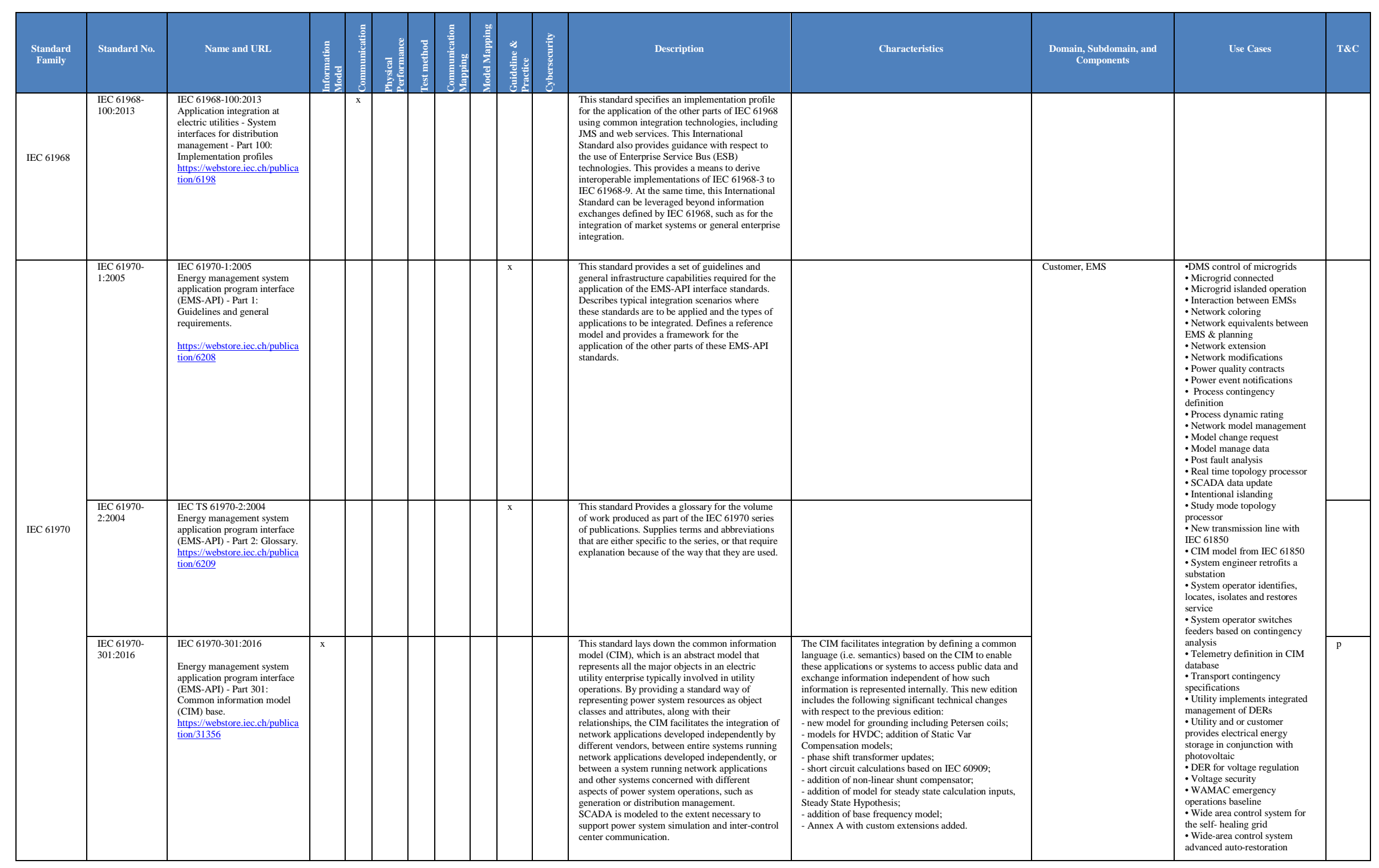




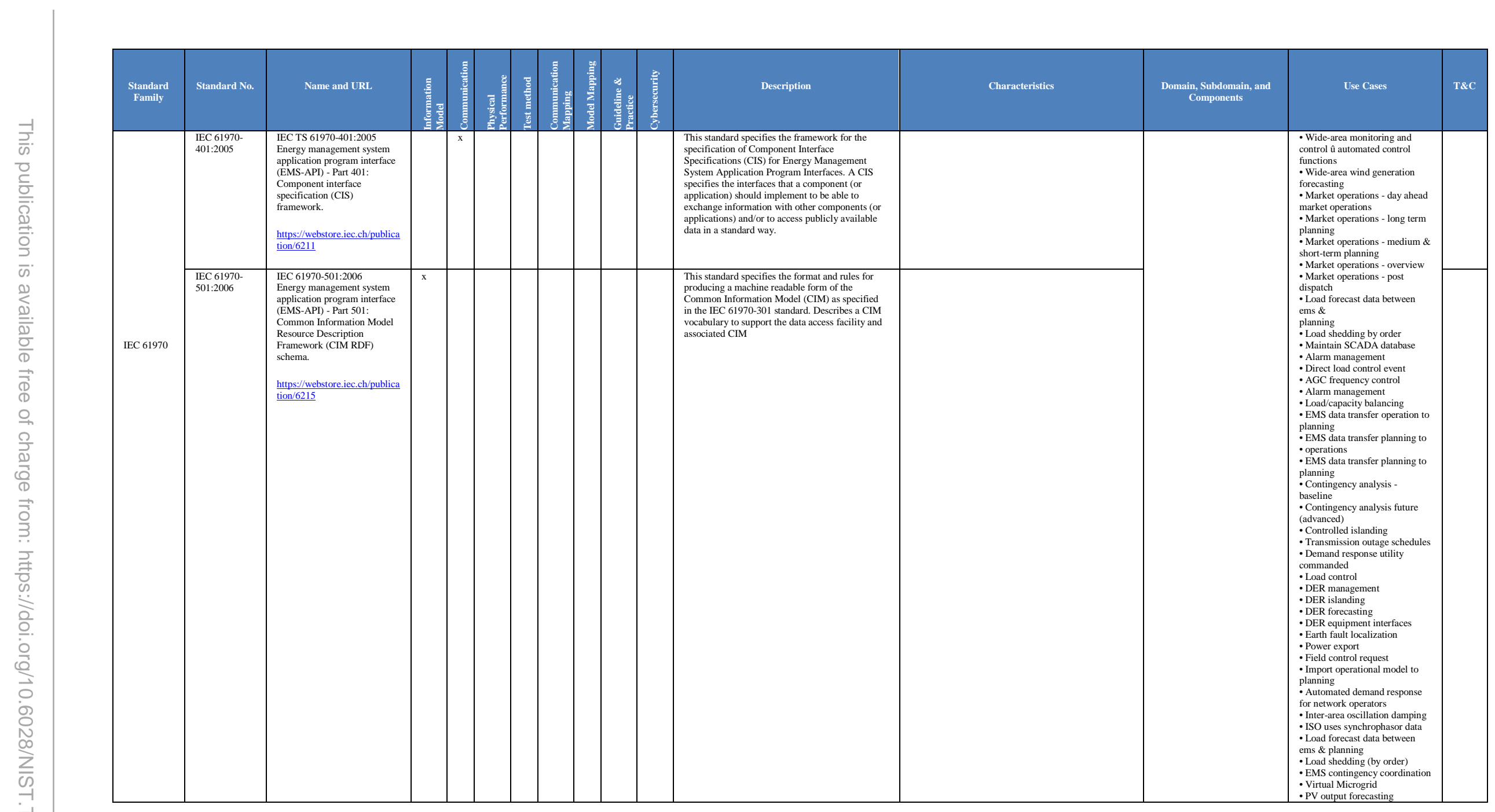




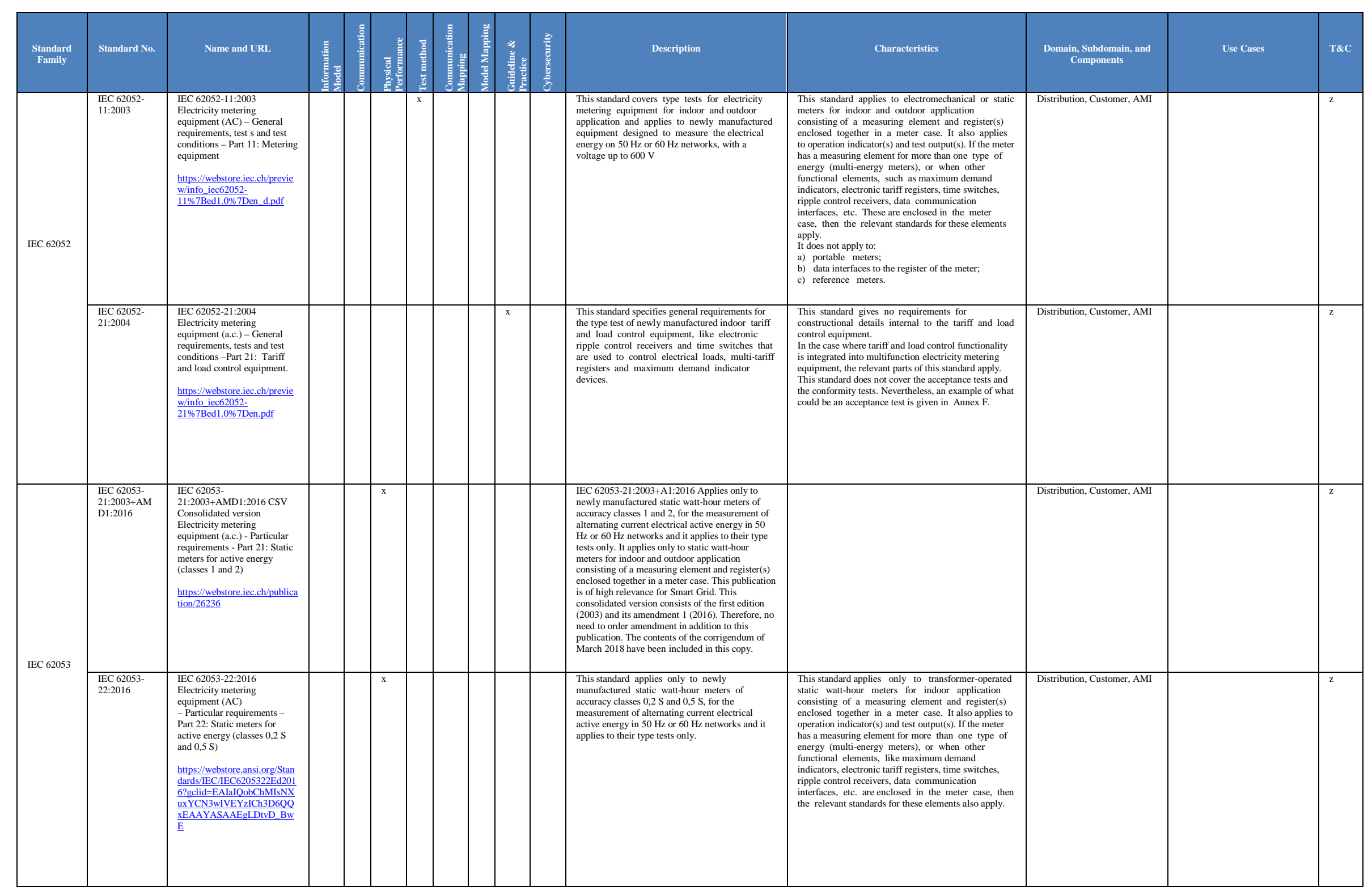




\begin{tabular}{|c|c|c|c|c|c|c|c|c|c|c|c|c|c|c|}
\hline $\begin{array}{c}\text { Standardd } \\
\text { Fanily }\end{array}$ & Standard No. & Name and URL & $\bar{z}$ & & & 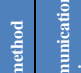 & & 尊 & 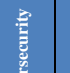 & Description & Characteristics & $\begin{array}{l}\text { Domain, Subdomanin, and } \\
\text { Components }\end{array}$ & Use Cases & $\mathrm{T} \& \mathrm{C}$ \\
\hline IEC 62053 & IEC 62053-23 & 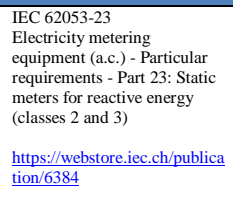 & & & k & & & & & 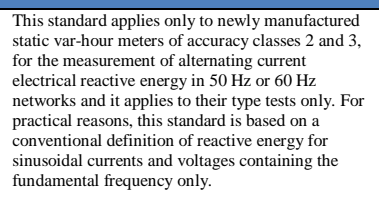 & & Distribution, Customer, AMI & & $\mathrm{z}$ \\
\hline IEC 62054 & IEC 62054-21 & 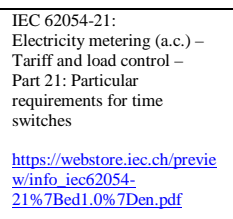 & & & & & & & & 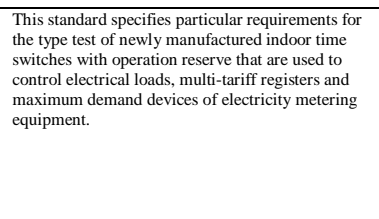 & & Distribution, Customer, AMI & & $z$ \\
\hline \multirow[t]{2}{*}{ IEC 62056} & $\begin{array}{l}\text { IEC 62056-1- } \\
0: 2014\end{array}$ & 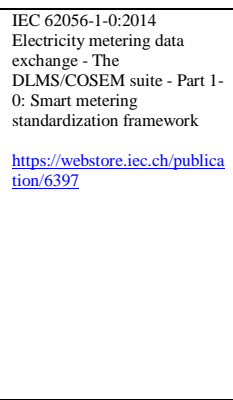 & & & & & & $\mathrm{x}$ & & 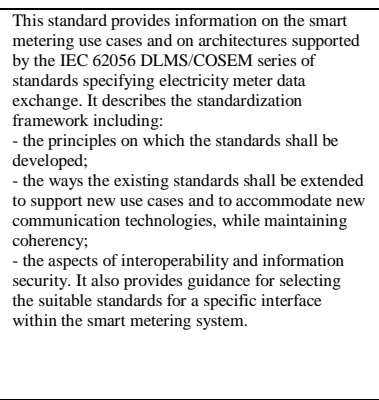 & & 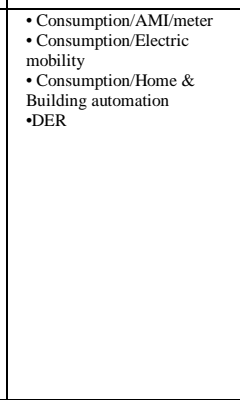 & \multirow{2}{*}{ 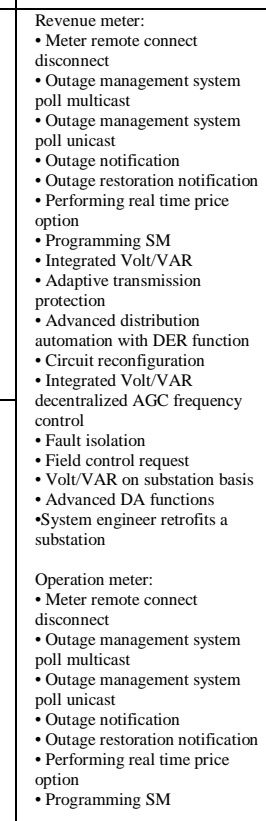 } & \\
\hline & $\begin{array}{l}\text { IEC 62056-3- } \\
1: 2013\end{array}$ & 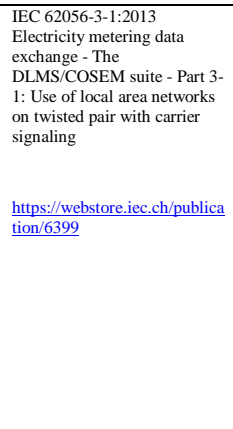 & & & & & & & & 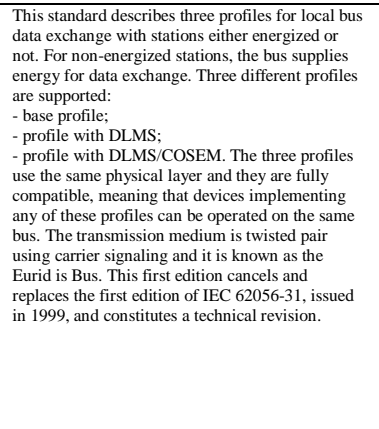 & 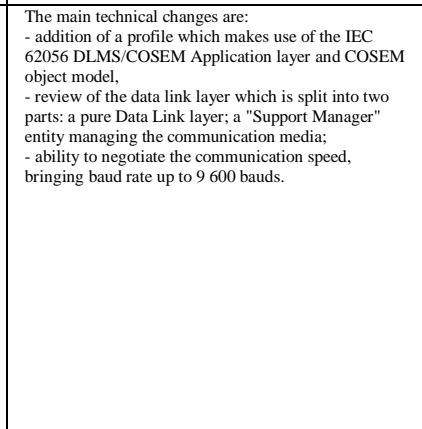 & & & \\
\hline
\end{tabular}




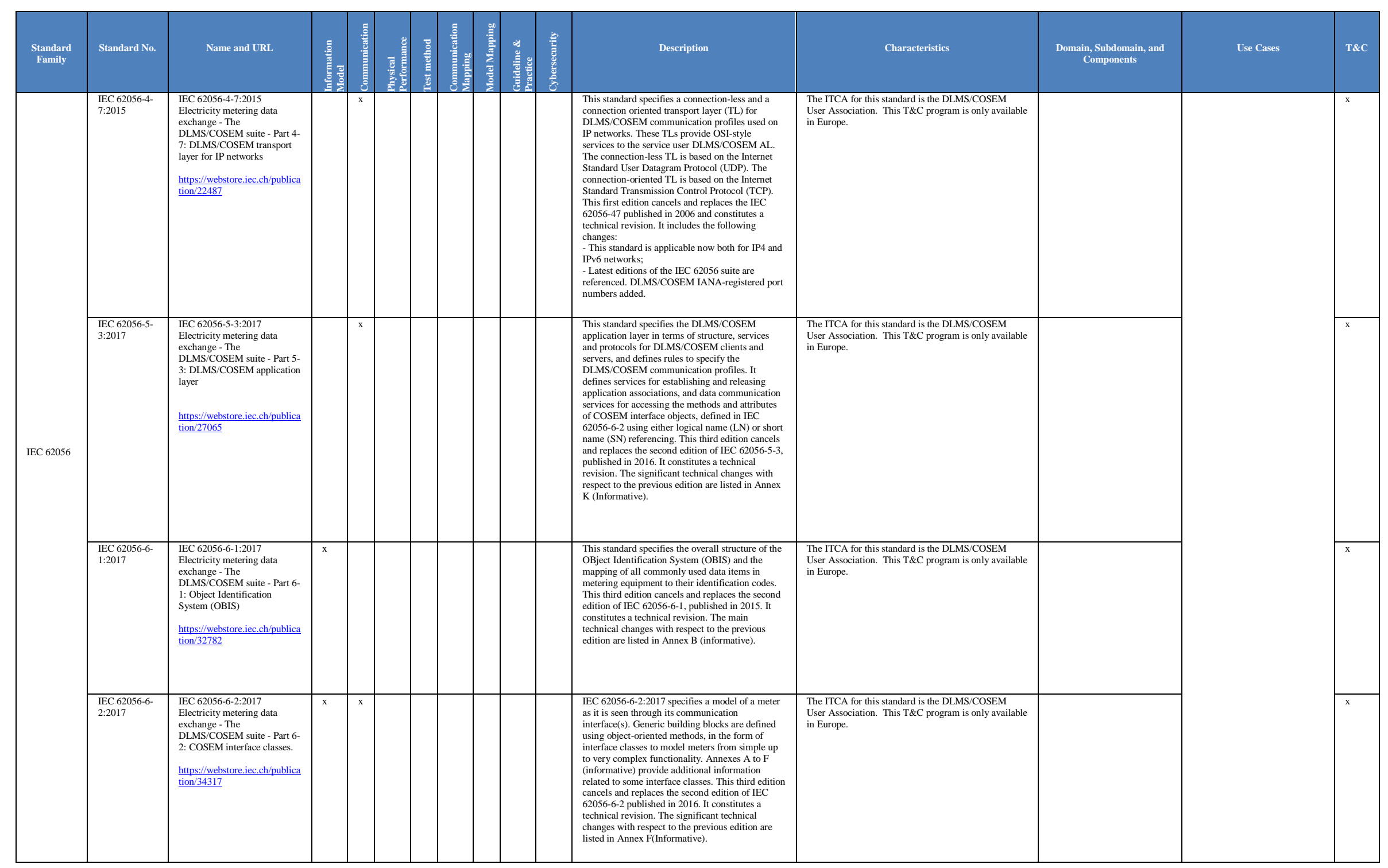




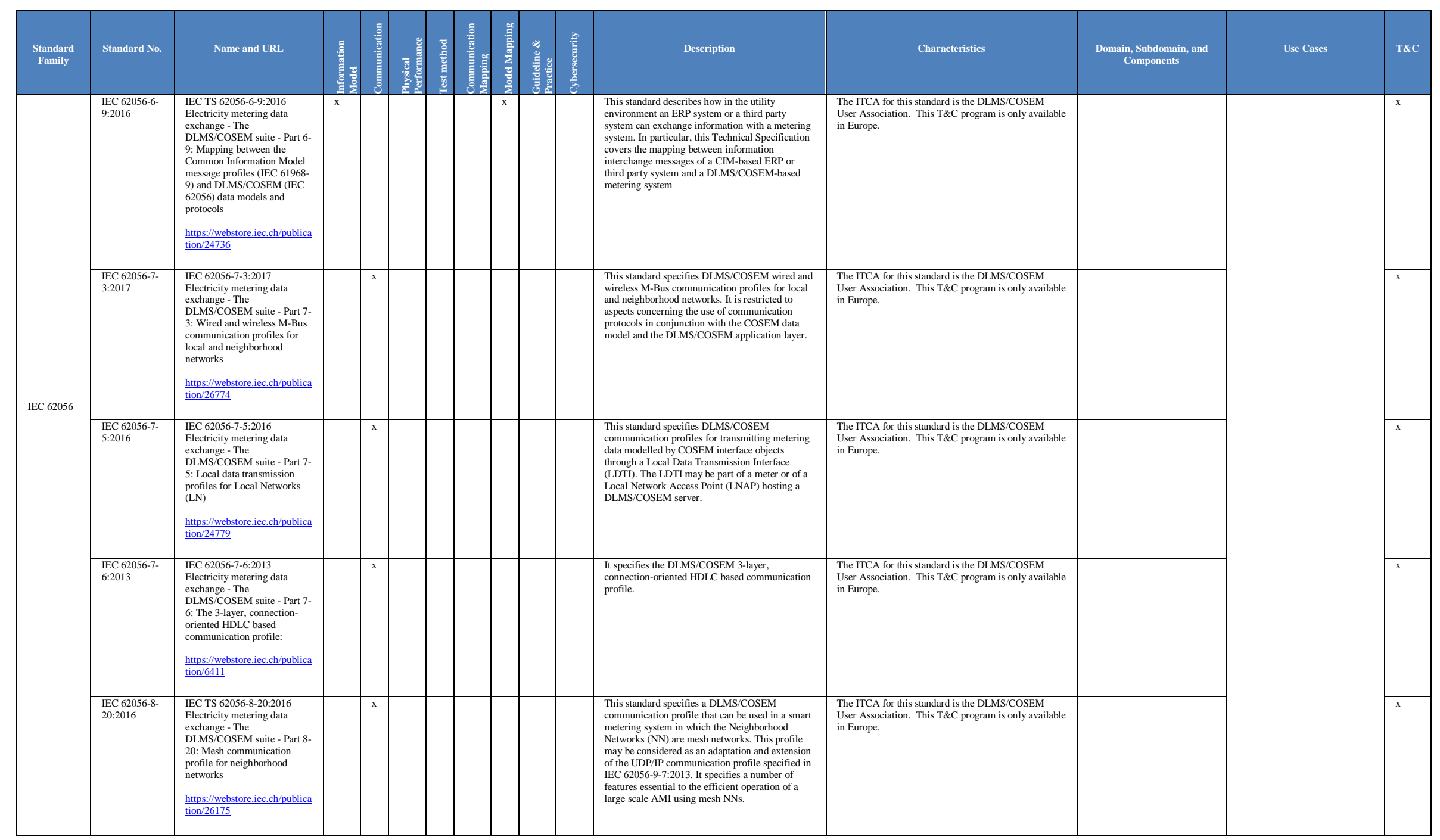




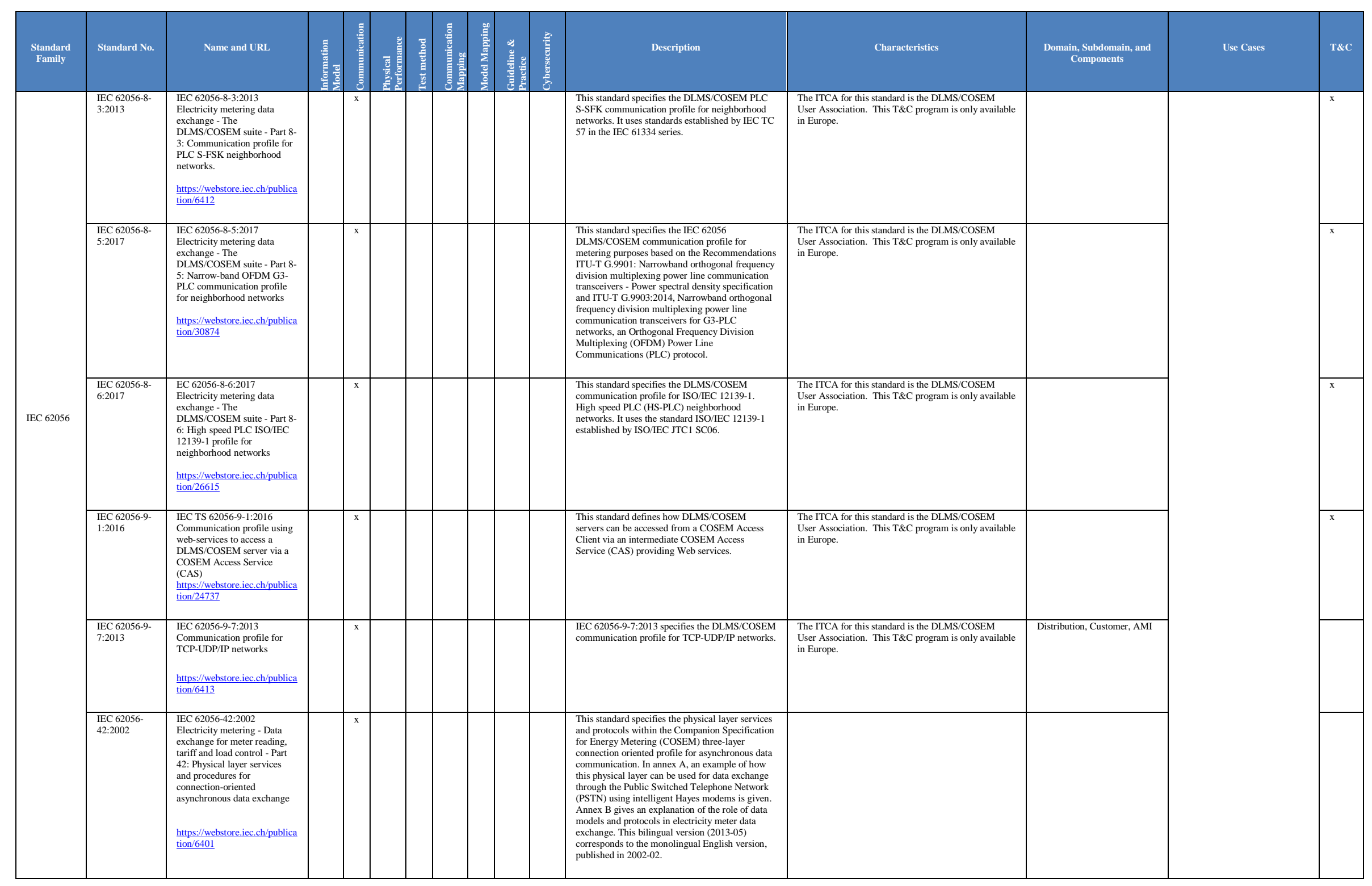




\begin{tabular}{|c|c|c|c|c|c|c|c|c|c|c|c|c|c|}
\hline $\begin{array}{c}\text { Standard } \\
\text { Family }\end{array}$ & Standard No. & Name and URL & & ฮ & 竧 & & 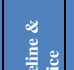 & 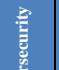 & Description & Characteristics & $\begin{array}{l}\text { Domain, Subdomain, and } \\
\text { Commonents }\end{array}$ & Use Cases & $\mathrm{T \& C}$ \\
\hline IEC 62056 & $\begin{array}{l}\text { IFC 62056- } \\
46: 20202\end{array}$ & 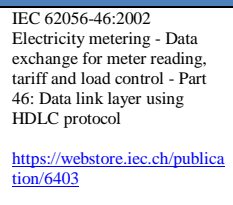 & x & & & & & & 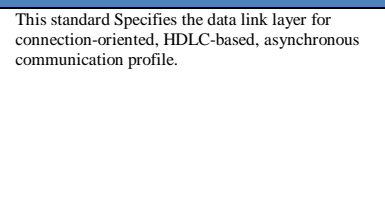 & & Distribution, Customer, AMI & & \\
\hline \multirow{3}{*}{ IEC 62282} & $\begin{array}{l}\text { IEC G2282- } \\
1: 2013\end{array}$ & $\begin{array}{l}\text { IEC TS 62282-1:2013 } \\
\text { Fuel cell technologies - Part } \\
\text { 1: Terminology } \\
\text { https://webstore.eec.ch/publica } \\
\text { tion/6751 }\end{array}$ & & & & & $x$ & & 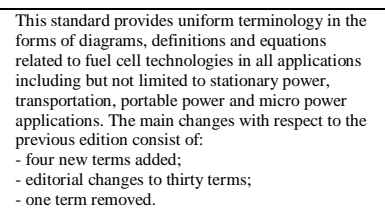 & & - DER Energy storage & \multirow[t]{3}{*}{ 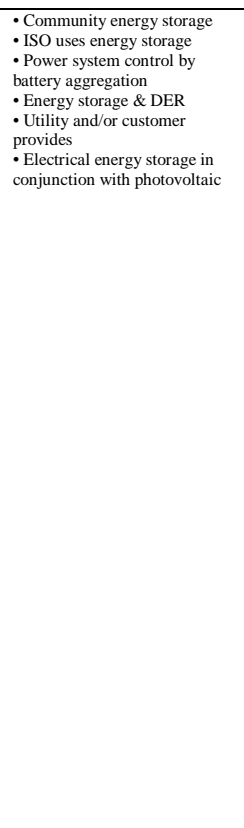 } & \\
\hline & $\begin{array}{l}\text { IEC G28282- } \\
2: 2012\end{array}$ & $\begin{array}{l}\text { IEC 62282-2:2012 } \\
\text { Fuel cell technologies - Part } \\
\text { 2: Ful cell modules } \\
\text { hittps://webstore.eec.ch/publica } \\
\text { tion/6752 }\end{array}$ & & $\mathrm{x}$ & & & & & 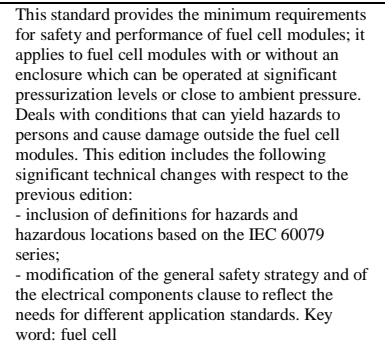 & & & & \\
\hline & $\begin{array}{l}\text { IEC 62282-3- } \\
100: 2012\end{array}$ & $\begin{array}{l}\text { IEC 62282-3-100:2012 } \\
\text { Fuel cell technologies - Part } \\
\text { 3-100: Stationaly fuel cell } \\
\text { power systems - Safety } \\
\text { hhtpps//webstore.ece.ch/publica } \\
\text { tion/6753 }\end{array}$ & & & & & $x$ & & 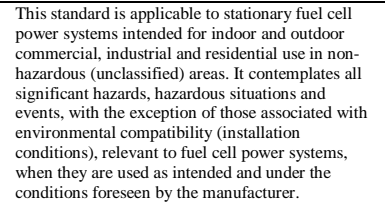 & & & & \\
\hline
\end{tabular}




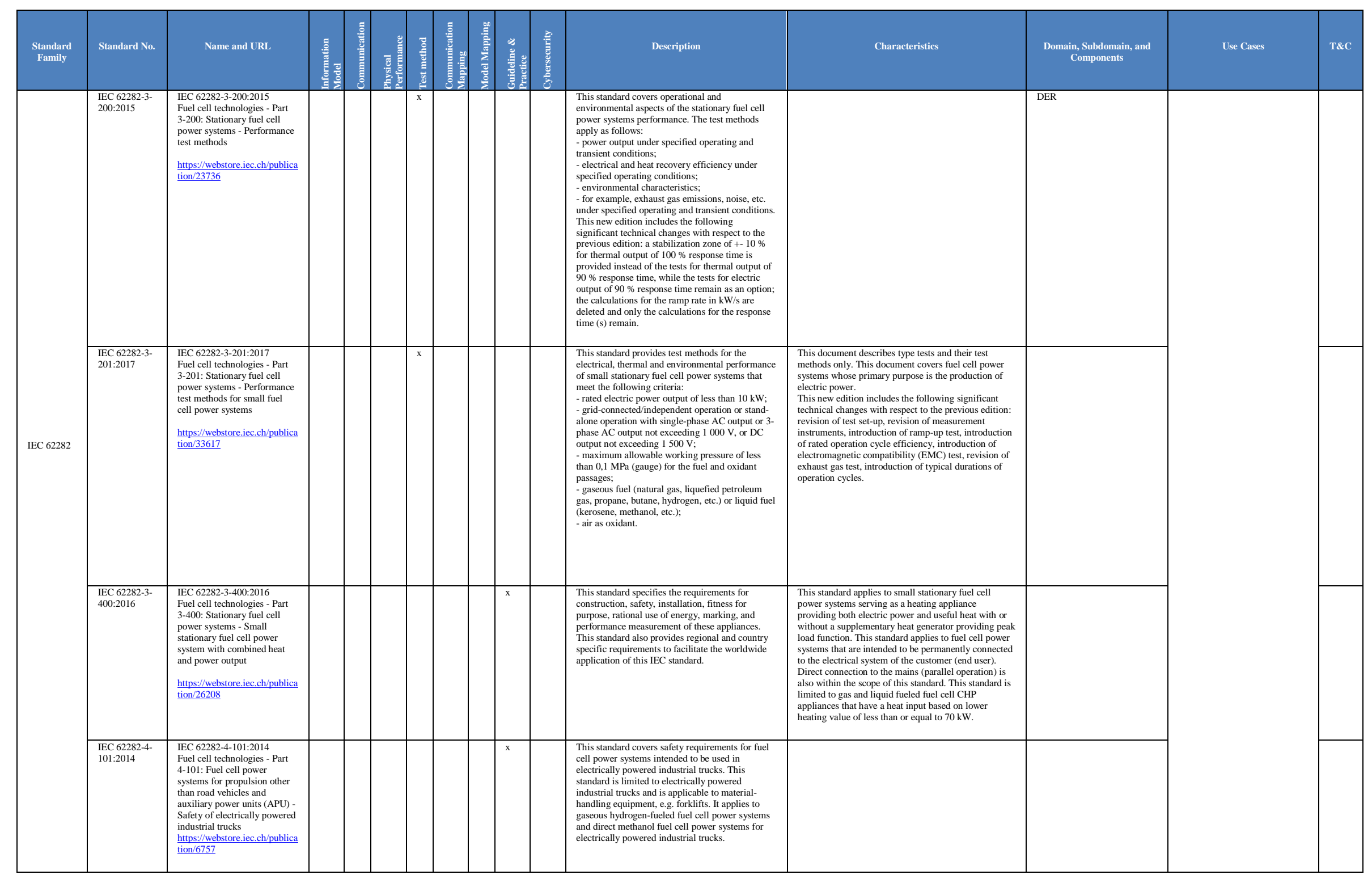




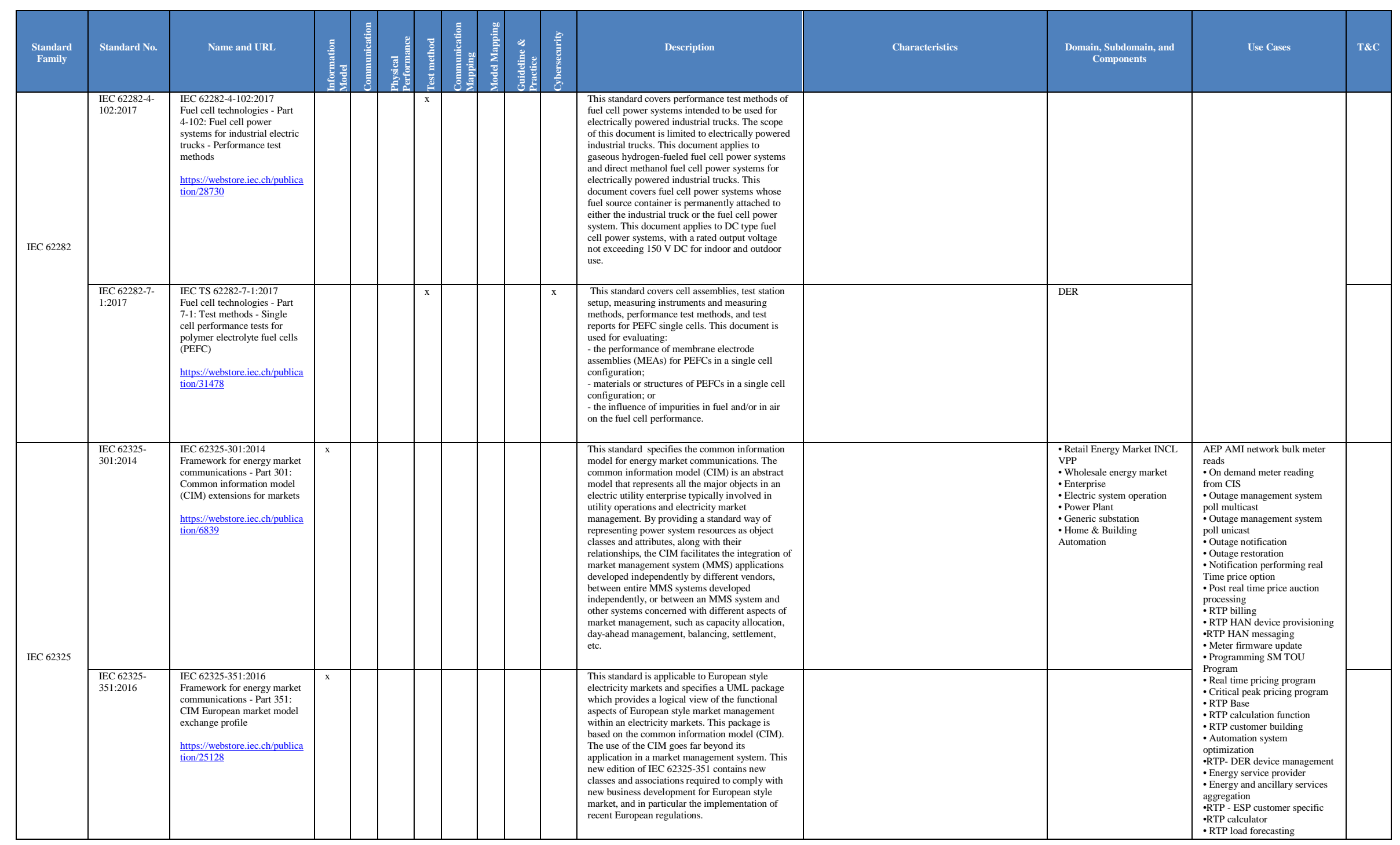




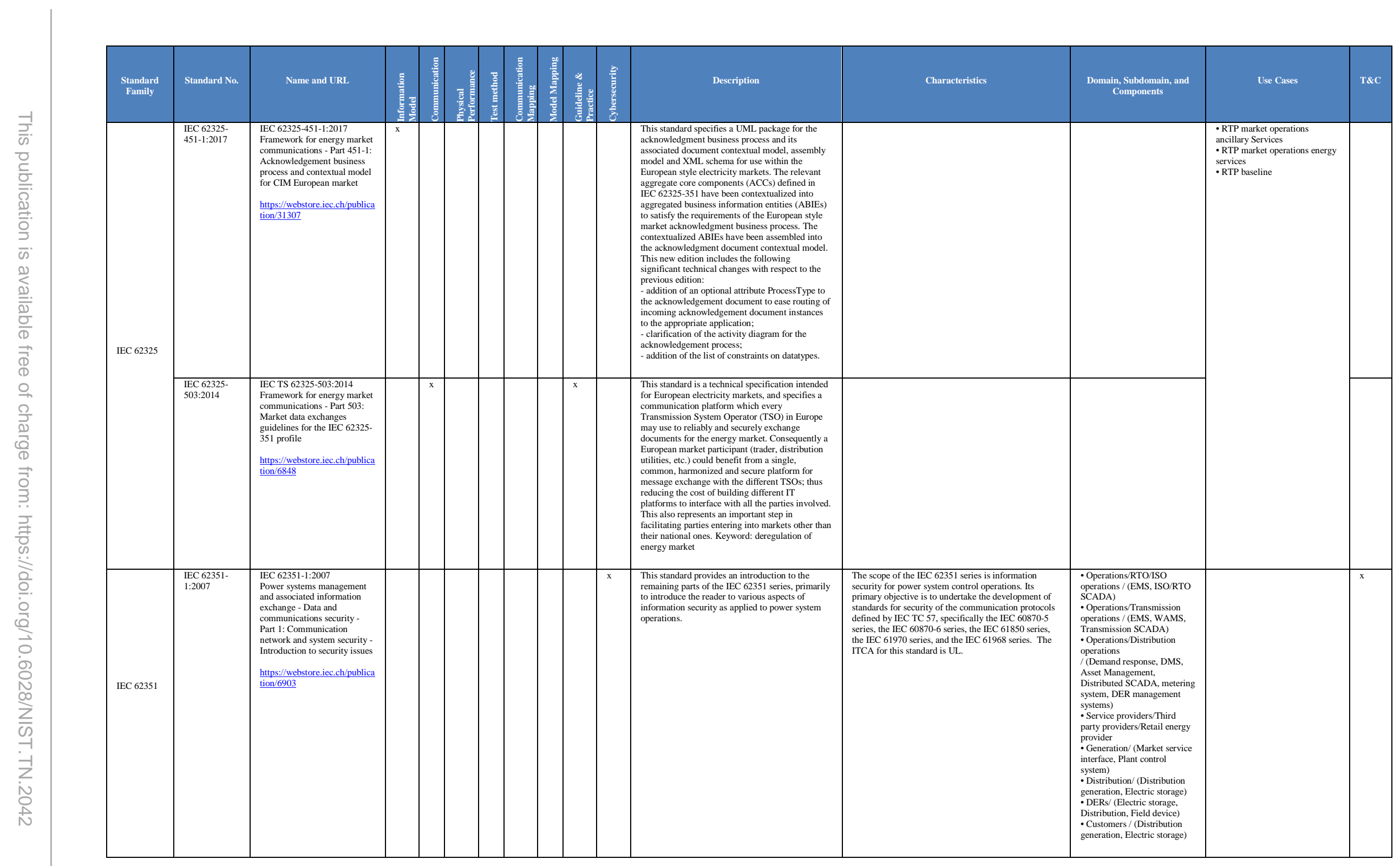




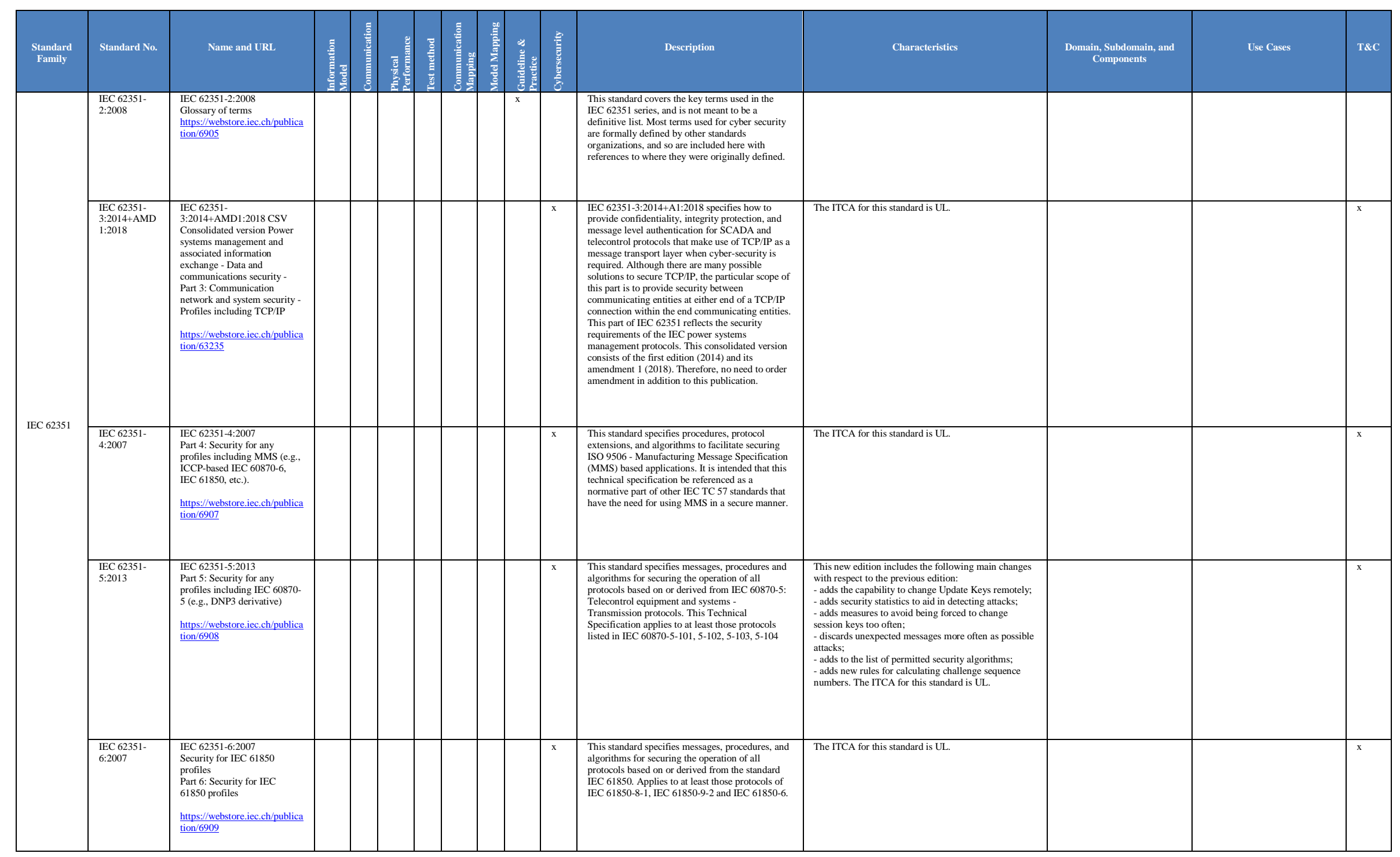




\begin{tabular}{|c|c|c|c|c|c|c|c|c|c|c|c|c|c|}
\hline $\begin{array}{c}\text { Standard } \\
\text { Family }\end{array}$ & Standard No. & Name and URL & & & & 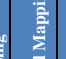 & $\stackrel{\infty}{\infty}$ & 急 & Description & Characteristics & $\begin{array}{l}\text { Domain, Subdomain, and } \\
\text { Commponents }\end{array}$ & Use Cases & T\&C \\
\hline \multirow{5}{*}{ IEC 62351} & $\begin{array}{l}\text { IEC 62351- } \\
7: 2017\end{array}$ & 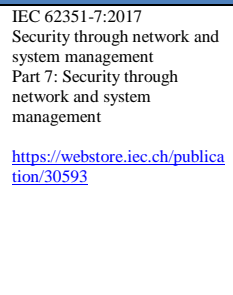 & & & & & & & 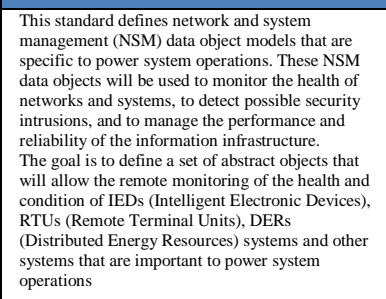 & 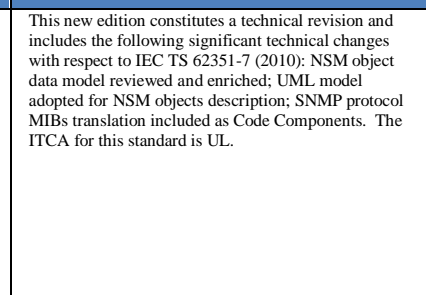 & & & \\
\hline & $\begin{array}{l}\mathrm{IEC} 62351- \\
8: 2011\end{array}$ & $\begin{array}{l}\text { IEC 62351-8:2011 } \\
\text { Power systems management } \\
\text { and associted information } \\
\text { exchange - Data and } \\
\text { communication s security - } \\
\text { Part 8 8 Role-based access } \\
\text { control } \\
\text { hittps://webstore.eec.ch/publica } \\
\text { tion/6911 }\end{array}$ & & & & & & 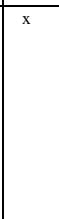 & 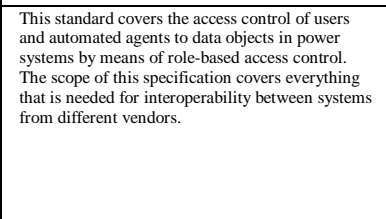 & \begin{tabular}{|l|} 
The ITCA for this standard is UL. \\
\end{tabular} & & & $x$ \\
\hline & $\begin{array}{l}\mathrm{IEC6} 62351- \\
9: 2017\end{array}$ & 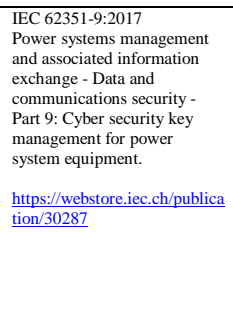 & & & & & & $x$ & 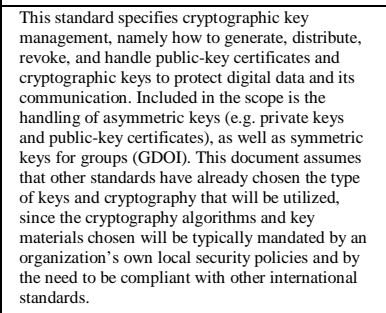 & 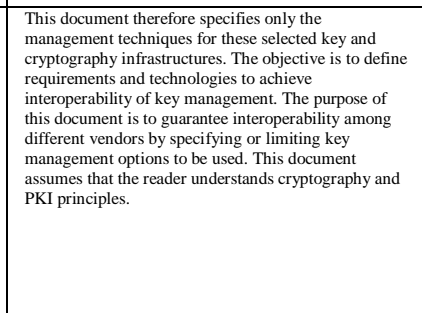 & & & \\
\hline & $\begin{array}{l}\frac{\mathrm{IIEC} 62351-}{10: 2012} \\
\text { 1020 }\end{array}$ & $\begin{array}{l}\text { IEC TR 62351-10:2012 } \\
\text { Power systems management } \\
\text { and associted information } \\
\text { exchange - Data and } \\
\text { communication security - } \\
\text { Part 10: Security architecture } \\
\text { guidelines. } \\
\text { hittps///webstore.eec.ch/phublica } \\
\text { tion/6904 }\end{array}$ & & & & & $\mathrm{x}$ & \begin{tabular}{|l|}
$x$ \\
\end{tabular} & 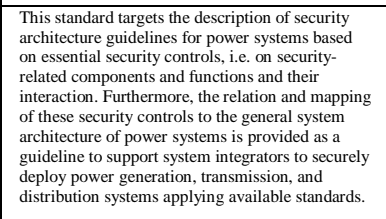 & & & & \\
\hline & 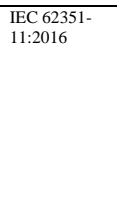 & $\begin{array}{l}\text { IEC 62351-11:2016 } \\
\text { Power systems management } \\
\text { and associted information } \\
\text { exchange - Data and } \\
\text { communication s security- } \\
\text { Part 11: Security for XML } \\
\text { documents. } \\
\text { hittps///webstore.eec.ch/publica } \\
\text { tion/25948 }\end{array}$ & & & & & & \begin{tabular}{|l|}
$x$ \\
\end{tabular} & 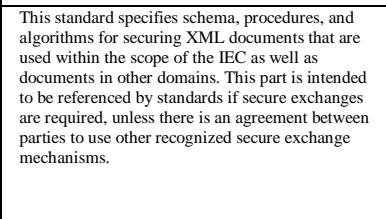 & 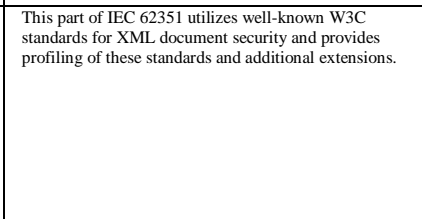 & & & \\
\hline
\end{tabular}




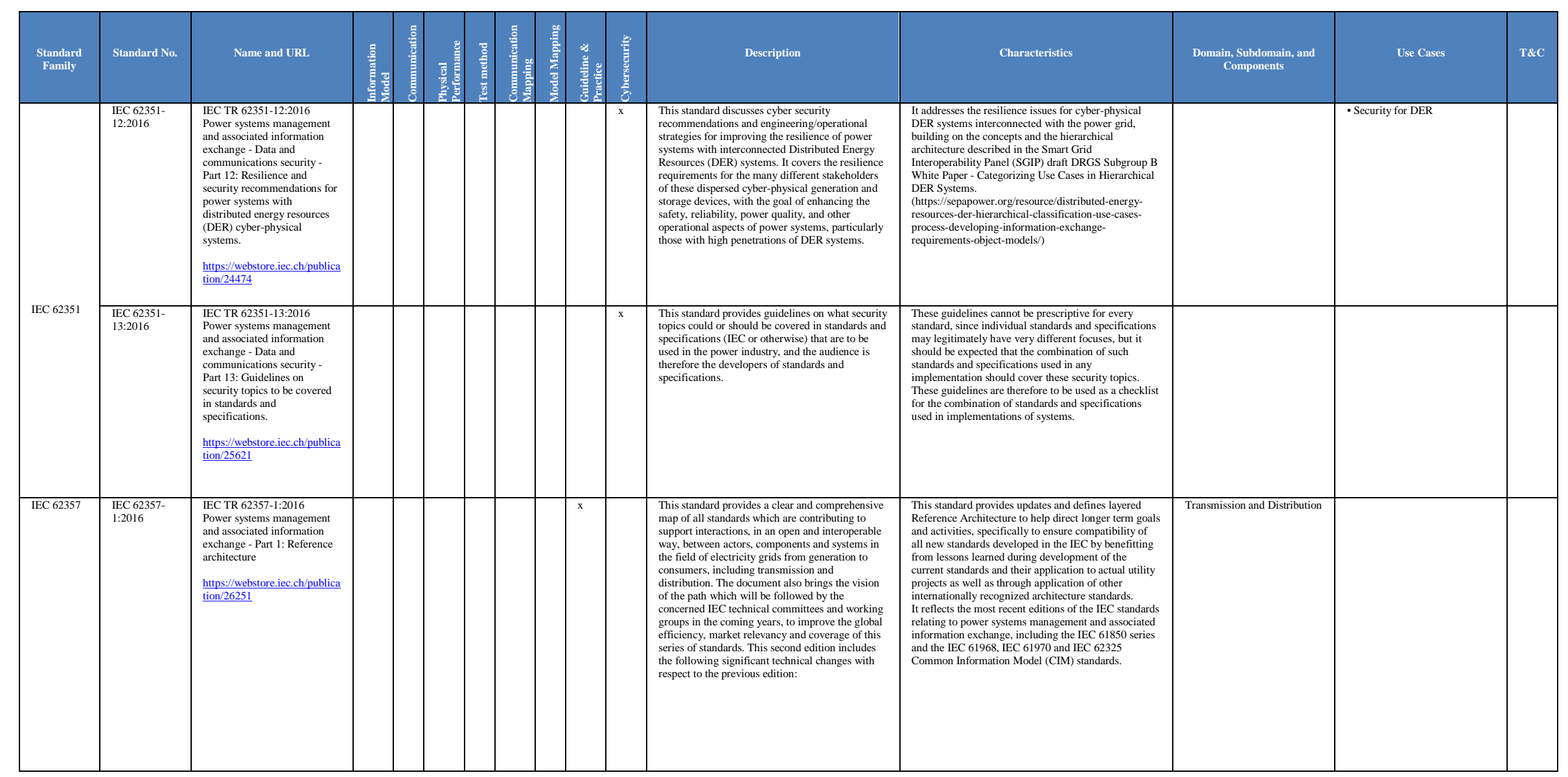




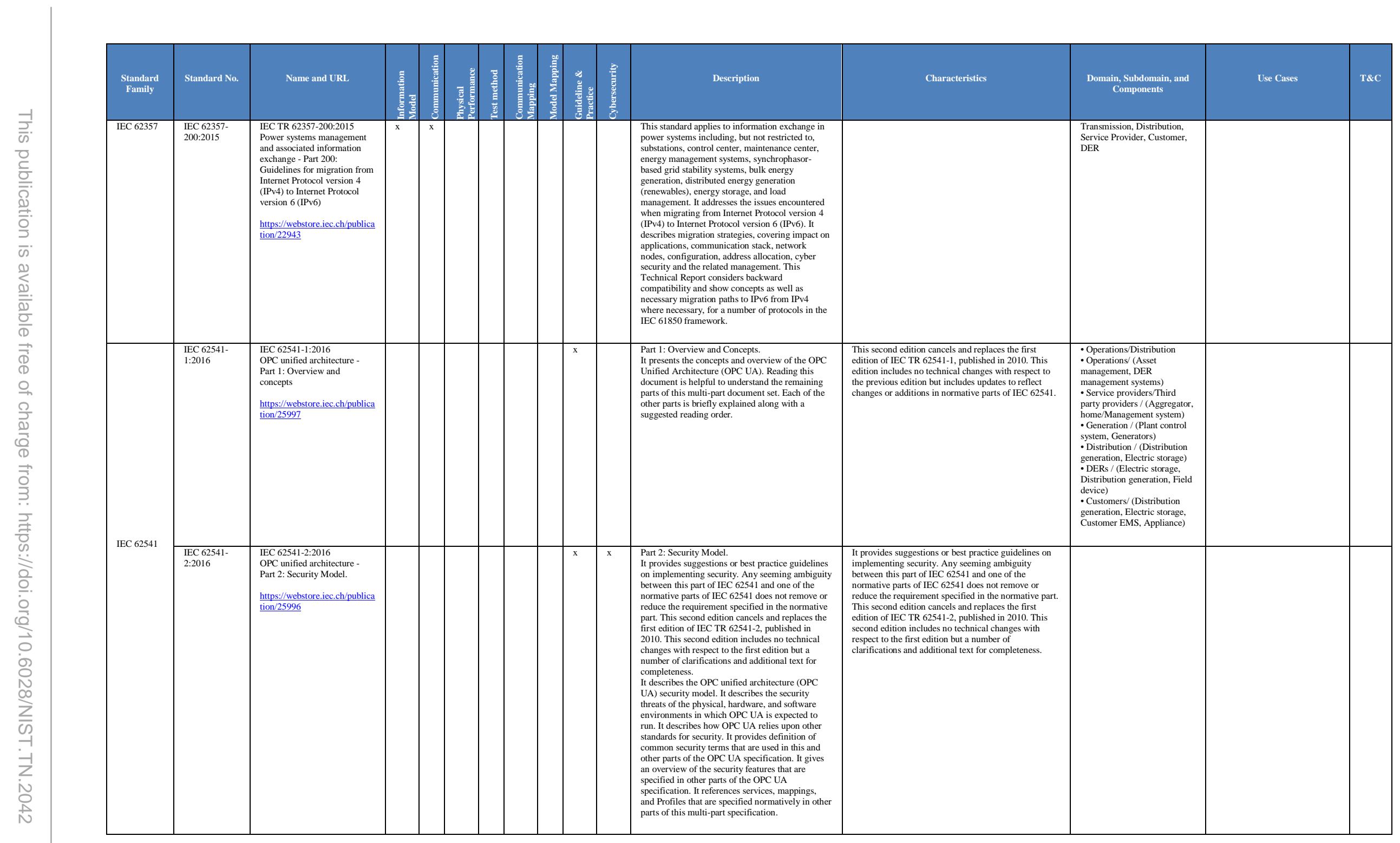




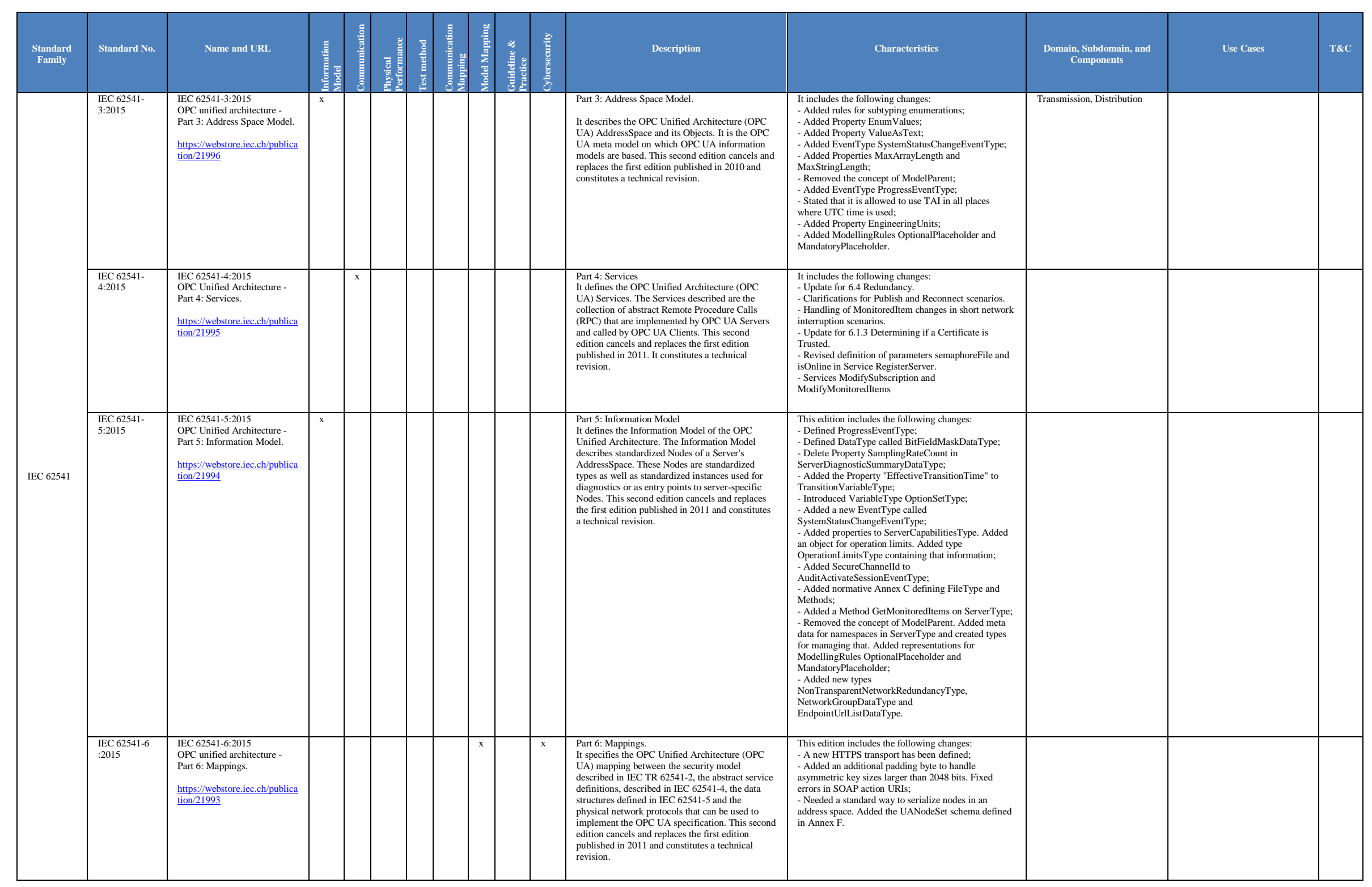




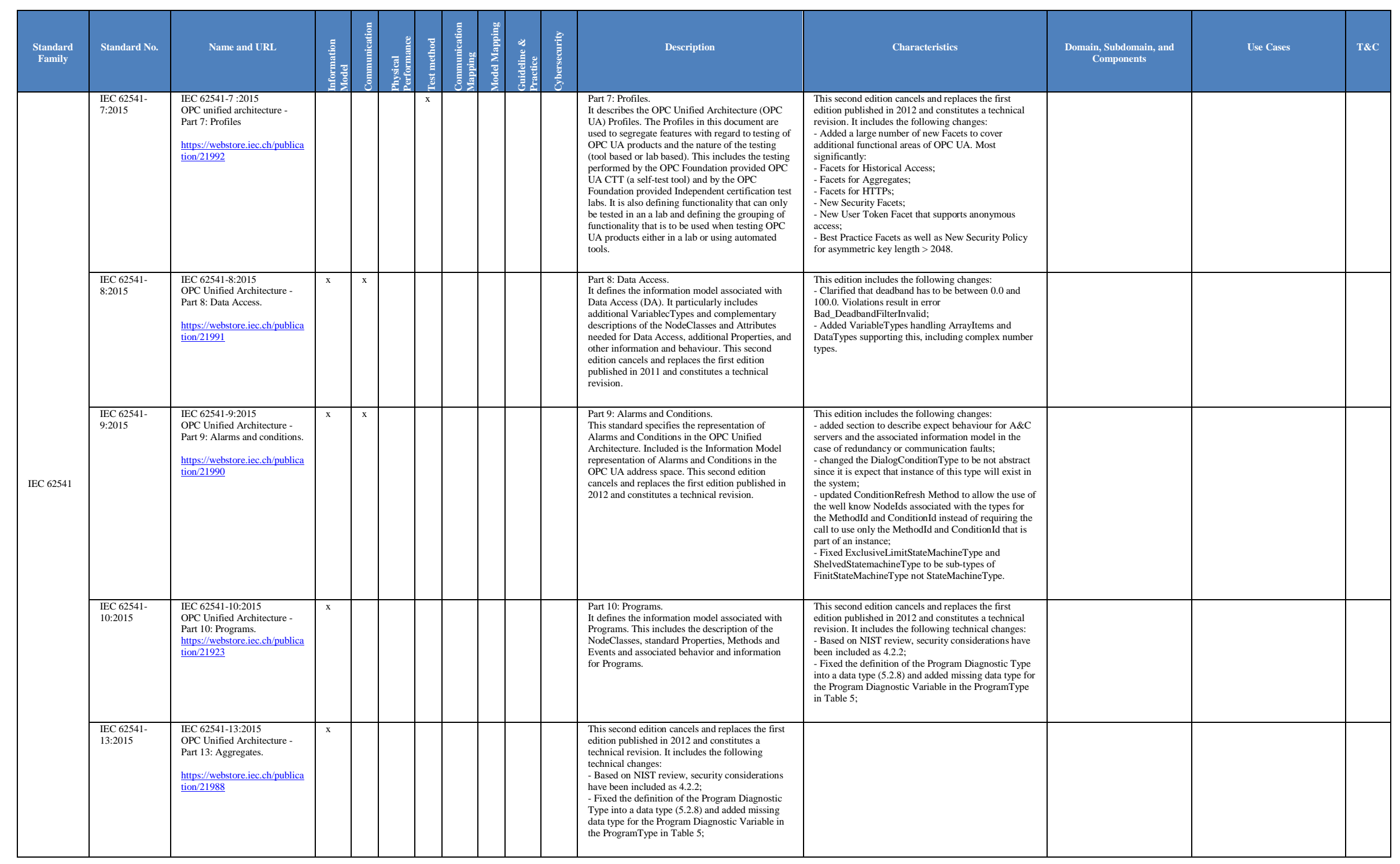




\begin{tabular}{|c|c|c|c|c|c|c|c|c|c|c|c|c|c|c|}
\hline 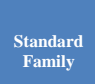 & Standard No. & Name and URL & & & 鼌 & & & 童 & 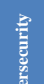 & Description & Characterisitics & $\begin{array}{l}\text { Domain, Subdomain, and } \\
\text { Components }\end{array}$ & Use Cases & $\mathrm{T} \& \mathrm{C}$ \\
\hline IEC 62541 & $\begin{array}{l}\text { IEC } 62541- \\
100: 2015\end{array}$ & $\begin{array}{l}\text { IEC 62541-100:2015 } \\
\text { OOC Unified Architecture - } \\
\text { Part 100: Device Interface. } \\
\text { https://webstore.eec.ch/publica } \\
\text { tion/21987 }\end{array}$ & & & & & & & & 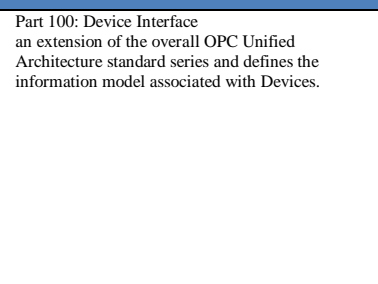 & 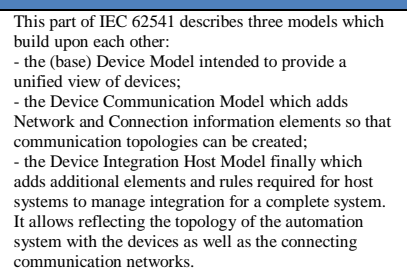 & & & \\
\hline \multirow{3}{*}{ IEC 62689} & 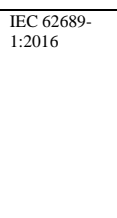 & 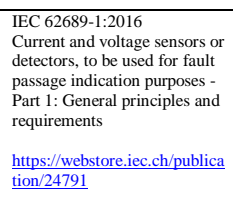 & & & & & & $\mathrm{x}$ & & 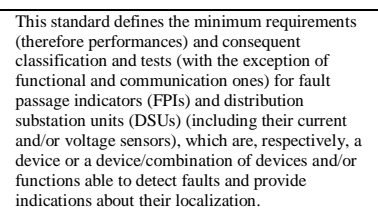 & & \begin{tabular}{|l|l|} 
Transmission, Distribution \\
\end{tabular} & & \\
\hline & $\begin{array}{l}\text { IIEC 62689- } \\
2: 2016\end{array}$ & 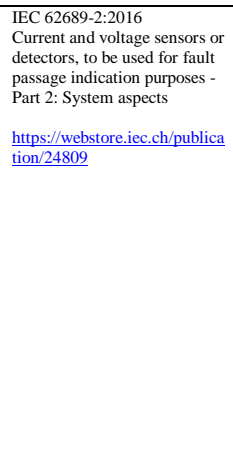 & & & & & & & & 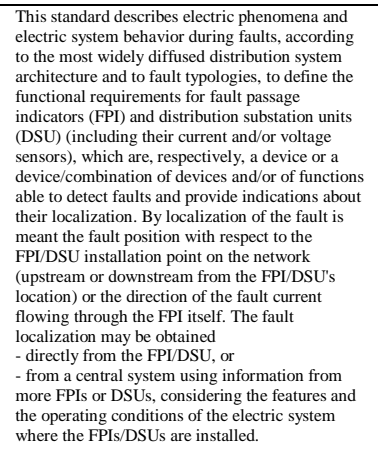 & 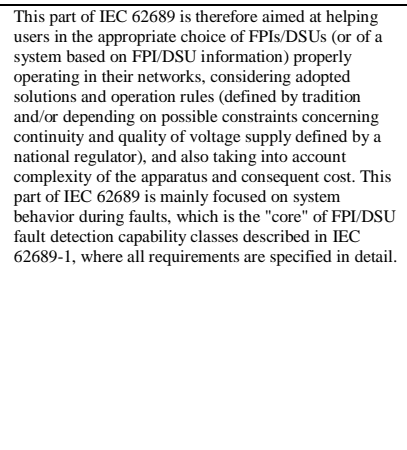 & \begin{tabular}{|c|} 
Transmission, Distribution \\
\end{tabular} & & \\
\hline & $\begin{array}{l}\text { IEC G26899- } \\
100: 2016\end{array}$ & 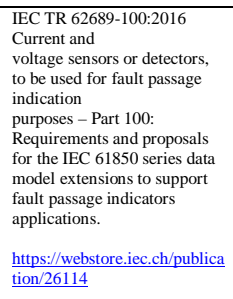 & $\mathrm{x}$ & & & & $\mathrm{x}$ & & & 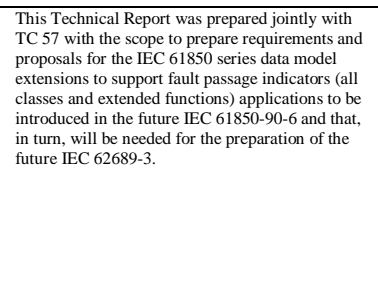 & & \begin{tabular}{|l|l|l} 
Transmisision, Distribution \\
\end{tabular} & & \\
\hline
\end{tabular}




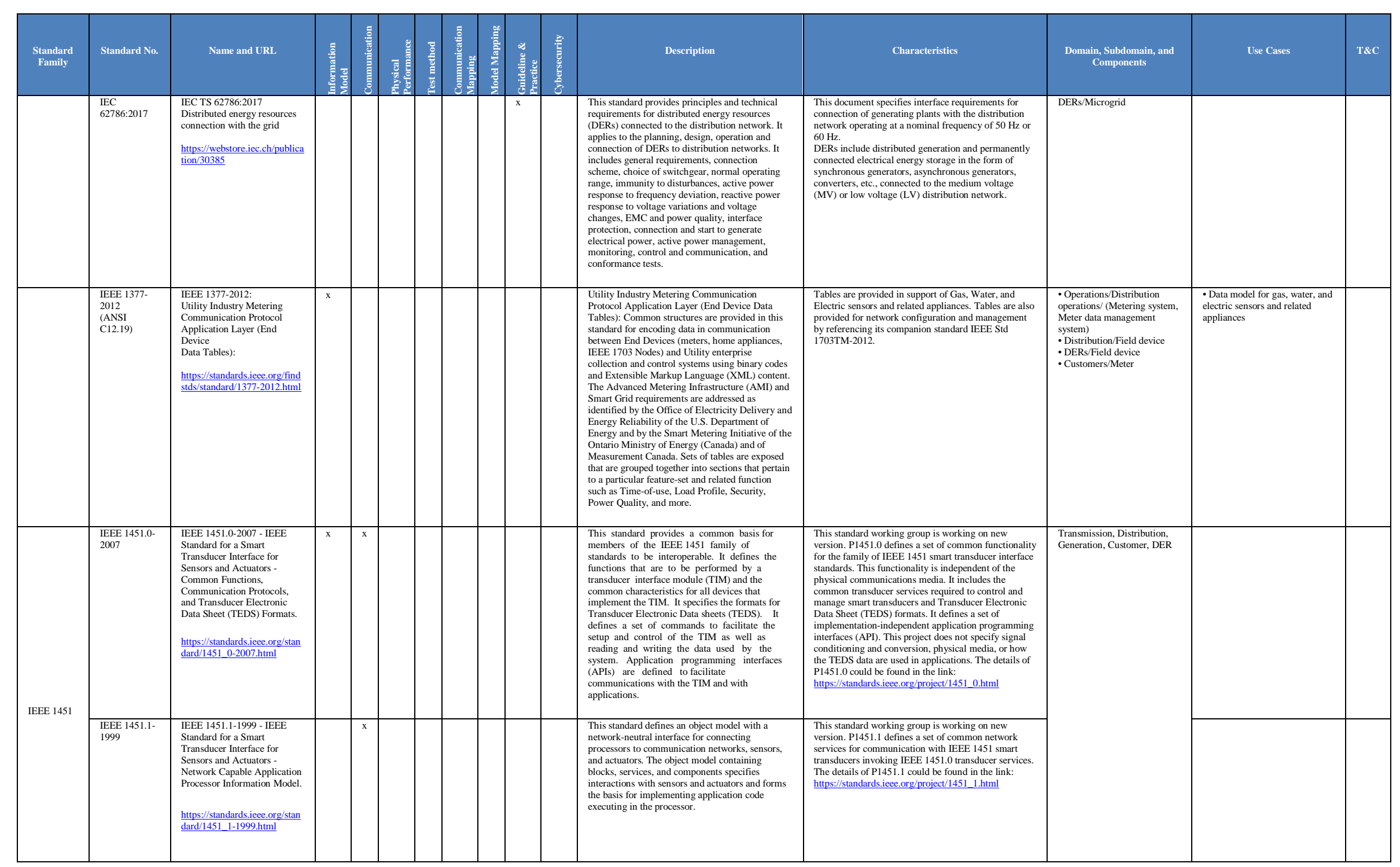




\begin{tabular}{|c|c|c|c|c|c|c|c|c|c|c|c|c|}
\hline $\begin{array}{c}\text { Standardd } \\
\text { Family }\end{array}$ & Standard No. & Name and URL & & & & 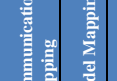 & & Description & Characterisitics & $\begin{array}{l}\text { Domain, Subdomain, and } \\
\text { Components }\end{array}$ & Use Cases & T\&C \\
\hline \multirow[t]{2}{*}{ IEEE 1451} & $\begin{array}{l}\text { IEEE 1451.4- } \\
2004\end{array}$ & 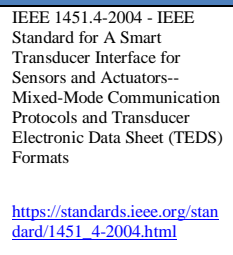 & & & & & & 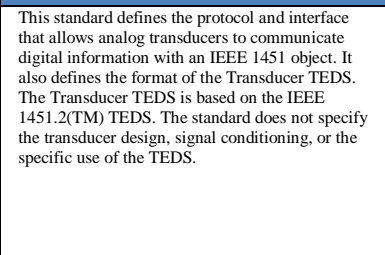 & 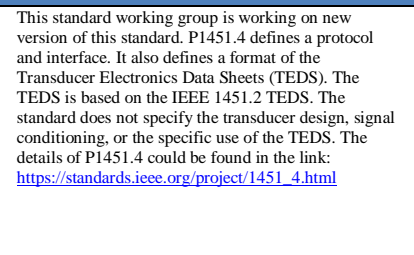 & & & \\
\hline & $\begin{array}{l}l_{\text {IIEEE }} \\
2007\end{array}$ & 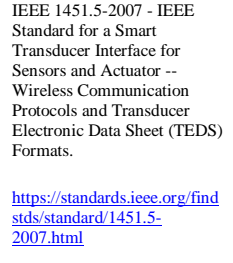 & & & & & & 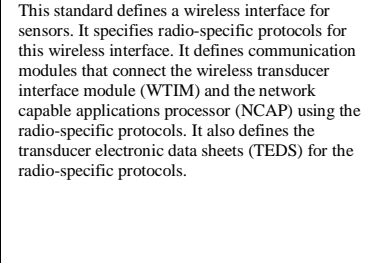 & 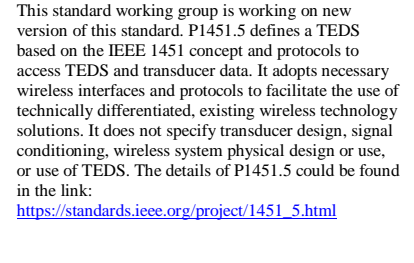 & & & \\
\hline \multirow[b]{2}{*}{ IEEE 1547} & $\begin{array}{l}\text { IEEE 1547- } \\
2018\end{array}$ & 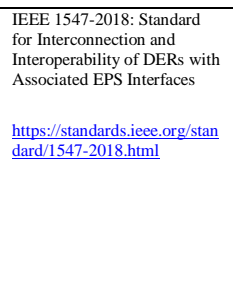 & & $x$ & & & & 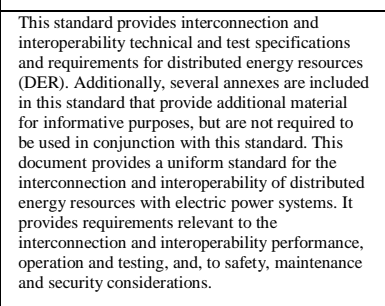 & 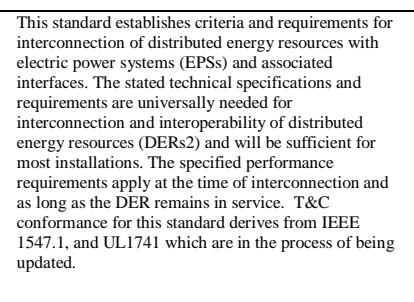 & \begin{tabular}{|l} 
Transmission, Distribution, \\
Customer, DER
\end{tabular} & & $y$ \\
\hline & $\begin{array}{l}\text { IFEE 1547.1- } \\
{ }_{2005}\end{array}$ & 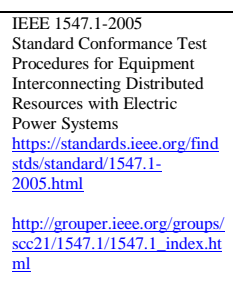 & & & $\mathrm{x}$ & & & 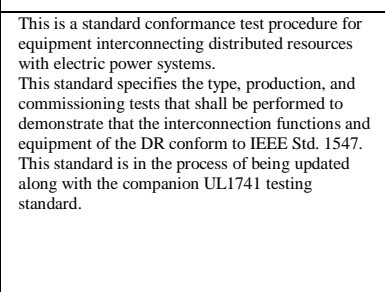 & The planned ITCA for this standard is I LAP. & \begin{tabular}{|l}
$\begin{array}{l}\text { Transmission, Distribution, } \\
\text { Customer, DERR }\end{array}$ \\
\end{tabular} & & $\mathrm{p}$ \\
\hline
\end{tabular}




\begin{tabular}{|c|c|c|c|c|c|c|c|c|c|c|c|c|c|c|}
\hline $\begin{array}{c}\text { Standard } \\
\text { Family }\end{array}$ & Standard No. & Name and URL & & & & & & & 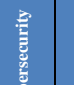 & Description & Characteristics & $\begin{array}{l}\text { Domain, Subdomain, and } \\
\text { Components }\end{array}$ & Use Cases & Tा\& \\
\hline \multirow{4}{*}{ IEEE 1547} & $\begin{array}{l}\text { IEEE 1547.2- } \\
2008\end{array}$ & 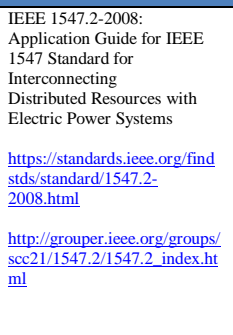 & & & & & & & & 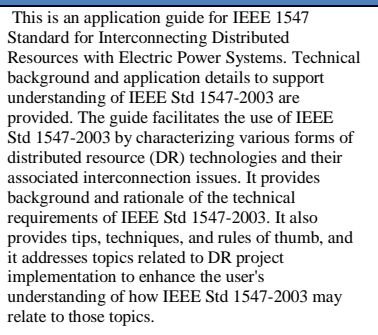 & 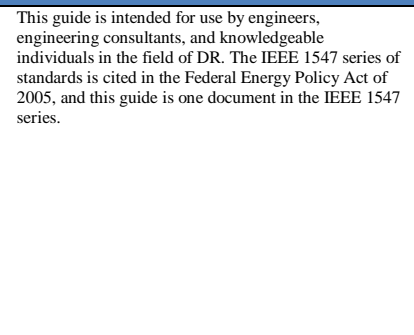 & \begin{tabular}{|l} 
Transmission, Distribution, \\
Customer, DER
\end{tabular} & & \\
\hline & $\begin{array}{l}\text { IEEE 1547.3- } \\
2007\end{array}$ & 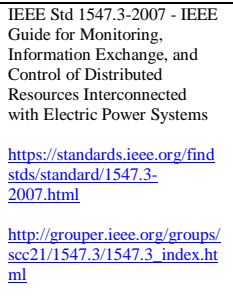 & $\mathrm{x}$ & & & & & & & 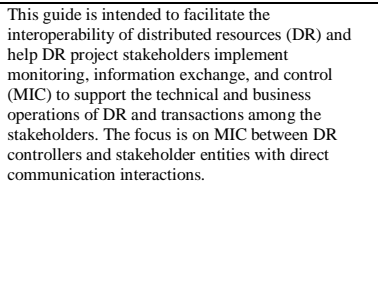 & 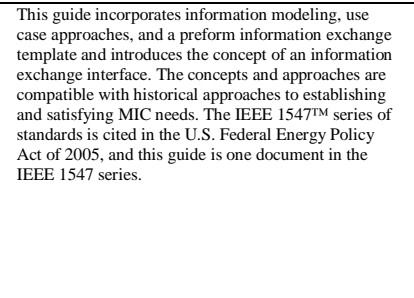 & \begin{tabular}{|l|} 
Transmisssion, Distribution, \\
Customer, DER
\end{tabular} & & \\
\hline & $\begin{array}{l}\text { IEEE 1547.4- } \\
2011\end{array}$ & 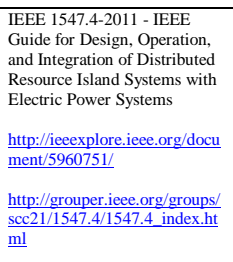 & & & & & & $\mathrm{x}$ & & 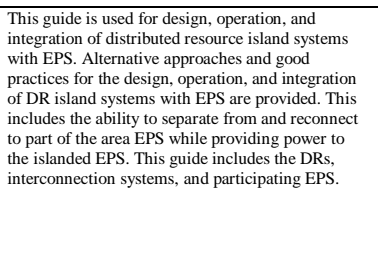 & 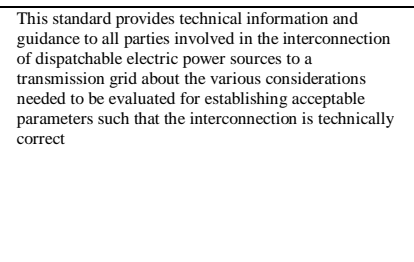 & \begin{tabular}{|l} 
Transmission, Distribution, \\
Customer, DER
\end{tabular} & & \\
\hline & $\begin{array}{l}\mathrm{IEEE} \\
\text { 1547.6:2011 }\end{array}$ & 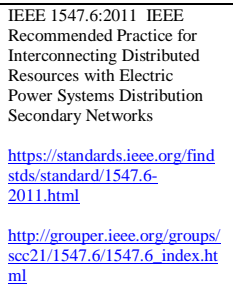 & & & & & & $x$ & & 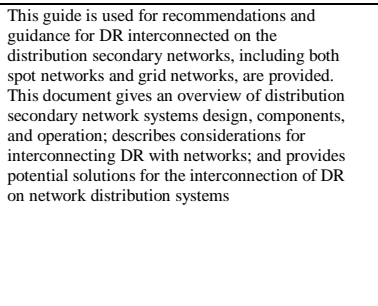 & 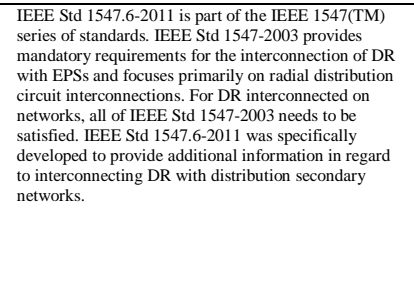 & \begin{tabular}{|l|} 
Transmisssion, Distribution, \\
Customer, DER
\end{tabular} & & \\
\hline
\end{tabular}




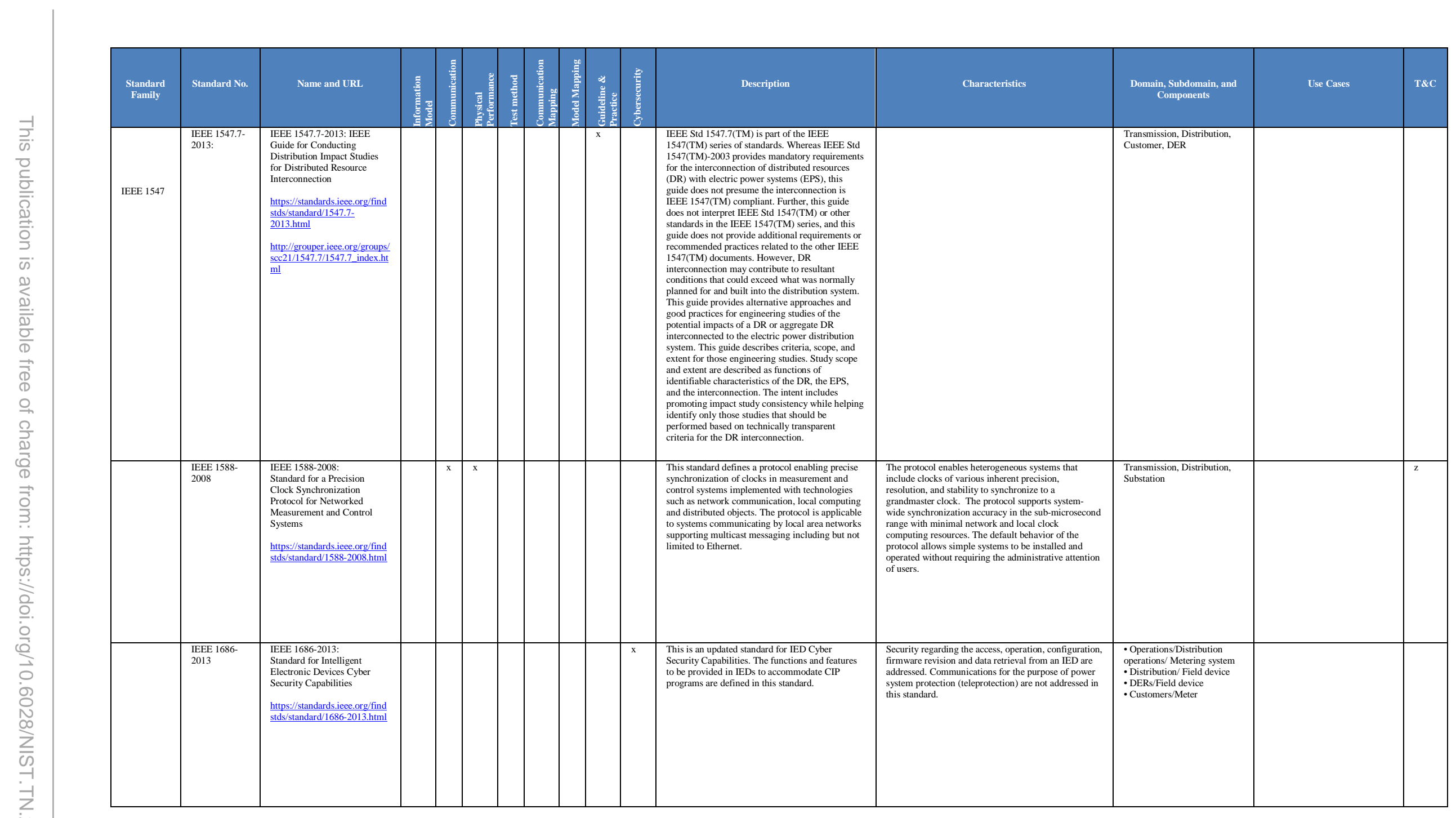




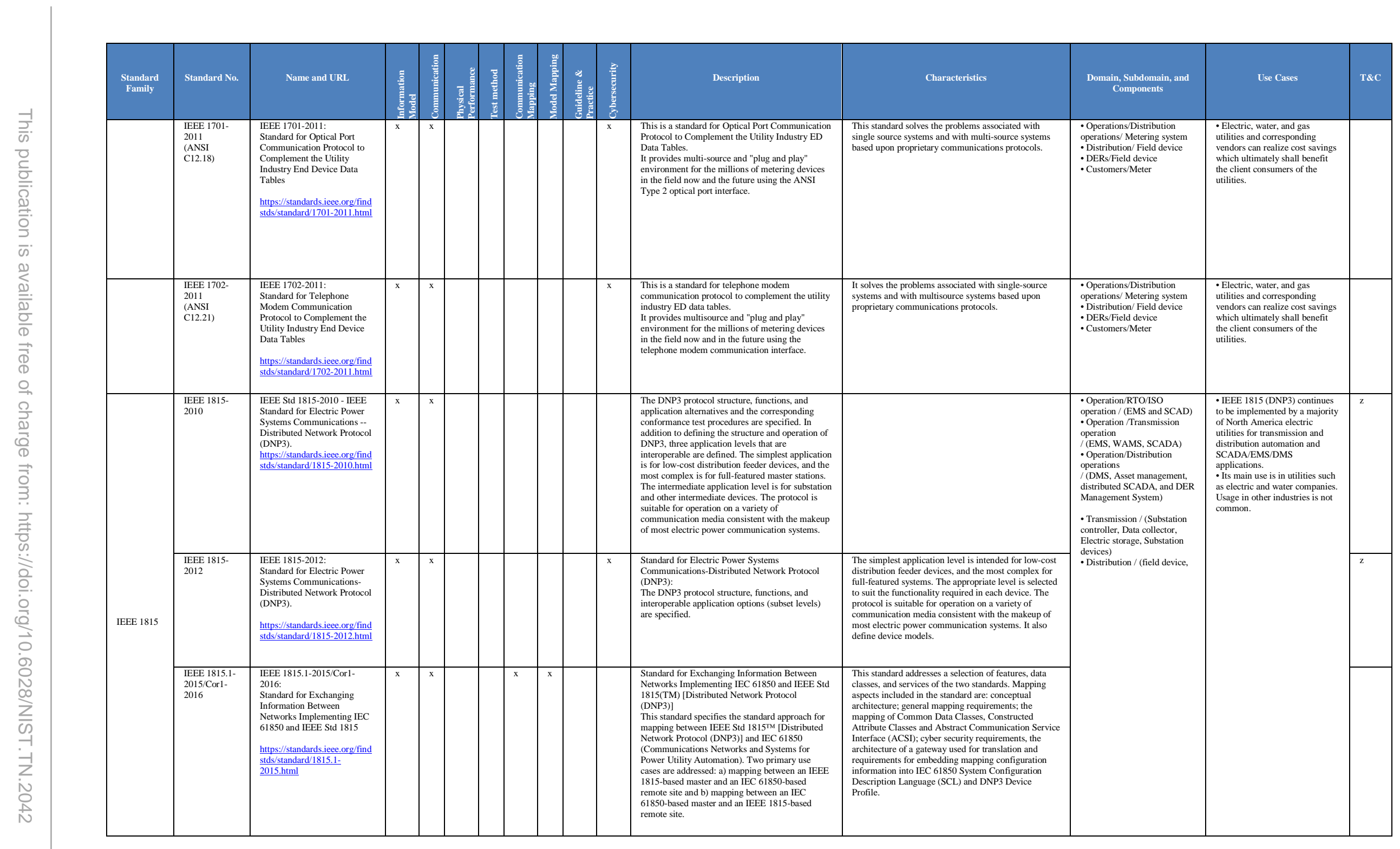




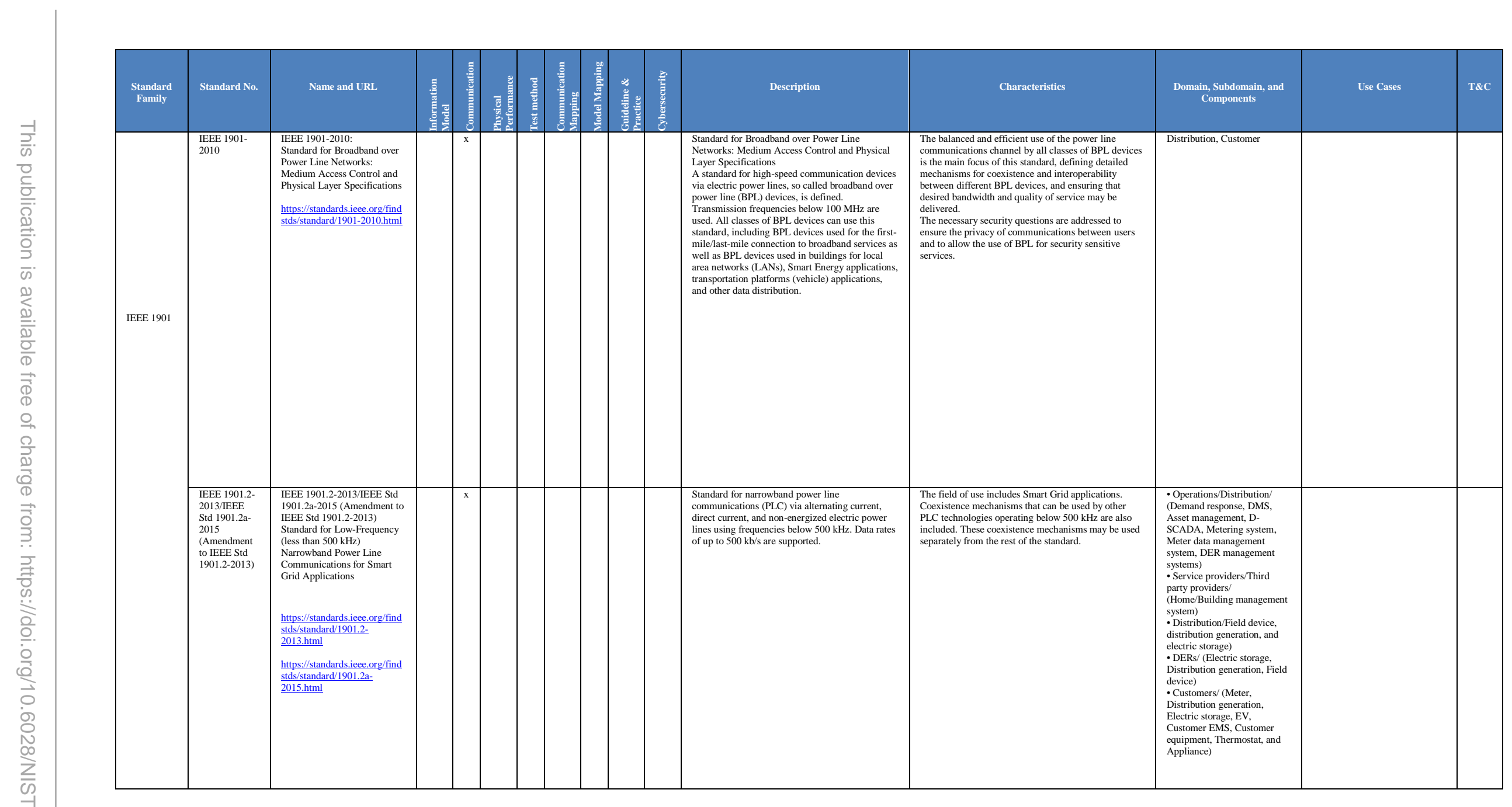




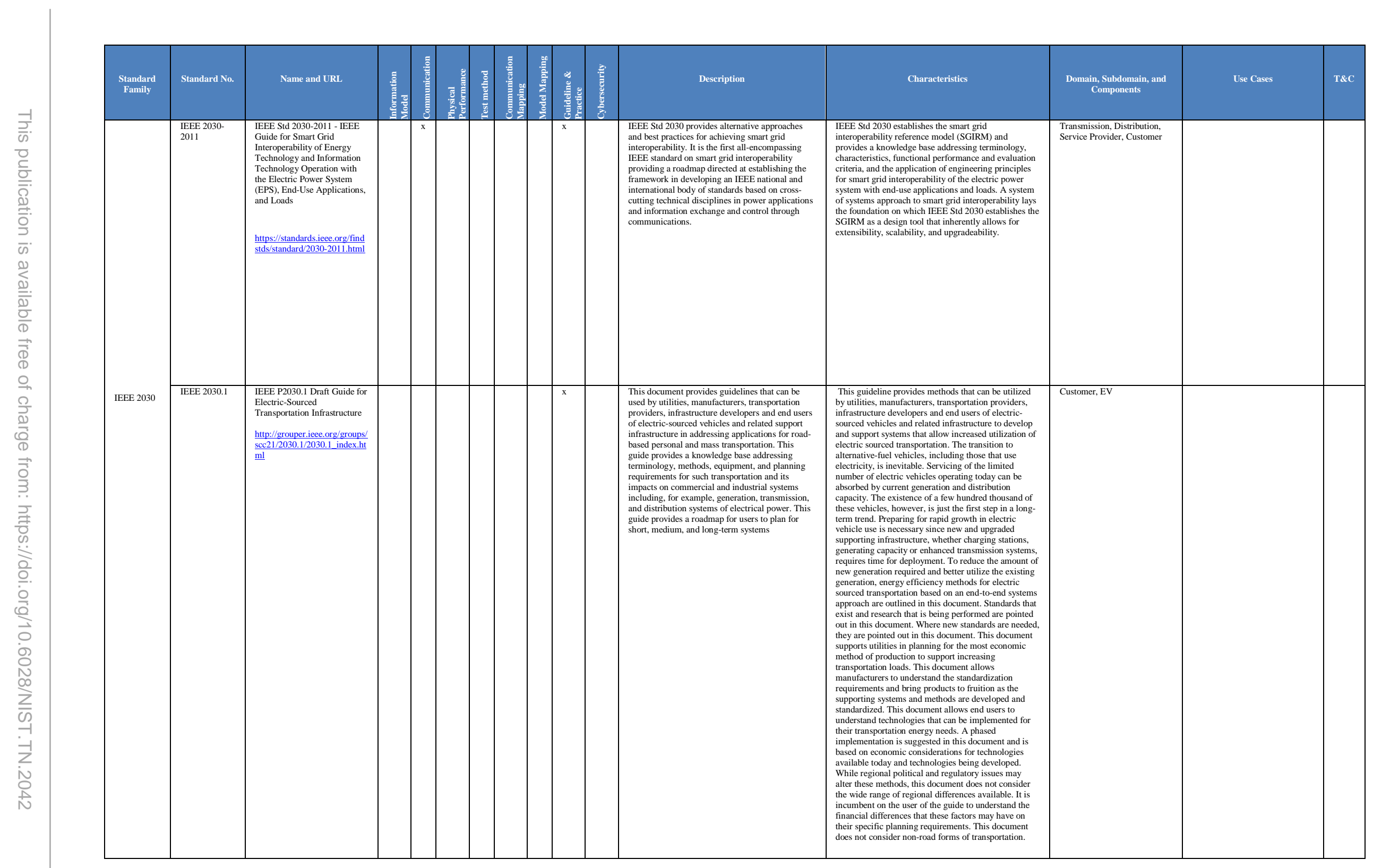




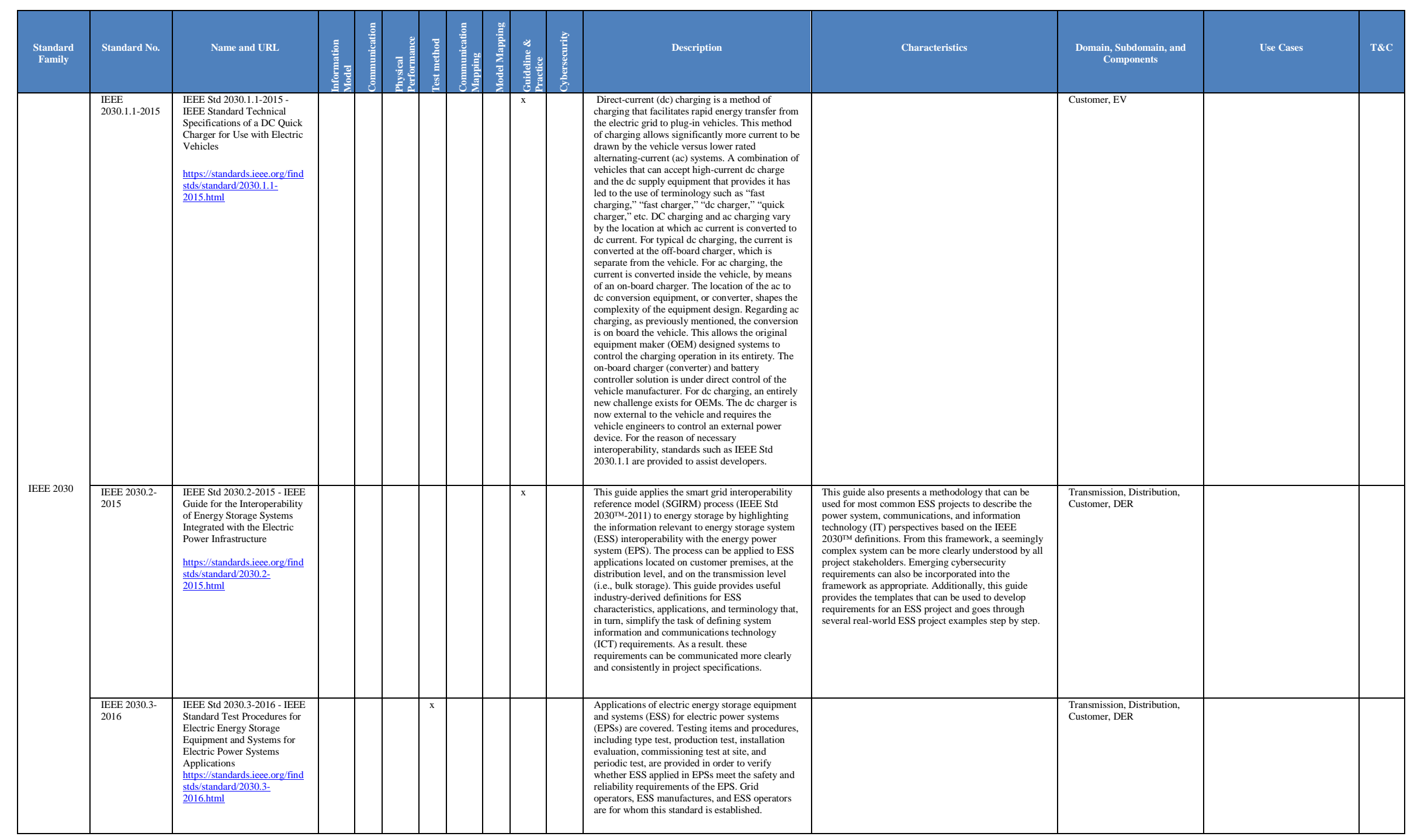




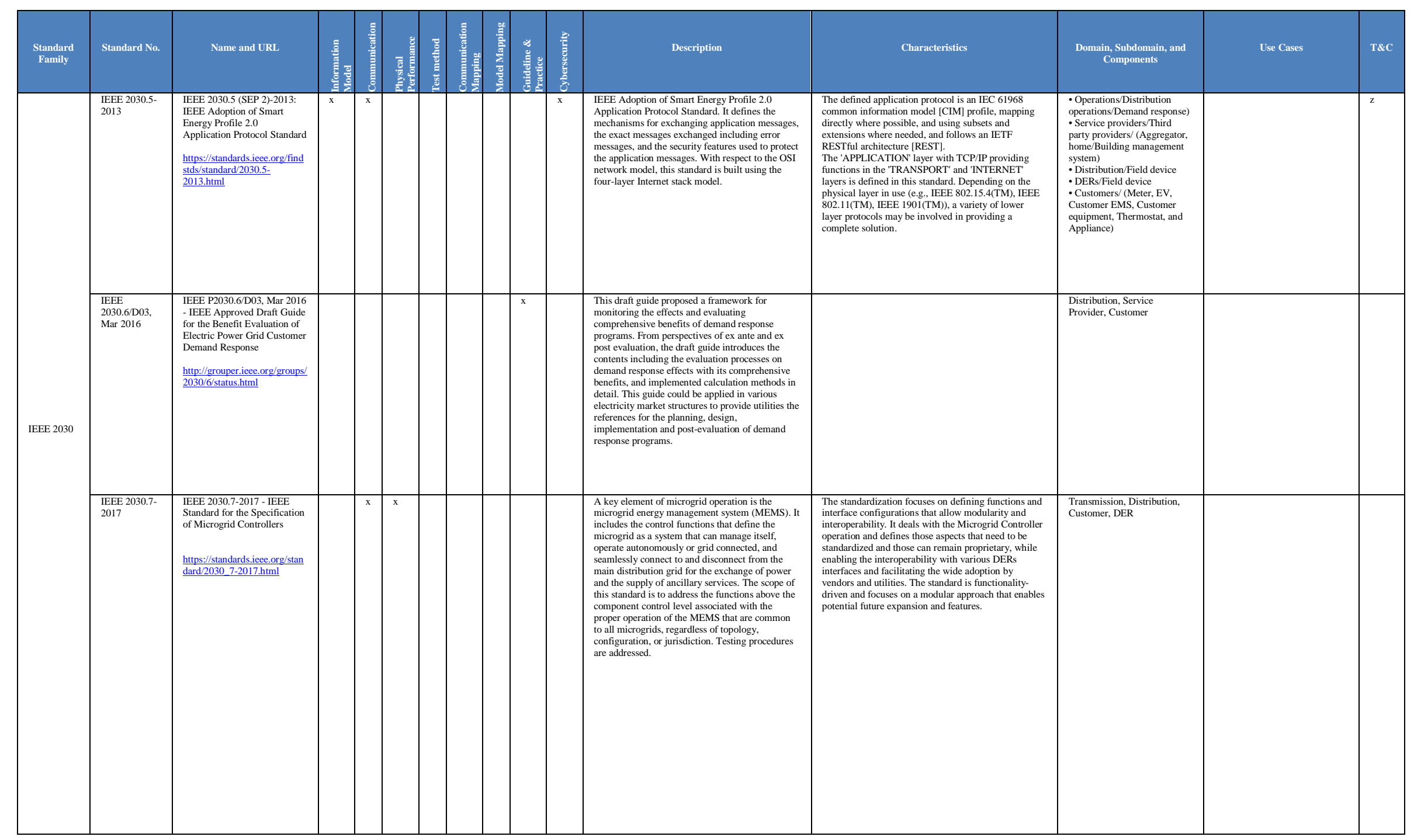




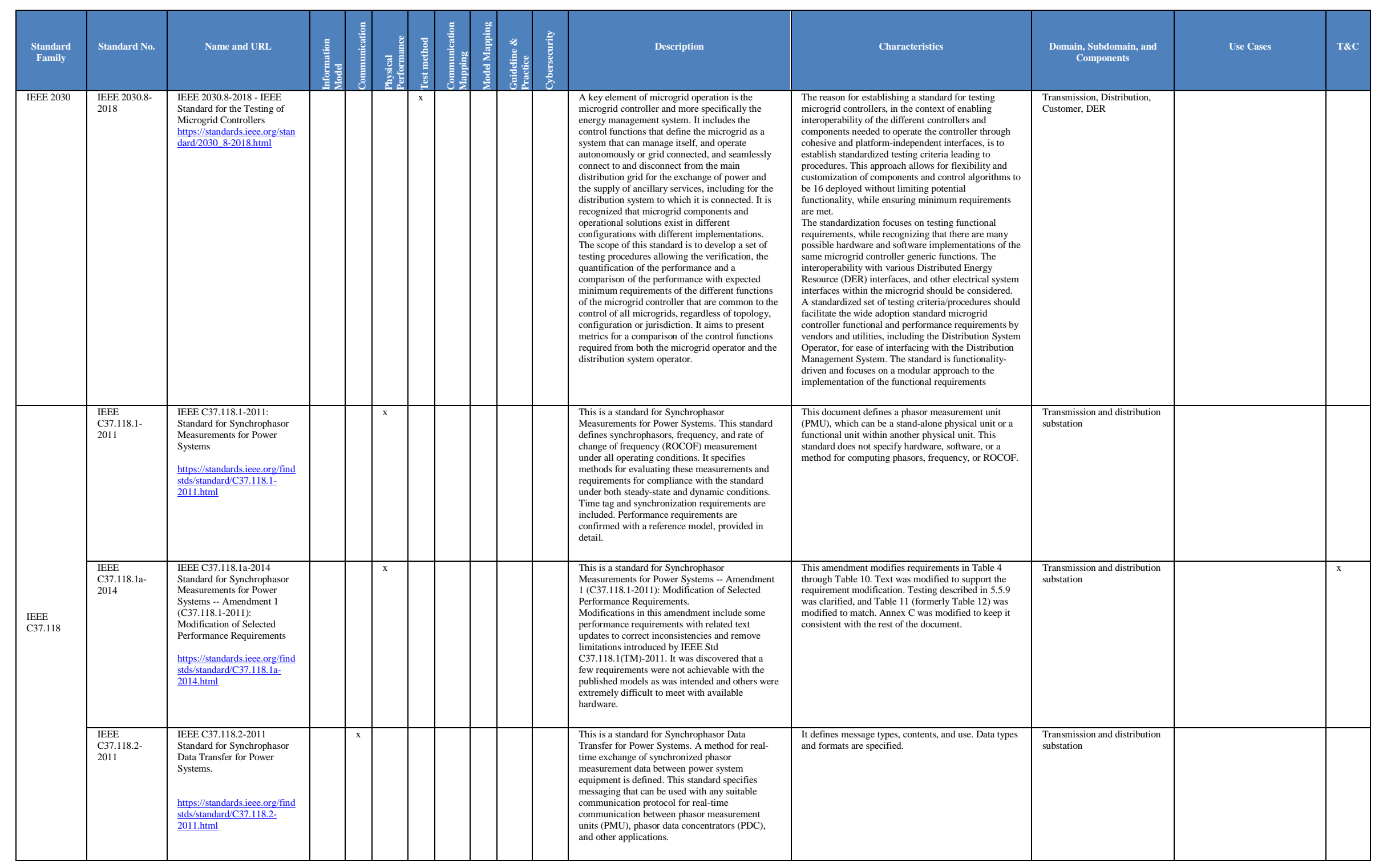




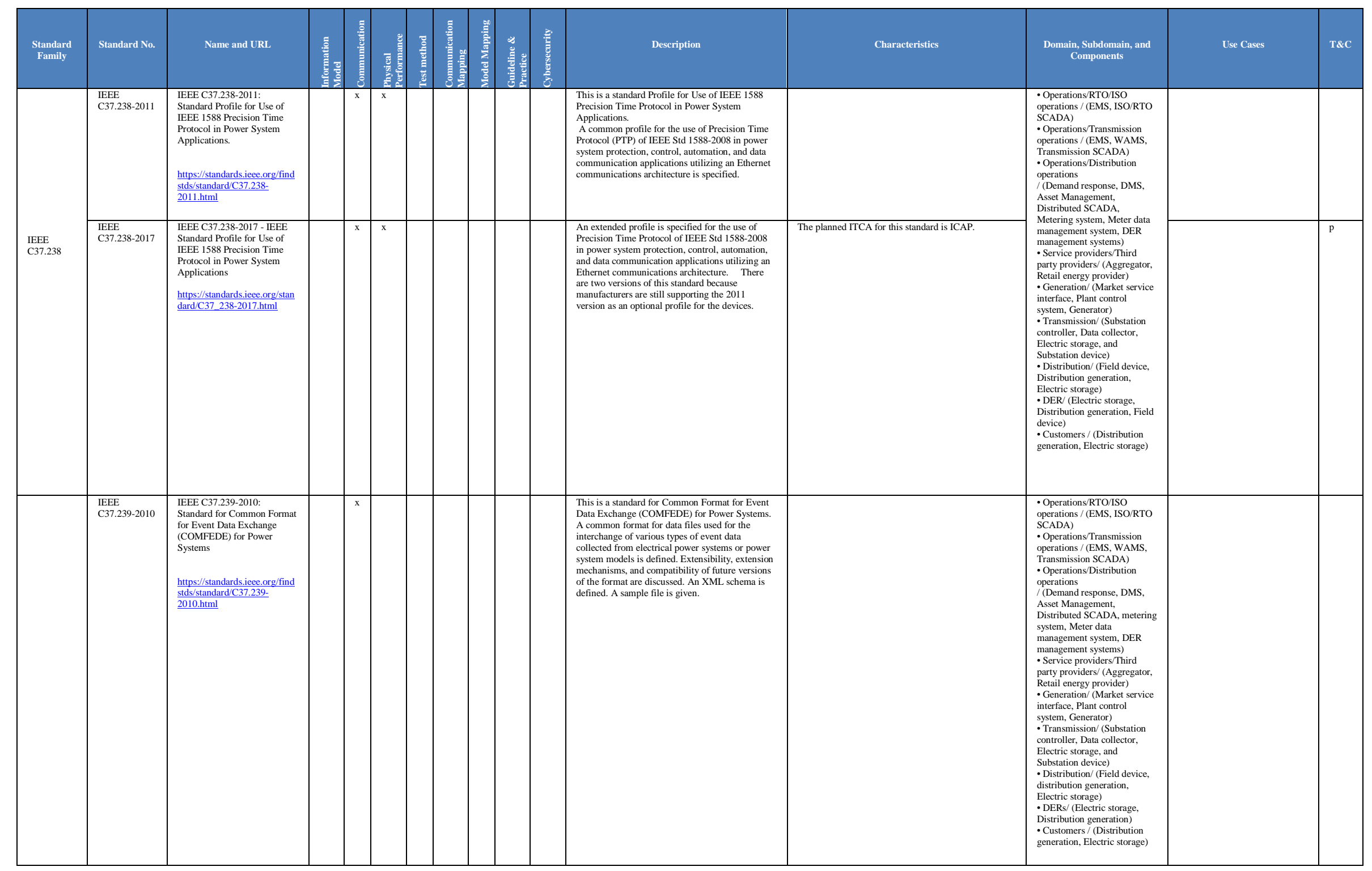




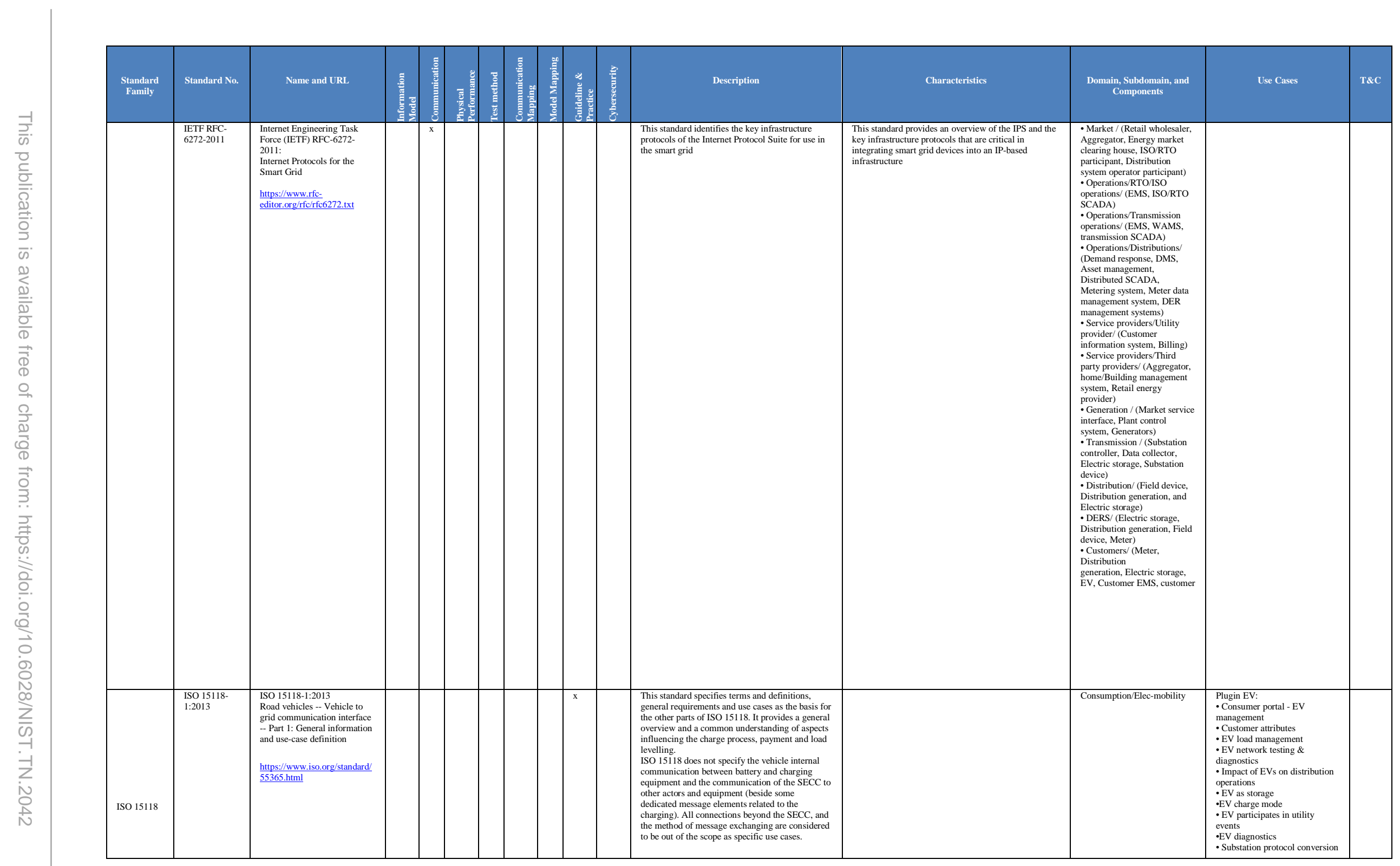




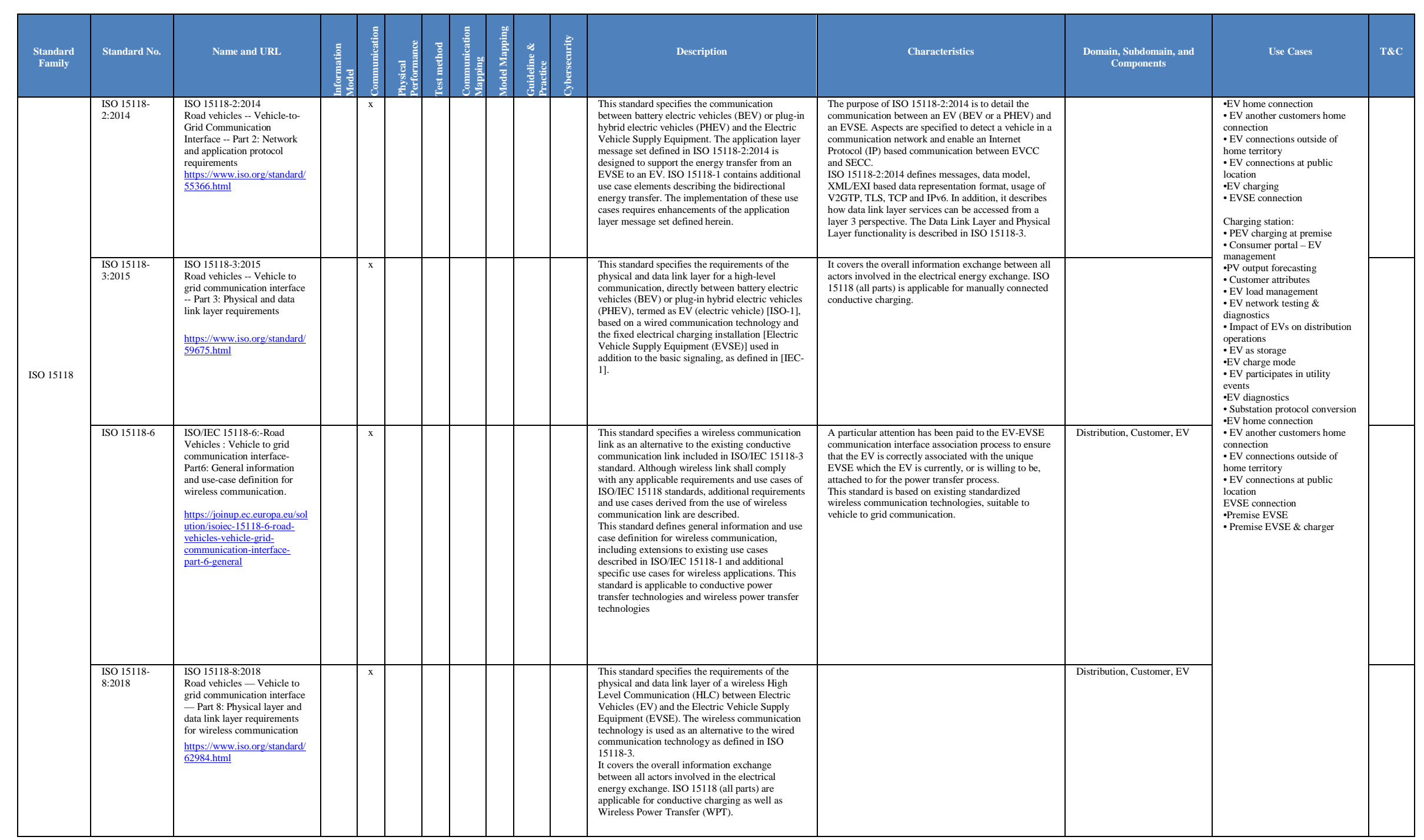




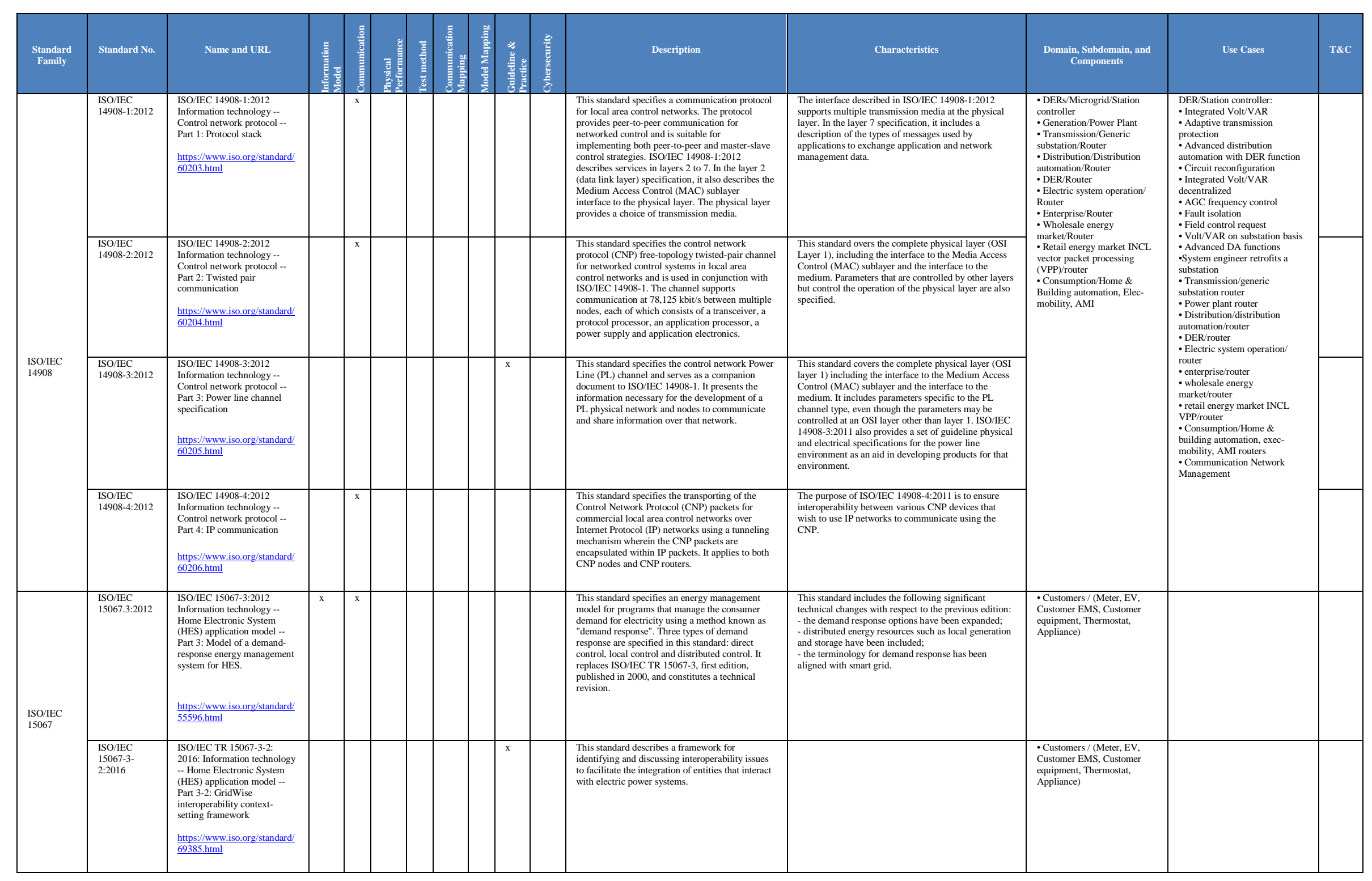




\begin{tabular}{|c|c|c|c|c|c|c|c|c|c|c|c|c|c|c|}
\hline $\begin{array}{c}\text { Standard } \\
\text { Family }\end{array}$ & Standard No. & Name and URL & & & & 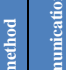 & & 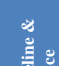 & 言 & Description & Characterisitics & $\begin{array}{l}\text { Domain, Subdomain, and } \\
\text { Components }\end{array}$ & Use Cases & T\&C \\
\hline & ITU T-G.9903 & 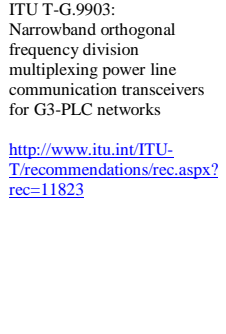 & & & & & & & & 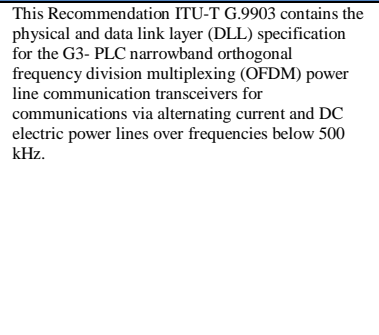 & 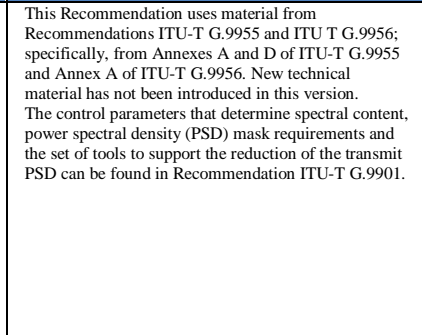 & 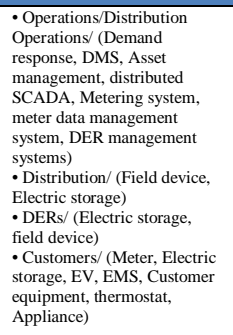 & & \\
\hline & $\begin{array}{l}\text { ITU T- } \\
\text { G.9960-2011 }\end{array}$ & 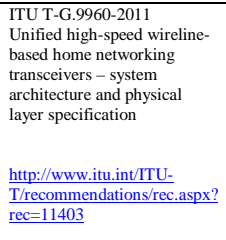 & & & & & & & & 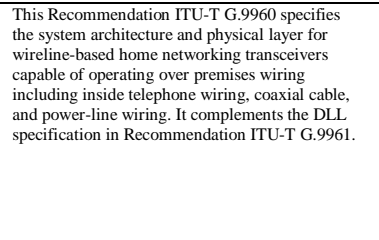 & 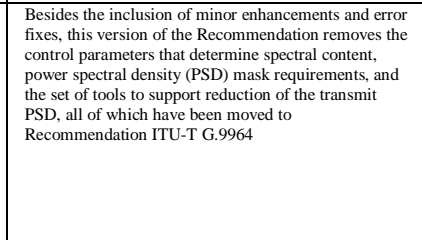 & \begin{tabular}{|l|l} 
Distribution, Customer \\
\end{tabular} & & \\
\hline & $\begin{array}{l}\text { ITU T- } \\
\text { G. }\end{array}$ & $\begin{array}{l}\text { ITU T-G.9972:2010 } \\
\text { Coexistence mechanism for } \\
\text { wirline home networking } \\
\text { transceivers } \\
\text { https://wwwwitu.intrec/T- } \\
\text { REC-G.9972-201006-I/en }\end{array}$ & & & & & & & & 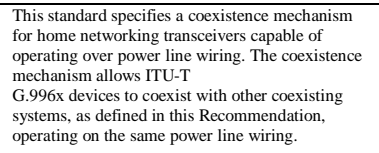 & 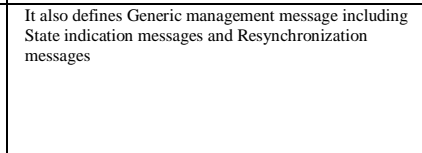 & $\begin{array}{l}\text { Distribution, Customer } \\
\end{array}$ & & \\
\hline & $\begin{array}{l}\text { Multitispeak } \\
\text { Security-V1.0 }\end{array}$ & $\begin{array}{l}\text { MultiSpeak Security-V1.0 } \\
\text { https:///www.multispeak.org/s } \\
\text { ecurity-considerations/ }\end{array}$ & & & & $\mathrm{x}$ & & & $x$ & 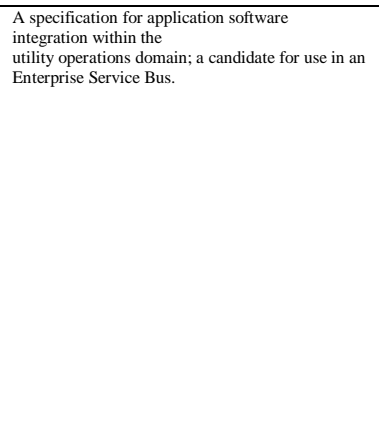 & 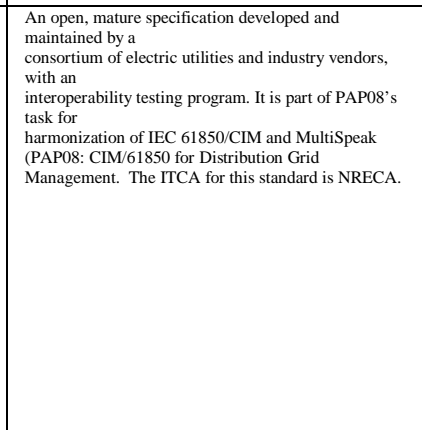 & 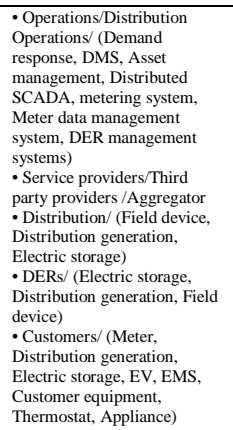 & & $\mathrm{x}$ \\
\hline
\end{tabular}




\begin{tabular}{|c|c|c|c|c|c|c|c|c|c|c|c|c|c|c|}
\hline $\begin{array}{l}\text { Standard } \\
\text { Family }\end{array}$ & Standard No. & Name and URL & 要 & 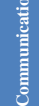 & 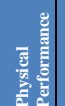 & 童 & 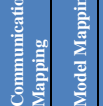 & 吾| & 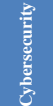 & Description & Characteristics & $\begin{array}{l}\text { Domain, Subdomain, and } \\
\text { Components }\end{array}$ & Use Cases & $\mathrm{T} \& \mathrm{C}$ \\
\hline & $\begin{array}{l}\text { MultiSpeak } \\
\text { V3.0:2015 }\end{array}$ & $\begin{array}{l}\text { MultiSpeak V3.0 } \\
\text { http://www.multispeak.org/m } \\
\text { lltispeak-specification// }\end{array}$ & $\mathrm{x}$ & $\mathrm{x}$ & & & & & & 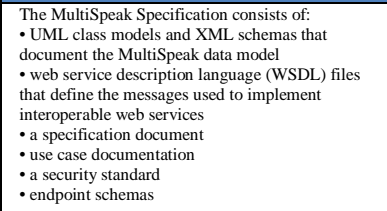 & 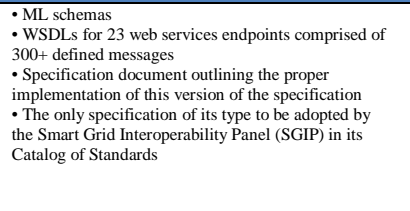 & Distribution & & $\mathrm{x}$ \\
\hline & $\begin{array}{l}\text { NAESB } \\
\text { REQ.19 }\end{array}$ & 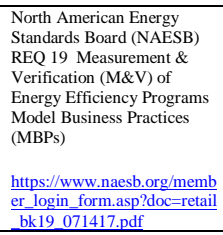 & & & & & & $\mathrm{x}$ & & \begin{tabular}{|l|} 
The standards specify two-way flows of energy \\
usage information based on a standardized \\
information model.
\end{tabular} & & $\begin{array}{l}\text { Distribution, Service } \\
\text { Provider, Customer }\end{array}$ & & \\
\hline & $\begin{array}{l}\text { NAESB } \\
\text { REQ.21 }\end{array}$ & $\begin{array}{l}\text { NAESB REQ.21 Energy } \\
\text { Services Provider Interface } \\
\text { Model Business Practices } \\
\text { (MBPS) } \\
\text { hitps:///www.naesbo.org/memb } \\
\text { er login forma.appdodoreretail } \\
\text { bk21_071417.pdf }\end{array}$ & $\mathrm{x}$ & $\mathrm{x}$ & & & & & $\mathrm{x}$ & 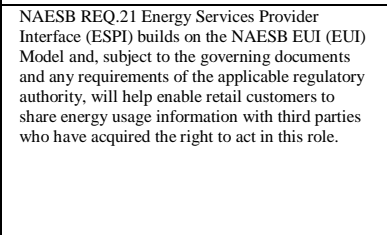 & 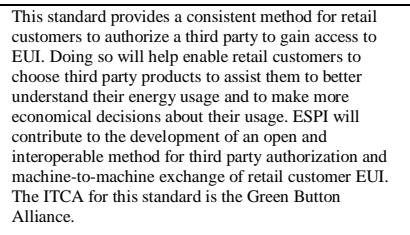 & $\begin{array}{l}\text { Distribution, Service } \\
\text { Provider, Customer }\end{array}$ & & $\mathrm{x}$ \\
\hline & $\begin{array}{l}\text { NAESB } \\
\text { REQ.22 }\end{array}$ & 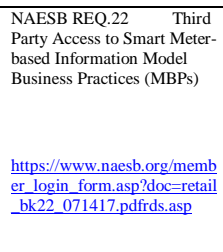 & & & & & & $\mathrm{x}$ & & 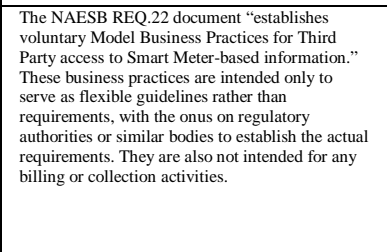 & $\begin{array}{l}\text { This standard provides guidelines for the privacy } \\
\text { business practices for Distribution Companies and } \\
\text { Third Parties when managing private } \\
\text { customer Smart Meter information. } \\
\text { ESPI applies to customer interaction systems of } \\
\text { utilities, third party servicice providerss, and customers } \\
\text { and their devices such as handheld and desktop } \\
\text { computers, thermostats, electricitity } \\
\text { meters, etc. SGIP PAP 20, Green Button EPSI } \\
\text { Evolution, is billding on this work. Additionally, open } \\
\text { source implementations for ESPI and related testing } \\
\text { tools are being developed. }\end{array}$ & $\begin{array}{l}\text { Distribution, Service } \\
\text { Provider, Customer }\end{array}$ & & \\
\hline
\end{tabular}




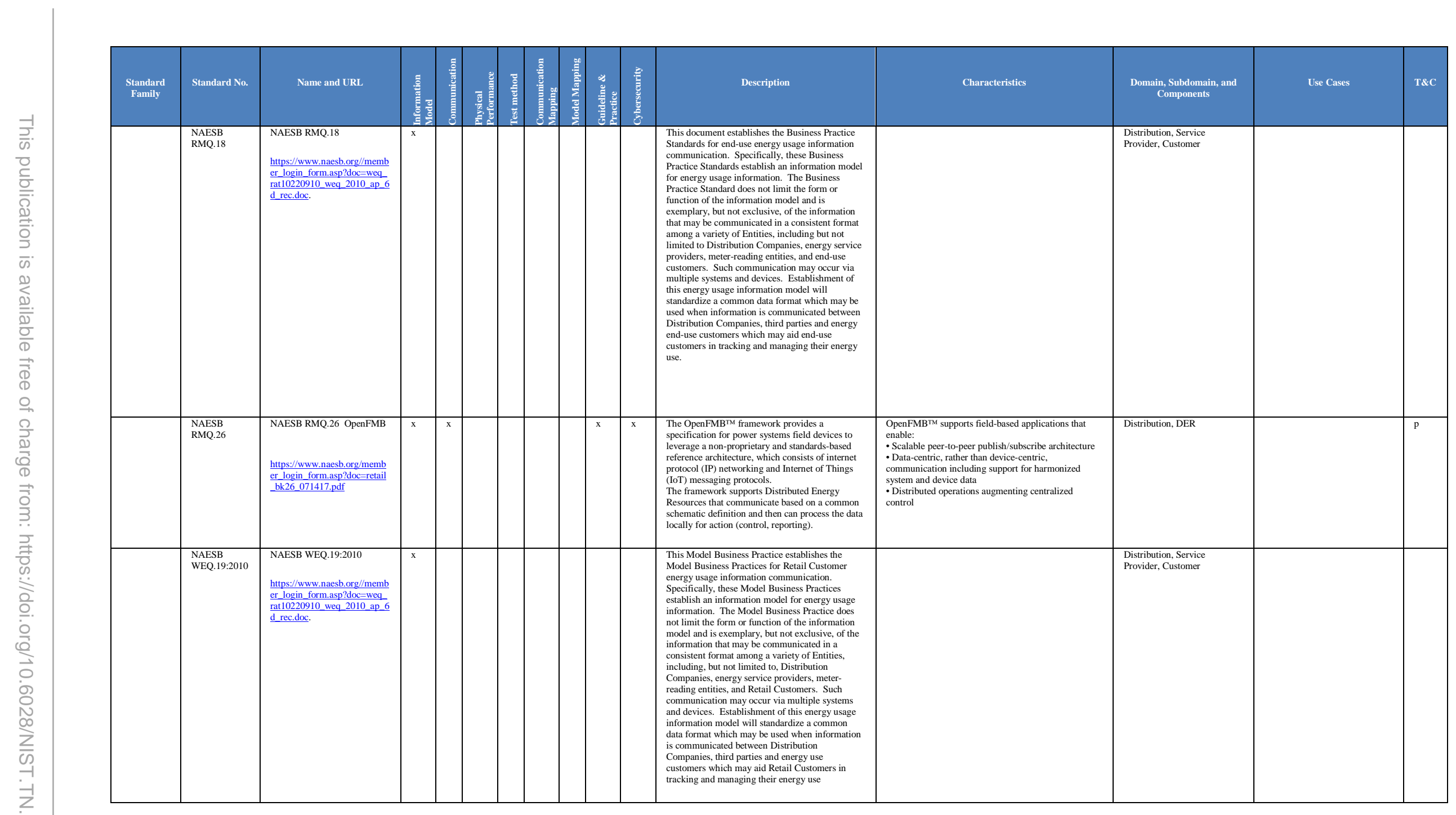




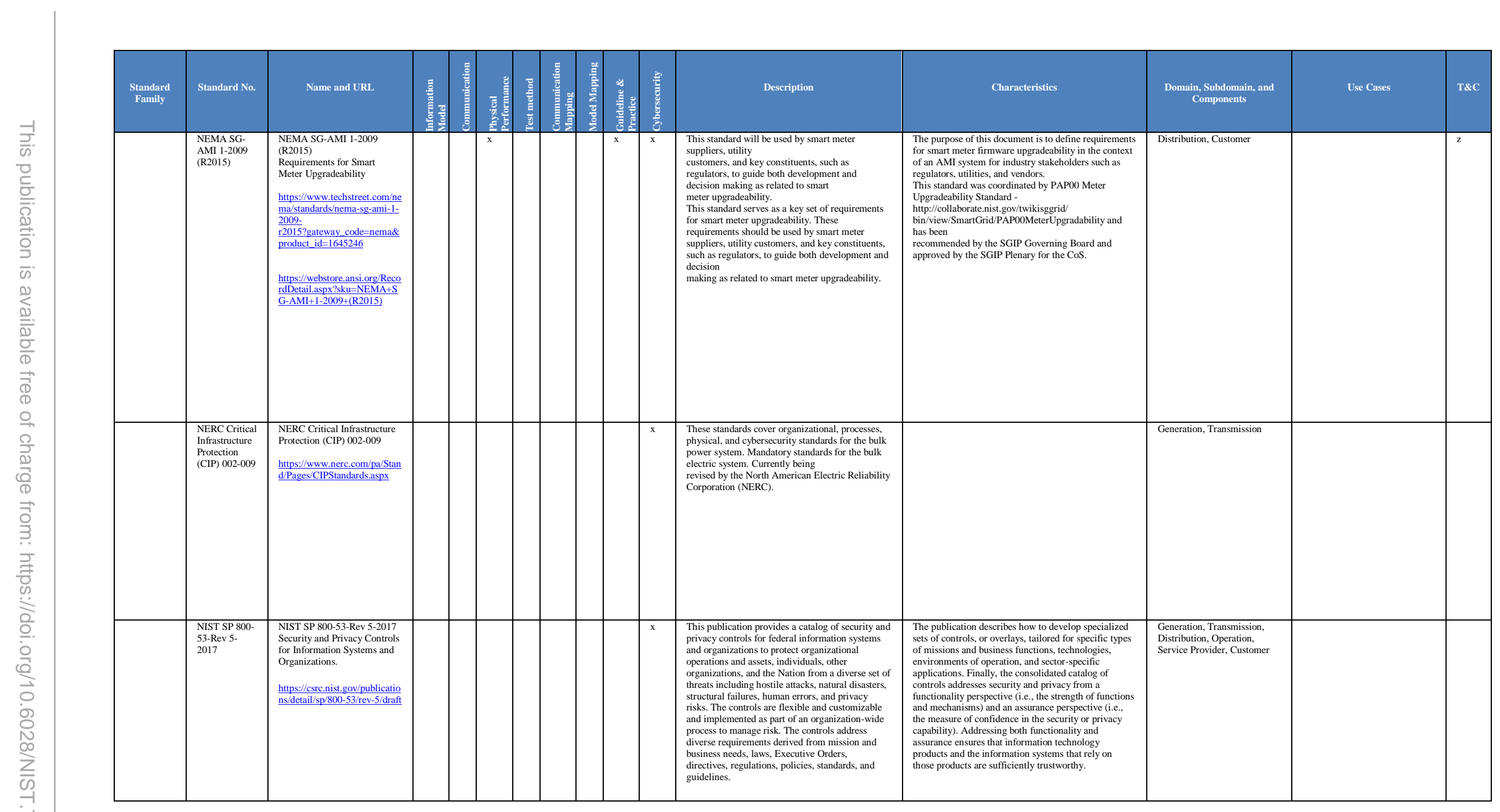




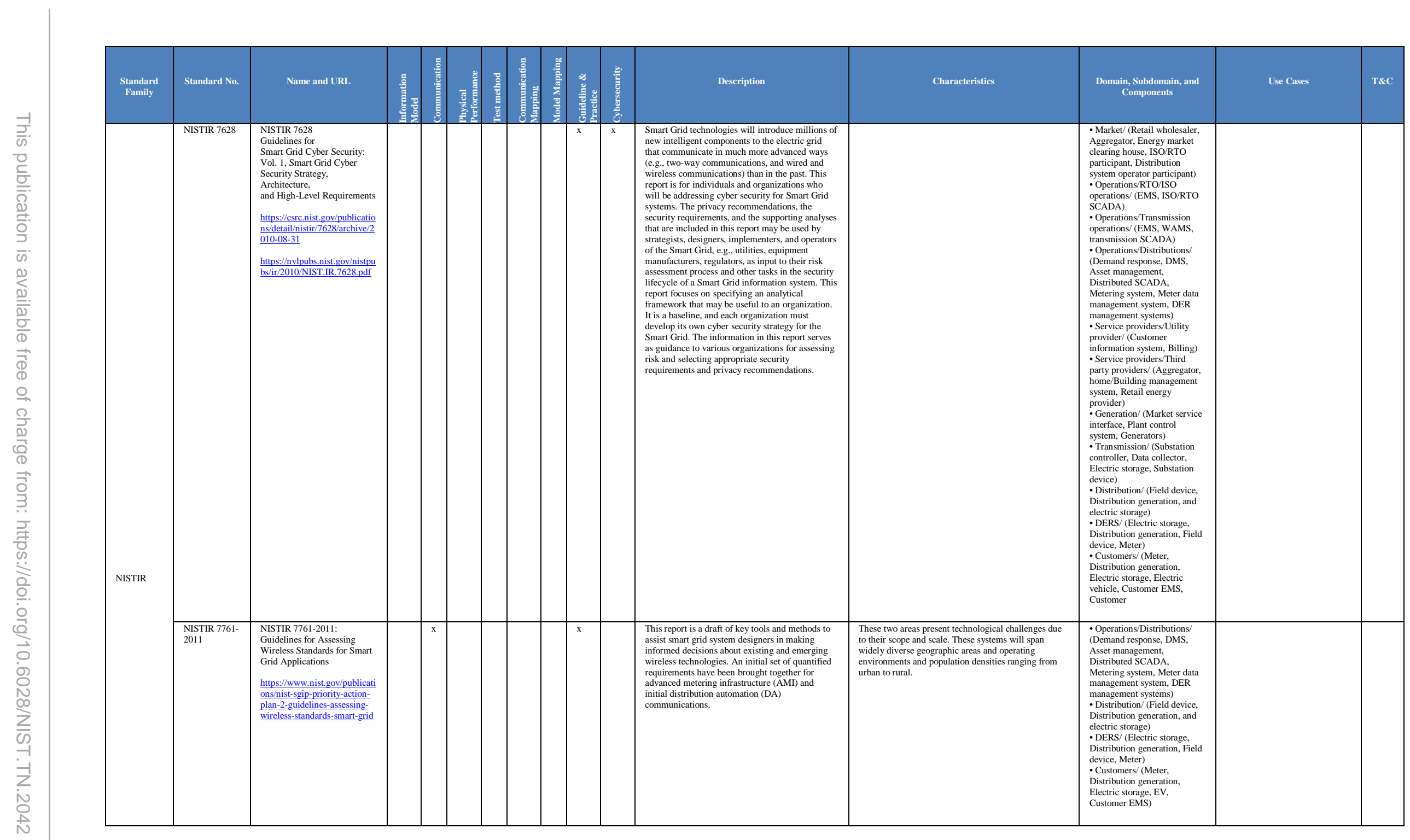




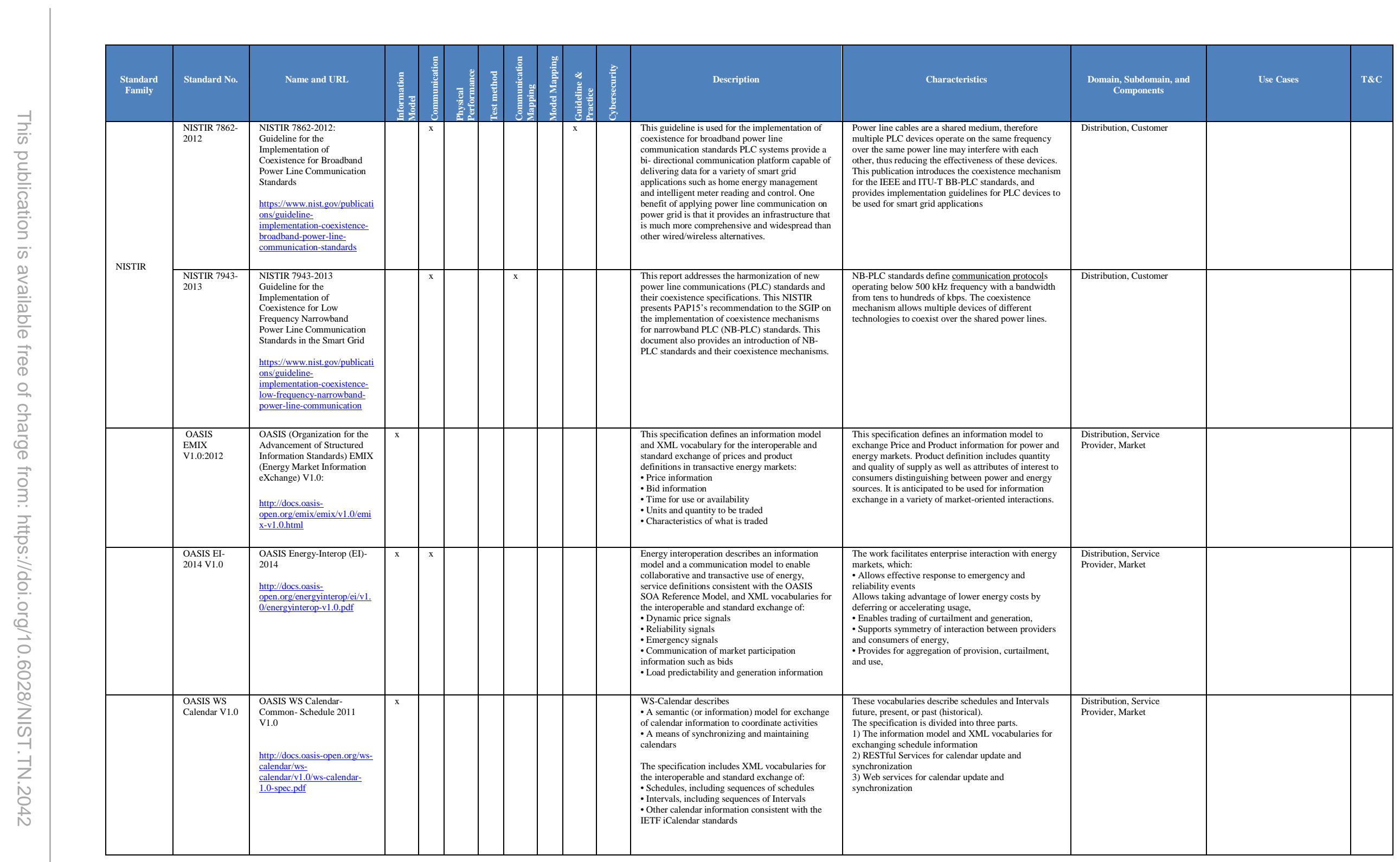




\begin{tabular}{|c|c|c|c|c|c|c|c|c|c|c|c|c|c|c|c|}
\hline $\begin{array}{c}\text { Standard } \\
\text { Family }\end{array}$ & Standard No. & Name and URL & & & & 竧 & & 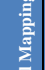 & 希 & 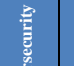 & Description & Characteristics & $\begin{array}{l}\text { Domain, Subdomain, and } \\
\text { Components }\end{array}$ & Use Cases & T\&C \\
\hline & OPC-UA & 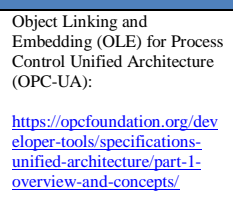 & $x$ & $\mathrm{x}$ & & & 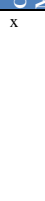 & $x$ & & & 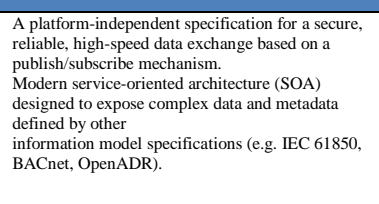 & $\begin{array}{l}\text { Works with existing binary and widely supported } \\
\text { open standard, with compliance testing program. }\end{array}$ & 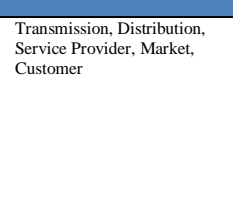 & & \\
\hline & OGC-GML & $\begin{array}{l}\text { Open Geospatial Consortium } \\
\text {-Geography Markup } \\
\text { Languaghe (OGC-GML) } \\
\text { Geography Markup Language } \\
\text { http:///www.opengeospatial.or } \\
\text { g/standards/gml }\end{array}$ & $x^{x}$ & $\mathrm{x}$ & & & & & $x$ & & 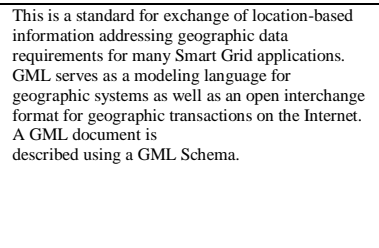 & 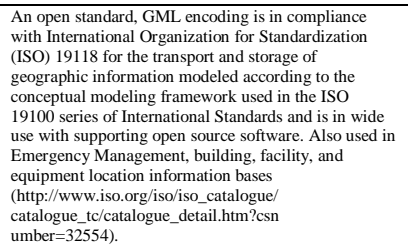 & Transmission, Distribution & & \\
\hline & OpenHAN & 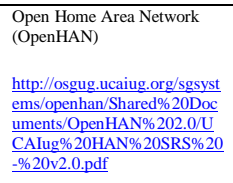 & & & & & & & $\mathrm{x}$ & & 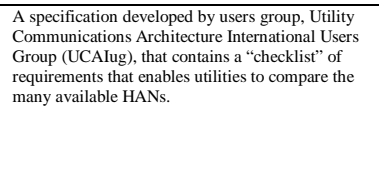 & 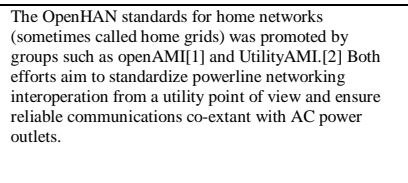 & Distribution, Customer & & \\
\hline & 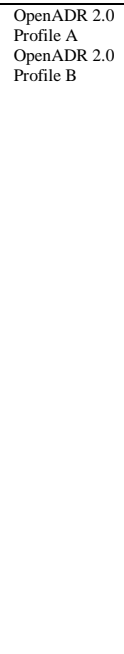 & $\begin{array}{l}\text { OpenADR 2.0 Profile A } \\
\text { OpenADR 2.0 Profile B } \\
\text { httrs://ivww.openadr.org/spec } \\
\text { ification }\end{array}$ & $x$ & $x$ & & & & & & & 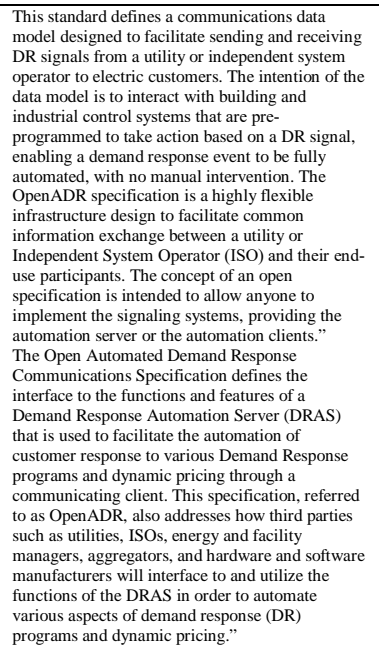 & 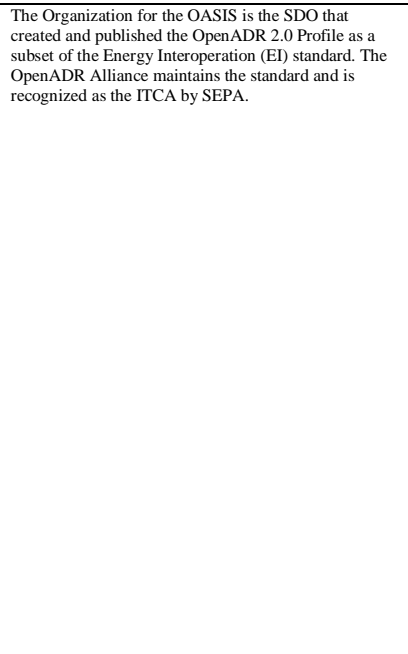 & $\begin{array}{l}\text { Distribution, Service } \\
\text { Provider, Market }\end{array}$ & & $x$ \\
\hline
\end{tabular}




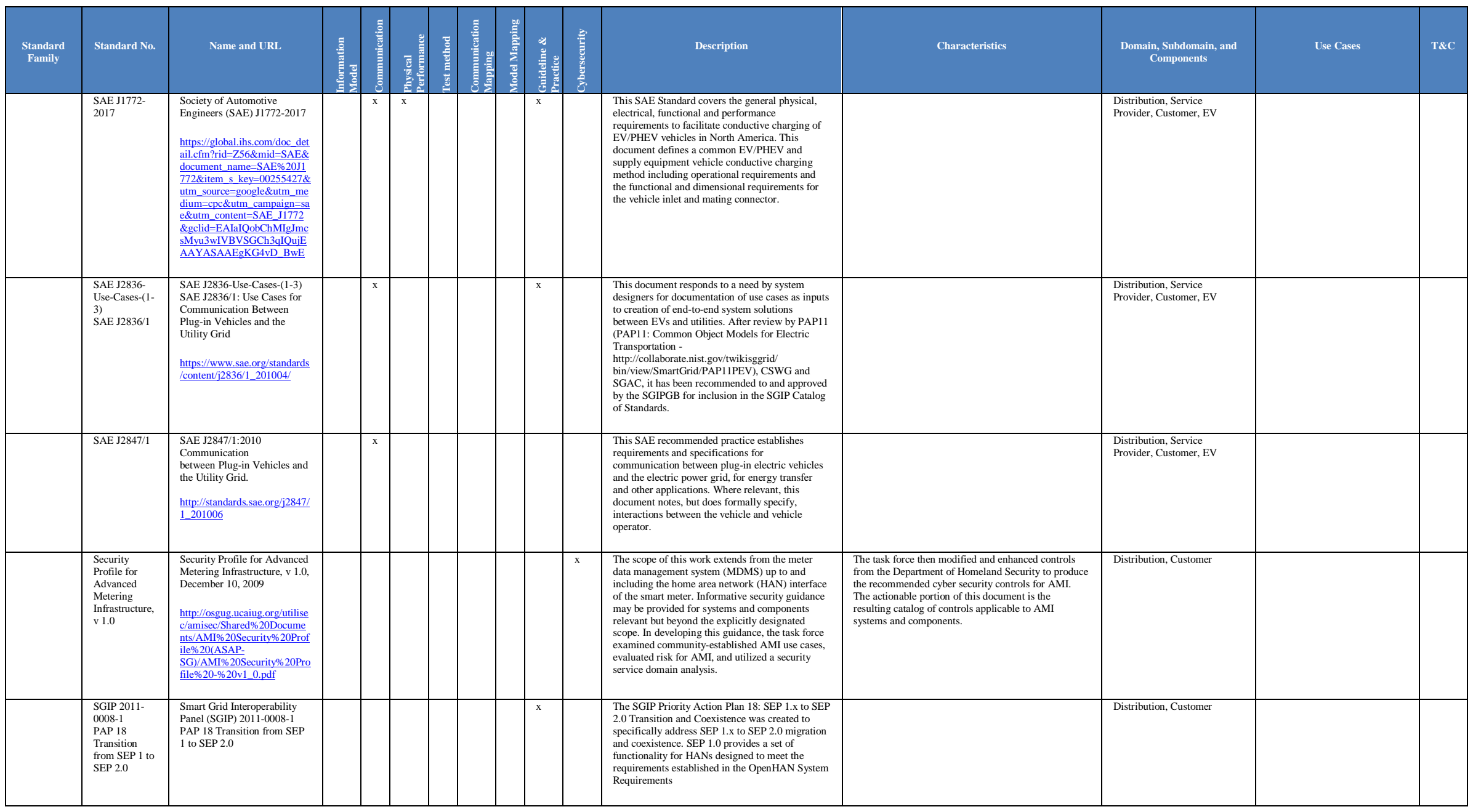

